\title{
Human hepatocyte PNPLA3 148M exacerbates rapid non-alcoholic fatty liver disease development in chimeric mice
}

Mohammad Kabbani

Rockefeller University https://orcid.org/0000-0001-9556-384X

Eleftherios Michailidis

The Rockefeller University, Laboratory of Virology and Infectious Disease https://orcid.org/0000-00029907-4346

\section{Sandra Steensels}

Weill Cornell Medicine

Clifton Fulmer

The Cleveland Clinic

Joseph Luna

The Rockefeller University

Jérémie Le Pen

The Rockefeller University

Matteo Tardelli

Weill Cornell Medicine

Brandon Razooky

The Rockefeller University

Inna Ricardo-Lax

The Rockefeller University

Chenhui Zou

The Rockefeller University

Briana Zeck

NYU Langone

Ansgar Stenzel

The Rockefeller University

Corrine Quirk

The Rockefeller University

Lander Foquet

Yecuris Corporation

Alison Ashbrook

The Rockefeller University 


\section{William Schneider}

The Rockefeller University

\section{Serkan Belkaya}

The Rockefeller University

\section{Gadi Lalazar}

The Rockefeller University

\section{Yupu Liang}

The Rockefeller University

\section{Meredith Pittman}

Weill Cornell Medicine

\section{Lindsey Devisscher}

Ghent University

\section{Hiroshi Suemizu}

Central Institute for Experimental Animals

\section{Neil Theise}

NYU Langone

\section{Luis Chiriboga}

NYU Langone

\section{David Cohen}

Division of Gastroenterology \& Hepatology, Weill Cornell Medical College, New York, NY 10021

\section{Robert Copenhaver}

Yecuris Corporation

\section{Markus Grompe}

Yecuris (United States)

\section{Philip Meuleman}

Ghent University

\section{Baran Ersoy}

Weill Cornell Medicine

\section{Charles Rice}

The Rockefeller University

Ype de Jong ( $\sim$ ydj2001@med.cornell.edu )

Weill Cornell Medicine https://orcid.org/0000-0001-6357-2370

\section{Article}

Keywords: advanced non-alcoholic fatty liver disease (NAFLD), patatin-like phospholipase domaincontaining protein 3 (PNPLA3-I148M),

Posted Date: February 10th, 2021 
DOl: https://doi.org/10.21203/rs.3.rs-156250/v1

License: (c) (1) This work is licensed under a Creative Commons Attribution 4.0 International License. Read Full License 
1 Human hepatocyte PNPLA3 148M exacerbates rapid non-alcoholic fatty liver disease

2 development in chimeric mice

3

4 Mohammad Kabbani $^{1,2, \star}$, Eleftherios Michailidis ${ }^{1, \star}$, Sandra Steensels $^{3}$, Clifton G. Fulmer ${ }^{4,5}$,

5 Joseph M. Luna ${ }^{1}$, Jérémie Le Pen ${ }^{1}$, Matteo Tardelli3 ${ }^{3}$, Brandon Razooky ${ }^{1}$, Inna Ricardo-Lax ${ }^{1}$,

6 Chenhui Zou ${ }^{1,3}$, Briana Zeck ${ }^{6}$, Ansgar F. Stenzel ${ }^{1,7}$, Corrine Quirk ${ }^{1}$, Lander Foquet ${ }^{8}$, Alison W.

7 Ashbrook ${ }^{1}$, William M. Schneider ${ }^{1}$, Serkan Belkaya ${ }^{9}$, Gadi Lalazar ${ }^{3,10}$, Yupu Liang ${ }^{11}$, Meredith

8 Pittman $^{4}$, Lindsey Devisscher ${ }^{12}$, Hiroshi Suemizu ${ }^{13}$, Neil D. Theise ${ }^{6}$, Luis Chiriboga ${ }^{6}$, David E.

9 Cohen $^{3}$, Robert Copenhaver ${ }^{8}$, Markus Grompe 8,14 , Philip Meuleman ${ }^{15}$, Baran A. Ersoy ${ }^{3}$, Charles

10 M. Rice ${ }^{1}$ and Ype P. de Jong ${ }^{1,3, \# .}$

11

12 1. Laboratory of Virology and Infectious Disease, The Rockefeller University, New York, $13 \quad$ NY10065.

14 2. Department of Gastroenterology, Hepatology and Endocrinology, Hannover Medical School, 15 Hannover, Germany.

16 3. Division of Gastroenterology and Hepatology, Weill Cornell Medicine, New York, NY10065.

17 4. Department of Pathology, Weill Cornell Medicine, New York, NY10065.

18 5. Robert J. Tomsich Pathology and Laboratory Medicine Institute, The Cleveland Clinic, $19 \quad$ Cleveland, Ohio, $\mathrm{OH} 44195$.

20 6. Department of Pathology, NYU Langone, New York, NY10028.

21 7. Department of Infectious Diseases, Molecular Virology, Heidelberg University, Heidelberg, Germany.

23 8. Yecuris Corporation, Tualatin, OR97062.

24 9. St. Giles laboratory of Human Genetics of Infectious Diseases, The Rockefeller University, $25 \quad$ New York, NY10065. 
26 10. Laboratory of Cellular Biophysics, The Rockefeller University, New York, NY10065.

27 11.Center for Clinical and Translational Science, The Rockefeller University, New York, $28 \quad$ NY10065.

29 12. Department of Basic and Applied Medical Sciences, Gut-Liver Immunopharmacology Unit, 30 Ghent University, Ghent, Belgium

31 13. Central Institute for Experimental Animals, Kanagawa, Japan.

32 14. Department of Pediatrics, Oregon Stem Cell Center, Oregon Health and Science University, 33 Portland, OR97239.

34 15. Laboratory of Liver Infectious Diseases, Ghent University, Ghent, Belgium.

$35{ }^{*}$ These authors contributed equally.

36 \# Lead contact.

37

38 Correspondence: Ype de Jong, Division of Gastroenterology and Hepatology, Weill Cornell 39 Medicine, 1305 York Ave, New York, NY 10065, Tel: 646-962-4000, Fax: 646-962-0355, Email: 40 ydj2001@med.cornell.edu.

41

42 Short title: Hepatocyte PNPLA3-148M worsens steatohepatitis 


\section{SUMmARY}

44 Advanced non-alcoholic fatty liver disease (NAFLD) is a rapidly emerging global health problem

45 associated with pre-disposing genetic polymorphisms, most strikingly an isoleucine to methionine 46 substitution in patatin-like phospholipase domain-containing protein 3 (PNPLA3-I148M). Here, we 47 study how human hepatocytes with PNPLA3 148I and 148M variants engrafted in the livers of 48 chimeric mice respond to hypercaloric diets. As early as 4 weeks, mice developed dyslipidemia, 49 impaired glucose tolerance, and steatohepatitis selectively in the human graft, followed by 50 pericellular fibrosis after 8 weeks of hypercaloric feeding. The PNPLA3 148M variant, either from 51 a homozygous 148M human donor or overexpressed in a homozygous 148I donor background, 52 caused microvesicular and more severe steatosis. In these livers hepatocytes displayed frequent 53 ballooning degeneration, resulting in more active steatohepatitis than in 148I livers. We conclude 54 that PNPLA3 148M in human hepatocytes exacerbates NAFLD. These models will facilitate 55 mechanistic studies into human genetics associated with advanced fatty liver diseases. 


\section{INTRODUCTION}

Non-alcoholic fatty liver disease (NAFLD) has become the most prevalent liver disease in many countries, affecting approximately $25 \%$ of the world population. ${ }^{1}$ NAFLD is associated with obesity, insulin resistance, dyslipidemia and hypertension. ${ }^{2}$ A subset of patients with NAFLD develop non-alcoholic steatohepatitis (NASH), which is widely believed to drive fibrosis progression towards advanced liver disease including cirrhosis and hepatocellular carcinoma $(\mathrm{HCC}) .^{3}$

The complex nutritional and environmental factors leading to NAFLD remain to be better defined. ${ }^{4}$ In addition, a number of genetic variants have been associated with advanced NAFLD. ${ }^{5}$ The first genetic polymorphism identified in a population with increased hepatic steatosis was rs738409 in patatin-like phospholipase domain containing protein-3 (PNPLA3). ${ }^{6}$ This polymorphism encodes for an isoleucine to methionine substitution at codon position 148 in PNPLA3. Since its identification by Hobbs and colleagues, the PNPLA3-I148M variant has been globally associated with advanced NAFLD and other liver diseases including HCC. ${ }^{7}$

The mechanisms by which PNPLA3-148M drives advanced NAFLD remains an active area of investigation.8, 9 Although PNPLA3 is expressed in hepatocytes, hepatic stellate cells and adipocytes, accumulating evidence from mouse models indicates that hepatocyte-specific PNPLA3-148M promotes the pathogenesis of NAFLD. Transgenic overexpression of human PNPLA3-148M in mouse hepatocytes but not adipocytes exacerbated hepatic steatosis. ${ }^{10}$ And while increased hepatic fat content was observed in Pnpla3-148M knock-in mice, ${ }^{11}$ this was reversed by silencing Pnpla3 in hepatocytes. ${ }^{12}$ Functionally, PNPLA3 exhibits hydrolase activity towards triglycerides and acyltransferase activity for polyunsaturated fatty acids in phospholipids. ${ }^{8}$ PNPLA3-148M differs from 148I in several ways: PNPLA3-148M was shown to have decreased hydrolase activity against triglycerides, ${ }^{13}$ increased acyltransferase activity for lysophosphatidic acid, ${ }^{14}$ resistance to ubiquitylation and proteasomal degradation, ${ }^{15}$ and inhibition of adipose 
81 triglyceride lipase on lipid droplets via interaction with $\alpha / \beta$-hydrolase domain containing $5 .{ }^{16,17}$

82 How these and other mechanistic differences drive NAFLD progression remains to be further 83 elucidated. $\mathrm{NASH}$ and sustained weight loss remains the only proven intervention. Given its complex pathogenesis, many different pathways are under pre-clinical and clinical investigation, some of which have resulted in compounds that progressed to Phase 3 trials. ${ }^{18}$ Several of these pathways were identified in murine NASH models that share limited features with human disease. ${ }^{19}$ Furthermore, investigational strategies that target subsets of NASH patients, e.g. those with genetic variants, are further complicated by species differences. In the case of PNPLA3, there is low sequence homology between the human and mouse orthologues. The human protein is 97 amino acids longer than the mouse PNPLA3. The relative mRNA expression starkly differs between adipose tissue and liver in each species. ${ }^{20,21}$ and it remains unclear if the same transcription factors regulate its expression in murine and human hepatocytes. ${ }^{22,23}$ Elucidation of the precise mechanisms by which human genetic variants in hepatocytes affect steatohepatitis would be aided by minimizing species-specific variances in a physiologically relevant experimental setting.

Here, we established a human hepatocyte NAFLD model. We applied this model to study human hepatocyte PNPLA3-148M, either from a 148M homozygous human hepatocyte donor and by overexpressing PNPLA3 variants in a 148I homozygous donor background. Our results show that human hepatocytes swiftly develop steatohepatitis in response to hypercaloric feeding and that NAFLD activity is higher when hepatocytes express PNPLA3-148M. These models will 
RESULTS

Human hepatocytes in chimeric mice on Western diet rapidly develop steatosis

To investigate how the human graft in liver chimeric mouse models responds to a 109 hypercaloric diet, we first created humanized mice by transplanting primary human hepatocytes $110(\mathrm{PHH})$ into preconditioned immunocompromised Fah ${ }^{-/}$NOD Rag1/- $/ 12$ rgull $^{\text {naNRG}}$ (F) mice as 111 described previously ${ }^{24,25}$. Cycling the protective drug nitisinone ${ }^{26}$ resulted in at least $50 \%$ 112 humanization after which mice were subjected to an ad libitum Western-style diet (WD), which 113 consists of $60 \%$ fat in diet and $10 \%$ sucrose in drinking water (Fig 1a). WD feeding did not result 114 in graft loss as determined by persistently high ${ }^{27-29}$ human serum albumin (hAlb) levels (Suppl 115 Fig S1a). Periodic examination of huFNRG mouse livers showed steatosis ${ }^{30}$ as early as 4 weeks 116 on WD as evidenced by hematoxylin and eosin (H\&E) staining (Fig 1b) and Oil Red O staining 117 (Suppl Fig S1b). Steatosis almost exclusively affected the human graft whereas the remaining mouse hepatocytes, which appear larger with darker cytoplasm after H\&E staining, rarely developed steatosis. The contrast in steatosis between mouse and human hepatocytes was 120 further evident by using a human-specific marker (Fig 1c). Livers from highly humanized mice 121 with severe steatosis showed sparing around periportal regions (Fig $\mathbf{1 b}$ ), reminiscent of zonal 122 steatosis in adult NAFLD patients. The zonal predominance was further highlighted by glutamine synthetase staining, which is mostly expressed in zone 3 hepatocytes (Fig 1d). In order to quantify steatosis in huFNRG mice, the human areas of chimeric livers were scored blindly according to clinical criteria. ${ }^{30}$ All animals developed mild to severe steatosis as early as 4 weeks on WD. Steatosis persisted for 20 weeks on WD, which contrasted with huFNRG mice on control chow

127 (chow) that never developed more than 5\% steatosis (Fig 1e). Clinical scoring was confirmed by total triacylglycerol (TAG) quantification in huFNRG livers, which was 7 to 11 -fold higher in WD 129 than chow mice (Fig 1f), and liver total cholesterol (Suppl Fig S1c). Overall, the accumulation of 130 TAG in huFNRG livers correlated to the steatosis score of the human graft (Suppl Fig S1d). 
131 These findings show that human hepatocytes in huFNRG mice rapidly developed moderate 132 steatosis in response to WD feeding.

We next tested if the marked steatosis difference between human and mouse hepatocytes

134 was due to the NOD mouse background, the intermittent nitisinone used to protect Fah ${ }^{-/-}$mice 135 from liver injury ${ }^{31}$, or the hepatocyte transplantation biology of the FNRG model. First, we 136 subjected immunodeficient NOD Rag $1^{--} \| 2 r^{\text {null }}(\mathrm{NRG})$ mice to WD feeding and nitisinone cycling.

137 Even after 12 weeks on WD NRG livers developed minimal steatosis (Suppl Fig S1e), consistent with previous reports that $T$ cell deficient mice are protected from experimental NAFLD. ${ }^{32-34}$ To test if FNRG liver injury and/or hepatocyte repopulation physiology made hepatocytes susceptible 140 to hypercaloric feeding we transplanted NRG mouse hepatocytes into FNRG mice. These animals 141 were designated 'murinized' FNRG (muFNRG) mice. After 4 weeks on WD muFNRG livers did 142 not display steatosis, but small areas of hepatocyte steatosis were present after 12 weeks of WD feeding (Fig 1g) which was in line with liver TAG levels (Suppl Fig S1f). These results show that human hepatocytes responded differently to WD feeding than mouse hepatocytes in these 145 models.

We then tested if rapid steatosis was specific to this human hepatocyte donor (PHH1) and to the FNRG liver injury model, extending our protocols to other donors and liver chimeric mouse models. We first transplanted PHH2 into FNRG mice and thymidine kinase transgenic (TK-NOG) 149 liver injury mice. ${ }^{35}$ Despite reaching lower levels of humanization in both models, human 150 hepatocyte islands developed steatosis in response to WD feeding but not on chow (Suppl Fig 151 S1g). We also transplanted a third $\mathrm{PHH}$ donor (PHH3) into urokinase plasminogen activator 152 (uPA)/SCID mice ${ }^{36}$ and FNRG mice. Interestingly, humanized (hu-)uPA/SCID mice showed mild steatosis in the human graft on chow, which contrasted to the absence of steatosis in huFNRG

154 livers on chow (Suppl Fig S1h). In the huFNRG model, PHH3 hepatocytes developed steatosis 155 after 4 weeks of WD feeding. These data illustrate that humanized livers rapidly became steatotic 
156 following WD feeding regardless of the $\mathrm{PHH}$ donor, with different requirements for hypercaloric

157 feeding between liver injury models.

Combined, these results demonstrate that human hepatocytes but not mouse hepatocytes

159 in liver chimeric mice developed moderate steatosis as early as 4 weeks on WD.

161 Western diet affects systemic metabolic parameters of chimeric FNRG mice

NAFLD clinically overlaps with obesity, dyslipidemia, insulin resistance and possibly

163 hypertension. ${ }^{2}$ To determine whether WD affects metabolic parameters in liver chimeras, we first 164 measured body weight accumulation in adult huFNRG mice on an ad libitum WD or control chow 165 (chow) diet. Compared to chow, WD caused nearly $20 \%$ increase in body weight within 4 weeks 166 on the diet and maintained this difference through 12 weeks on WD (Fig 2a). Furthermore, the 167 gonadal fat fraction (Fig 2b) increased 2- to 2.4-fold in huFNRG mice on WD compared to chow.

168 These data demonstrate that WD feeding resulted in weight gain and gonadal fat accumulation 169 in huFNRG mice.

We then tested plasma lipids in chimeric mice. Hepatocytes are central to lipid homeostasis, and humanized mice have plasma cholesterol profiles shifted toward lower density

172 lipoproteins compared to wild type mice. ${ }^{37}$ Similar to previous reports, ${ }^{38-40}$ huFNRG on chow contained more LDL than HDL cholesterol particles compared to muFNRG mice (Fig 2c). Four weeks of WD feeding resulted in stark increases in VLDL and LDL cholesterol in huFNRG plasma.

175 Notably, the HDL fraction in huFNRG mice decreased with WD feeding whereas it rose in WD-

176 fed muFNRG mice compared to chow (Suppl Fig S2a). Relative to chow, WD increased fasting 177 serum triglycerides fractions, mostly in VLDL, and to a lesser extent in LDL fractions in huFNRG 178 mice (Fig 2d, Suppl Fig S2b). These data show that WD feeding of huFNRG mice increased 179 lower density lipoproteins and decreased HDL. 
Given the strong clinical overlap between NAFLD and type 2 diabetes, we tested the effect

181 of WD on glucose tolerance in huFNRG mice relative to muFNRG animals. While humanized 182 animals started at a higher baseline, 4 weeks on WD resulted in a 2.2-fold increase in fasting 183 blood glucose both in huFNRG and muFNRG mice (Fig 2e). Fasting mouse insulin levels on 184 chow appeared higher in humanized than murinized FNRG animals, and increased only slightly 185 in huFNRG animals after 4 weeks on WD (Fig 2f). To further characterize glucose metabolism, 186 fasting animals were subjected to glucose, insulin or pyruvate tolerance tests. After 4 weeks on 187 WD, huFNRG mice displayed severely impaired glucose clearance with 3.1-fold higher 188 cumulative blood glucose than chow, whereas this difference was 1.67 -fold higher in muFNRG 189 mice (Fig 2g). By contrast huFNRG mice on WD remained responsive to insulin challenge (Fig 190 2g). To assess gluconeogenesis in these animals, a pyruvate injection after fasting for 16 hours 191 resulted in a sharper rise in blood glucose in huFNRG on a WD than chow (Fig 2g). Eight weeks 192 of WD feeding further prolonged time for glucose clearance in huFNRG mice (Suppl Fig S2c) 193 without affecting endogenous insulin secretion (Suppl Fig S2d). These data show that WD 194 feeding of chimeric FNRG mice affected glucose homeostasis without altering insulin-mediated 195 glucose uptake.

Together these data show that WD in huFNRG mice causes increased body weight, 197 dyslipidemia and impaired glucose metabolism compared to chow diet.

199 Western diet causes mild steatohepatitis in huFNRG mice Non-alcoholic steatohepatitis (NASH) frequently causes elevated serum transaminases ${ }^{41}$ 201 and is diagnosed histologically by the presence of hepatocyte ballooning and mononuclear 202 infiltrates in the setting of $>5 \%$ hepatocyte steatosis. ${ }^{30}$ Because the human graft rapidly developed 203 steatosis, we tested whether huFNRG mice on WD also developed features associated with 204 human NASH. 
We quantified the activities of serum transaminases ALT and AST, which were elevated in

206 huFNRG mice 4 weeks on WD compared to chow. With prolonged WD feeding, ALT activity 207 remained high while AST increased further (Fig 3a). To determine whether the ALT was produced 208 by human or mouse hepatocytes, human-specific ALT protein was quantified and this correlated 209 with serum ALT activity (Suppl Fig S3a). The human origin of transaminase activity was further 210 supported by the lack of ALT activity and only minimal increases in AST activity in muFNRG mice

211 on WD (Suppl Fig S3b). These data show that WD increased human transaminases in huFNRG 212 serum.

213 We next assessed whether human hepatocytes in huFNRG mice on WD displayed ballooning 214 degeneration, a key characteristic of $\mathrm{NASH}^{30}$ As early as 4 weeks on WD, steatotic hepatocytes 215 in huFNRG mice showed ballooning degeneration that persisted for 20 weeks (Fig 3b, Suppl Fig

216 S3c). Hepatocyte ballooning was specific to the human graft (Fig 3c) and not donor hepatocyte-

217 specific (Suppl Fig S3d). The fraction of huFNRG mice with ballooning degeneration did not 218 increase with prolonged WD feeding (Fig $\mathbf{3 d}$ ) but rather associated with the severity of steatosis 219 grade (Fig 3e). Ballooning degeneration, which remains less well-defined in mouse 220 hepatocytes, ${ }^{19}$ was not observed in either NRG or muFNRG mice on WD. Another key feature of

$221 \mathrm{NASH}$ is lobular inflammation, which is dominated by neutrophils and macrophages. ${ }^{42}$ Despite 222 their broad immunodeficient background inflammatory infiltrates were detected in huFNRG 223 mouse livers after 4 weeks on WD (Fig 3b, Suppl Fig S3c). Lobular inflammation was observed 224 in approximately one third of huFNRG mice up to 12 weeks on WD but was not observed at 20 225 weeks (Suppl Fig S3e). The livers of huFNRG mice on WD were scored according to the NAFLD 226 activity score (NAS) ${ }^{43}$ within the human graft. After 4 weeks on WD, 7 out of 8 huFNRG mice 227 developed a NAS of 3 or more. Further durations of WD did not increase the fraction of mice with 228 NAS $\geq 3$ (Fig 3f). These data show that huFNRG mice developed features of steatohepatitis 229 following WD feeding. 
Pericellular fibrosis can accompany $\mathrm{NASH}$ and is believed to result from ongoing

231 steatohepatitis. ${ }^{44}$ To test if WD feeding of huFNRG mice caused fibrosis, livers were stained for 232 collagen with Picrosirius Red (PSR). Pericellular collagen depositions were first observed in a few 233 mice after 8 weeks on WD (Fig $\mathbf{3 g}$ ), then in a larger fraction at 12 weeks and finally more prevalent 234 after 20 weeks on WD (Fig $\mathbf{3 h}$ ). Trichrome staining confirmed collagen depositions around human 235 hepatocytes, which are smaller with paler cytoplasm compared to mouse hepatocytes (Suppl Fig 236 S3f). Fibrosis was not $\mathrm{PHH} 1$ specific since mice humanized with donor $\mathrm{PHH} 2$ showed a similar 237 phenotype after 10 weeks on WD (Suppl Fig S3g). These data indicate that prolonged WD 238 feeding results in mild fibrosis around human hepatocytes.

Overall, these findings show that huFNRG mice developed features of steatohepatitis with 240 mild activity as early as 4 weeks on WD, which was accompanied by pericellular fibrosis in the 241 human graft after 8 weeks of WD feeding.

\section{WD differentially affects human and mouse liver transcriptomes over time}

In huFNRG chimeras that were created with $\mathrm{PHH}$ the non-parenchymal cells and leukocytes remained murine ( ${ }^{27}$ and unpublished observations). To determine how WD affected human and mouse gene expression we performed RNA-sequencing (RNA-seq) on livers from fasting huFNRG mice. STAR Alignment mapped $>85 \%$ of transcripts uniquely to a human reference genome (Suppl Fig S4a).

Transcripts aligned to human genome (human reads) in mice that were fed WD for 4 weeks were compared to animals on chow, which identified 662 differentially expressed transcripts (Fig 4a). Among mice on WD human transcripts changed progressively less over time, totaling 154 at 8 vs. 4 weeks, 127 at 12 vs. 8 weeks, and 95 at 20 vs. 12 weeks (Fig 4a).

254 (mouse reads). In contrast, only 2 mouse transcripts were statistically different between huFNRG 
255 mice that were fed WD or chow for 4 weeks. Transcriptional changes in the mouse reads

256 remained low for the first 12 weeks of WD feeding, but a large number of differentially expressed

257 transcripts were observed by 20 weeks (Fig 4b).

To further investigate changes in the gene expression profile we performed gene ontology

259 (GO) analysis using the GO, Kyoto Encyclopedia of Genes and Genomes (KEGG), REACTOME 260 (REAC), and Transcription Factor (TF) databases. GO (Fig 4c), KEGG and REAC (Suppl Fig

261 S4b) alignments of human reads revealed mostly downregulation of metabolic and catabolic pathways in mice 4 weeks on WD compared to chow, while transcription factors were upregulated (Suppl Fig S4b). A notable exception to many downregulated GO pathways was the Extracellular Matrix pathway, which was upregulated at 4 weeks of WD feeding. Comparing 8 vs. 4 weeks on WD identified upregulation of human GO pathways involved in cell death and NF-kB signaling. Later timepoints revealed fewer and statistically less significant pathways. Few mouse GO pathways were changed by WD feeding at early time points. At 20 weeks many pathways involved in cellular development and metabolism changed (Fig 4d, Suppl Fig S4c). Notably several metabolic pathways that were downregulated in the human transcriptome at 4 weeks were upregulated in the mouse transcriptome at 20 weeks.

Taken together, these data indicate that many metabolic pathways were transcriptionally downregulated in the human graft as early as 4 weeks on WD, while the mouse transcriptome of huFNRG mice remained largely silent until 20 weeks of WD feeding.

\section{Mice humanized with a homozygous PNPLA3-148M donor develop steatohepatitis}

Having established a new NAFLD model in chimeric mice, we set out to characterize how the PNPLA3-148M polymorphism in hepatocytes affected the phenotype. Thus far huFNRG mice were humanized with PHH donors that were homozygous for PNPLA3-148I (148I-huFNRG). We next tested how chimeric mice humanized with the PNPLA3-148M variant responded to WD. To 
280 this end Fah"- Rag2 $^{-/}$II2rg ${ }^{\text {null }}(\mathrm{FRG})$ mice $^{26}$ were humanized with a homozygous PNPLA3-148M

281 donor (148M-huFRG) and given WD for 4 weeks. As expected WD caused metabolic changes,

282 including raised fasting blood glucose, a minimal increase in fasting insulin and generally higher 283 plasma triglycerides (Suppl Fig S5a-c). Livers of 148M-huFRG mice on WD had moderate $284(>33 \%)$ and severe ( $>66 \%)$ steatosis in contrast to $<5 \%$ steatosis that was observed in chow-fed 285 mice (Fig 5a-b). Histological findings were confirmed by 5.3-fold increase in TAG concentrations 286 in the livers of 148M-huFRG mice on WD compared to chow-fed controls (Suppl Fig S5d). In 287 contrast to previous 148I-huFNRG studies, the human graft showed not only macrovesicular but 288 also microvesicular steatosis in all 148M-huFRG mice on WD (Fig 5c). In addition, 148M-huFRG 289 livers contained Mallory-Denk bodies, high grade of ballooning degeneration and lobular 290 inflammation, resulting in 4 out 5 mice with a NAS of 5 or above (Fig $5 d$, Suppl Fig S5e-f). These 291 data demonstrate that 148M-huFRG mice subjected to WD for 4 weeks showed features of 292 steatohepatitis with moderate activity.

To investigate if 148M-huFRG mice transcriptionally responded differently to WD feeding 294 than 148I-huFNRG animals, RNA-seq analyses was performed. The human graft in 148M-huFRG 295 mice responded to 4 weeks on WD with numerous transcriptomic changes compared to chow, 296 with 1458 downregulated and 1294 upregulated genes (Fig 5e). Pathway analyses revealed that 297 metabolic and catabolic pathways were mostly downregulated by WD in 148M-huFRG mice, 298 similar to previous results in 148I-huFNRG mice. Despite the similarities between both models, 299 there were pathways, including oxidative phosphorylation and mitochondrial function, which were 300 downregulated following WD only in the livers of 148M-huFRG mice but not in 148I-huFNRG. In 301 addition, some pathways were differentially upregulated by WD between these two models (Fig 302 5f). Comparing individual genes from livers of 148M-huFRG mice and 148I-huFNRG, there were 3032329 differently expressed transcripts on chow and 3268 on WD (Suppl Fig S5g). However, 
304 when comparing transcripts that changed by WD feeding over chow in each model $(\Delta$ WD), there were only 108 genes differentially regulated between these models (Fig $\mathbf{5 g}$ ).

Taken together, these findings show that 148M-huFRG mice developed microvesicular steatosis and steatohepatitis with moderate activity after 4 weeks on WD. While few pathways were transcriptionally different between 148M-huFRG and 148I-huFNRG mice on the same diet, several metabolic pathways were uniquely downregulated in 148M hepatocytes.

\section{PNPLA3-148M overexpression in hepatocytes exacerbates steatosis in mice on WD}

In addition to the PNPLA3 genotype, 148M-huFRG and 148I-huFNRG mice were distinct

313 in human hepatocyte donor background and mouse strain differences. To better characterize if

314 phenotypic differences were due to the PNPLA3 variant, we transduced mouse-passaged $\mathrm{PHH}$

315 cultures from a 148I homozygous donor (PHH1) with PNPLA3-148M and red fluorescent protein 316 (RFP), 148 I and RFP or RFP only before re-transplantation into mice ${ }^{25}$. Humanization kinetics 317 did not differ between the PNPLA3 variants or RFP controls as determined by serum hAlb values

318 (Suppl Fig S6a). Once hAlb serum levels plateaued, huFNRG mice with transduced PNPLA3148M hepatocytes (td148M), td148I or tdRFP mice were subjected to 4 weeks on WD or chow (Fig 6a). In the WD groups lentiviral PNPLA3 transduction resulted in 61-fold higher mRNA levels 321 compared to endogenous levels in tdRFP mice, while in the chow groups td148I had 2-fold higher 322 levels than td148M mice. In tdRFP mice WD caused 3.7-fold higher endogenous PNPLA3 mRNA 323 levels compared to chow (Suppl Fig S6b).

WD induced human hepatocyte steatosis in all three groups of mice, with only td148M livers containing widespread microvesicular steatosis (Fig 6b-c). Strikingly, even on chow td148M livers contained rare areas with human microvesicular steatosis, which was not observed in 327 td148I or tdRFP livers (Suppl Fig S6c). Lipid quantification in livers from mice on WD showed $32836 \%$ more triglycerides in td148M livers compared to td148I or tdRFP, with only minimal 
329 differences in mice on chow (Fig 6d). Despite these phenotypic changes, the NAS was not

330 statistically different in td148M mice from td148I or tdRFP animals (Fig 6e, Suppl Fig 6d-e).

331 These data demonstrate that PNPLA3 overexpressing huFNRG mice on WD developed 332 steatohepatitis in the human graft, with td148M livers containing widespread microvesicular 333 steatosis and increased hepatic triglycerides.

Since RNA-seq pathway analyses did not identify many differences between the $148 \mathrm{M}$ huFRG and 148I-huFNRG models (Fig 5g), we performed qRT-PCR for 80 different human 336 genes associated with fatty liver diseases (Table 1). Liver tissue from td148I and td148M mice 337 that were fed chow revealed 20 genes that were expressed differently, with lipoprotein lipase 338 showing the greatest difference (Suppl Fig S6f). Comparing both groups following WD we 339 identified only 8 genes that were affected by 2-fold or less, of which CYP2E1 was most 340 transcriptionally upregulated in td148M livers (Fig 6f). These results illustrate the modest 341 expression differences between mice overexpressing the two PNPLA3 variants on WD.

342 Combined, these data show that PNPLA3-148M overexpression in hepatocytes on a homozygous 343148 l background caused widespread microvesicular steatosis and increased liver triglycerides 344 after 4 weeks of WD feeding.

353 in widespread microvesicular steatosis and Mallory Denk bodies (Fig 7c), which were not 
354 observed in either td148I or tdRFP mice. Ballooning degeneration in mice fed a HFD trended 355 toward being more severe in the td148M group than in tdRFP and td148I $(p=0.063$, Suppl Fig 356 S7b) while lobular inflammation was not affected by PNPLA3 overexpression (Suppl Fig S7c). 357 Driven by steatosis and ballooning degeneration grades, the NAS was higher in td148M than in 358 td148I or tdRFP mice (Fig 7d). These data show that HFD caused steatohepatitis in PNPLA3 359 transduced human grafts, with $148 \mathrm{M}$ transduction resulting in microvesicular steatosis, Mallory 360 Denk bodies and higher activity compared to 1481.

361 PNPLA3 mRNA levels in liver pieces of td148I and td148M mice on HFD were similar (Suppl Fig 362 S7d). Since lentiviral PHH transduction results in heterogeneous expression ${ }^{25}$ we next analyzed 363 whether steatosis correlated to the transgene expression, using the RFP reporter as a surrogate 364 marker. ${ }^{45}$ Low magnification fluorescence imaging of liver cross-sections stained for neutral lipids 365 suggested that td148M livers on HFD contained more lipids than td148I mice (Fig 7e). At higher

375 Combined, these findings show that PNPLA3-148M overexpression in hepatocytes from a 376 homozygous 148 I donor increased steatosis and steatohepatitis activity in chimeric mice on HFD. 


\section{Discussion}

In this study, we have established systems to model NAFLD genetics in human hepatocytes. The most striking phenotype in the 'standard' 148I-huFNRG model was the rapid onset of human steatosis on WD. This contrasted to the minimal steatosis in the residual mouse hepatocytes of huFNRG mice or in muFNRG controls. Both resembled non-humanized mouse models that require prolonged WD feeding to develop moderate steatosis. ${ }^{46}$ Impaired cross-talk between human hepatocytes and murine cells likely contributed, as we previously showed for FGF15 and FGF19 signaling, ${ }^{47}$ yet mouse and human hepatocytes may also inherently respond differently to overnutrition. This is supported by the clinical observations of how rapidly hepatic fat content changes depending on the type and amount of food intake. For example, a three-week overfeeding study increased intrahepatic triglycerides up to $55 \%{ }^{48}$ and a carbohydrate-restricted diet reduced hepatic fat by $44 \%$ in two weeks. ${ }^{49}$ These clinical data illustrate that hepatic steatosis dynamically responds to nutritional variations. huFNRG mice can provide a platform to explore how hepatocytes rapidly respond to hypercaloric diets.

Hepatocytes are central to systemic lipid and glucose regulation. Similar to previous reports $38-40$ huFNRG on chow had starkly different lipid profiles than muFNRG, in part due to mice lacking cholesterol ester transfer protein. ${ }^{40}$ In addition, we show that fasting glucose and possibly fasting insulin levels were higher in huFNRG mice than muFNRG mice on chow. Species incompatibilities may contribute to the elevated fasting glucose of huFNRG mice on chow, as was illustrated by studies in the UPA liver chimeric model..$^{50}$ While some human donors develop spontaneous steatosis in these chimeras, similar to what we observed with $\mathrm{PHH}$, administration of human growth hormone reversed this phenotype, possibly through IGF-1 signaling in the liver. ${ }^{50,51}$ We speculate that hepatocytes in huFNRG are likewise predisposed to steatosis, and therefore may only require a short duration of hypercaloric feeding to develop moderate steatosis. Interestingly, muFNRG controls without hepatic steatosis developed glucose intolerance on WD 
402 that was proportionally similar to huFNRG mice with moderate steatosis. Uncoupling hepatocyte

403 steatosis from glucose homeostasis in these models may advance insights into clinically distinct

404 forms of NAFLD with and without insulin resistance. ${ }^{52}$

Despite their broad immunodeficiency huFNRG mice displayed the features that define human $\mathrm{NASH}$, including ballooning degeneration and lobular infiltrates. This illustrates that steatohepatitis and pericellular fibrosis can be initiated without lymphocytes. Previous NAFLD studies in T cell deficient mouse strains showed these to be resistant to steatosis development, ${ }^{32}$, ${ }^{33}$ which are in line with our muFNRG negative controls and further highlight species differences between human and mouse hepatocytes. The cellular immune contributions to human NASH

412 and T cells whereas lobular infiltrates are dominated by macrophages and neutrophils. ${ }^{42}$ How

413 these cell types and their subsets are involved in the immunopathogenesis of human NASH 414 remains under investigation. Proposed mechanisms are largely based on animal models with 415 unclear translational value..$^{53-55}$ Some compounds that effectively interfered with the development 416 of murine steatohepatitis failed in NASH patients, ${ }^{56}$ and no immunological interventions have yet 417 shown clear benefits in later phase clinical trials. Furthermore, species differences preclude 418 investigations into non-conserved pathways, e.g. IL-32 that lacks a mouse orthologue. This 419 cytokine was recently associated with advanced NAFLD ${ }^{57}$ and IL32 was transcriptionally 420 upregulated in 148I-huFNRG livers after 12 weeks and 148M-huFRG livers after 4 weeks of WD 421 feeding. Going forward, studies dissecting the roles of T cells and other lymphocytes will require 422 immune competent mouse models while human immune system mice can be combined with liver 423 chimeras ${ }^{27,58}$ for investigations into pathways that are not conserved between mice and humans.

Transcriptomic characterization of huFNRG livers showed a large number of human metabolic pathways that were transcriptionally downregulated after 4 weeks of WD feeding and only a few pathways that were upregulated. Eight weeks of WD feeding caused upregulation of a 
427 small number of cellular stress and cell death pathways, and remarkably few human pathways 428 transcriptionally changed after that. Equally surprising were the small number of mouse 429 transcripts that changed during the first 12 weeks on WD. This may be partly explained by under430 sampling of mouse hepatocytes at earlier timepoints, as illustrated by changes in mouse 431 metabolic pathways after 20 weeks WD when humanization histologically appeared to decline. 432 The murine transcriptome, however, was also largely unperturbed by WD feeding. Whether 433 changes from WD were masked by cyclical nitisinone withdrawal or if non-parenchymal cells did 434 not transcriptionally respond to signals from the human graft will require further studies, including 435 in other chimera models without cyclical liver injury.

These systems will now allow for mechanistic studies into genetic variants that drive NAFLD. Many polymorphisms associated with elevated transaminases ${ }^{59}$ and most variants implicated in advanced NAFLD ${ }^{60}$ are in genes expressed in hepatocytes. As a proof-of-principle, we modeled the PNPLA3-148M polymorphism. ${ }^{6}$ Its prevalent allele frequency allowed for the 440 identification of a high-quality homozygous $148 \mathrm{M}$ donor to create human chimeras. PNPLA3 441 overexpression in homozygous 148 I hepatocytes then controlled for genetic differences between 442 these two PHH donors. Only PNPLA3-148M overexpression resulted in microvesicular steatosis, 443 suggesting that this phenotype in 148M-huFRG mice is associated with its PNPLA3 genotype. 444 Differences between $148 \mathrm{I}$ and $148 \mathrm{M}$ had previously been identified in mouse hepatocytes and 445 human cell lines, ${ }^{10,11,13,15,16,61}$ none of which displayed microvesicular steatosis. By contrast, 446 hepatocytes in arguably the most physiological mouse model, namely in PNPLA3 148M knockin 447 mice, contained increased lipid droplet sizes on hypercaloric diet. ${ }^{11}$ This suggests that the 448 microvesicular phenotype, which clinically correlates with advanced $\mathrm{NASH},{ }^{62}$ may be a humanspecific 148M hepatocyte response to hypernutrition.

Taken together these results show the utility of liver chimeras for studies of pathways that 451 are genetically implicated in human fatty liver diseases. Notwithstanding their broad implications 
452 to advance human hepatocyte research, several technical challenges remain. Identifying high-

453 quality PHH donors with rare allele variants, e.g. in $T M 6 S F 2$ or $M B O A T 7,63,64$ will remain difficult. 454 And creating isogenic controls for more frequent variants, e.g. in PNPLA3 and HSD17B13, ${ }^{65}$ will 455 require improvements in gene editing efficiency of primary human materials, either to disrupt 456 genes or prime edit polymorphisms. ${ }^{66}$ An alternative strategy is to prime edit renewable cells such 457 as pluripotent stem cells. However, hepatocyte-like cells derived from these sources have thus 458 far failed to reliably and reproducibly humanize mouse chimera models. ${ }^{67,68}$ We here used an 459 overexpression system to control for human hepatocyte donor differences. The lentiviral LTR 460 promotor $^{69}$ in our models failed to recapitulate the complexities of dietary regulation of PNPLA3 461 transcription and translation. ${ }^{20}$ This likely resulted in supraphysiological protein amounts 462 particularly of the $148 \mathrm{M}$ variant with its known resistance to ubiquitylation, ${ }^{11,12,15}$ and may explain 463 the steatotic areas observed in td148M mice on chow. Human and mouse PNPLA3 expression 464 are regulated by sterol regulatory binding protein 1c (SREBP1c), ${ }^{20,} 22,23$ while mouse Pnpla3 465 transcription may additionally be under control of carbohydrate response element binding protein 466 (ChREBP). ${ }^{22}$ Creating human liver chimeric mice with PNPLA3 expression under SREBP1c 467 regulation would become a more physiological system to study PNPLA3-148M in hepatocytes 468 with appropriate controls. Going forward, overcoming technical challenges with primary 469 hepatocyte gene editing and hepatocyte-like cell engraftment would further advance the models 470 presented here and generate ever more physiologically relevant systems to dissect the 471 pathophysiology of human fatty liver diseases. 


\section{REFERENCES}

474 1. Younossi, Z.M. et al. Global epidemiology of nonalcoholic fatty liver disease-Meta-analytic

2. Yki-Järvinen, H. Non-alcoholic fatty liver disease as a cause and a consequence of metabolic syndrome. Lancet Diabetes Endocrinol 2, 901-910 (2014).

3. Diehl, A.M. \& Day, C. Cause, Pathogenesis, and Treatment of Nonalcoholic Steatohepatitis. N Engl J Med 377, 2063-2072 (2017).

4. Buzzetti, E., Pinzani, M. \& Tsochatzis, E.A. The multiple-hit pathogenesis of non-alcoholic fatty liver disease (NAFLD). Metabolism 65, 1038-1048 (2016).

5. Romeo, S., Sanyal, A. \& Valenti, L. Leveraging Human Genetics to Identify Potential New Treatments for Fatty Liver Disease. Cell Metab 31, 35-45 (2020).

6. Romeo, S. et al. Genetic variation in PNPLA3 confers susceptibility to nonalcoholic fatty liver disease. Nat Genet 40, 1461-1465 (2008).

7. Trépo, E., Romeo, S., Zucman-Rossi, J. \& Nahon, P. PNPLA3 gene in liver diseases. J Hepatol 65, 399-412 (2016).

8. Pingitore, P. \& Romeo, S. The role of PNPLA3 in health and disease. Biochim Biophys Acta Mol Cell Biol Lipids 1864, 900-906 (2019).

9. Tardelli, M., Bruschi, F.V. \& Trauner, M. The role of metabolic lipases in the pathogenesis and management of liver disease. Hepatology (2020).

10. Li, J.Z. et al. Chronic overexpression of PNPLA3I148M in mouse liver causes hepatic steatosis. J Clin Invest 122, 4130-4144 (2012).

11. Smagris, E. et al. Pnpla3/148M knockin mice accumulate PNPLA3 on lipid droplets and develop hepatic steatosis. Hepatology 61, 108-118 (2015).

12. Lindén, D. et al. Pnpla3 silencing with antisense oligonucleotides ameliorates nonalcoholic steatohepatitis and fibrosis in Pnpla3 I148M knock-in mice. Mol Metab 22, 49-61 (2019). 
498 13. Huang, Y., Cohen, J.C. \& Hobbs, H.H. Expression and characterization of a PNPLA3 protein isoform (I148M) associated with nonalcoholic fatty liver disease. J Biol Chem 286, 37085-37093 (2011).

14. Kumari, M. et al. Adiponutrin functions as a nutritionally regulated lysophosphatidic acid acyltransferase. Cell Metab 15, 691-702 (2012).

15. BasuRay, S., Smagris, E., Cohen, J.C. \& Hobbs, H.H. The PNPLA3 variant associated with fatty liver disease (I148M) accumulates on lipid droplets by evading ubiquitylation. Hepatology 66, 1111-1124 (2017).

16. Wang, Y., Kory, N., BasuRay, S., Cohen, J.C. \& Hobbs, H.H. PNPLA3, CGI-58, and Inhibition of Hepatic Triglyceride Hydrolysis in Mice. Hepatology 69, 2427-2441 (2019).

17. Yang, A., Mottillo, E.P., Mladenovic-Lucas, L., Zhou, L. \& Granneman, J.G. Dynamic interactions of ABHD5 with PNPLA3 regulate triacylglycerol metabolism in brown adipocytes. Nat Metab 1, 560-569 (2019).

18. Wattacheril, J., Issa, D. \& Sanyal, A. Nonalcoholic Steatohepatitis (NASH) and Hepatic Fibrosis: Emerging Therapies. Annu Rev Pharmacol Toxicol 58, 649-662 (2018).

513 19. Farrell, G. et al. Mouse Models of Nonalcoholic Steatohepatitis: Toward Optimization of

Their Relevance to Human Nonalcoholic Steatohepatitis. Hepatology 69, 2241-2257 (2019).

20. Huang, Y. et al. A feed-forward loop amplifies nutritional regulation of PNPLA3. Proc Natl Acad Sci U S A 107, 7892-7897 (2010).

21. Lake, A.C. et al. Expression, regulation, and triglyceride hydrolase activity of Adiponutrin family members. J Lipid Res 46, 2477-2487 (2005).

22. Dubuquoy, C. et al. Distinct regulation of adiponutrin/PNPLA3 gene expression by the transcription factors ChREBP and SREBP1c in mouse and human hepatocytes. $J$ Hepatol 55, 145-153 (2011). 
523 23. Perttilä, J. et al. PNPLA3 is regulated by glucose in human hepatocytes, and its I148M mutant slows down triglyceride hydrolysis. Am J Physiol Endocrinol Metab 302, E10631069 (2012).

24. de Jong, Y.P. et al. Broadly neutralizing antibodies abrogate established hepatitis $C$ virus infection. Sci Trans/ Med 6, 254ra129 (2014).

25. Michailidis, E. et al. Expansion, in vivo-ex vivo cycling, and genetic manipulation of primary human hepatocytes. Proc Natl Acad Sci U S A 117, 1678-1688 (2020).

26. Azuma, H. et al. Robust expansion of human hepatocytes in Fah-//Rag2-///l2rg-/- mice. Nat Biotechnol 25, 903-910 (2007).

27. Billerbeck, E. et al. Humanized mice efficiently engrafted with fetal hepatoblasts and syngeneic immune cells develop human monocytes and NK cells. J Hepatol 65, 334-343 (2016).

28. Kawahara, T. et al. Factors affecting hepatocyte isolation, engraftment, and replication in an in vivo model. Liver Transp/ 16, 974-982 (2010).

29. Vanwolleghem, T. et al. Factors determining successful engraftment of hepatocytes and susceptibility to hepatitis B and C virus infection in UPA-SCID mice. J Hepatol 53, 468-476 (2010).

30. Yeh, M.M. \& Brunt, E.M. Pathological features of fatty liver disease. Gastroenterology 147, 754-764 (2014).

31. Grompe, M. et al. Pharmacological correction of neonatal lethal hepatic dysfunction in a murine model of hereditary tyrosinaemia type I. Nat Genet 10, 453-460 (1995).

32. Bhattacharjee, J. et al. Role of immunodeficient animal models in the development of fructose induced NAFLD. J Nutr Biochem 25, 219-226 (2014).

33. Sun, G. et al. OX40 Regulates Both Innate and Adaptive Immunity and Promotes Nonalcoholic Steatohepatitis. Cell Rep 25, 3786-3799.e3784 (2018). 
548 34. Liu, X. et al. Lack of mature lymphocytes results in obese but metabolically healthy mice when fed a high-fat diet. Int J Obes (Lond) 39, 1548-1557 (2015).

35. Hasegawa, M. et al. The reconstituted 'humanized liver' in TK-NOG mice is mature and functional. Biochem Biophys Res Commun 405, 405-410 (2011).

45. Kane, M. et al. Identification of Interferon-Stimulated Genes with Antiretroviral Activity. Cell Host Microbe 20, 392-405 (2016). 
572 46. Ito, M. et al. Longitudinal analysis of murine steatohepatitis model induced by chronic exposure to high-fat diet. Hepatol Res 37, 50-57 (2007).

47. Naugler, W.E. et al. Fibroblast Growth Factor Signaling Controls Liver Size in Mice With Humanized Livers. Gastroenterology 149, 728-740.e715 (2015).

48. Luukkonen, P.K. et al. Saturated Fat Is More Metabolically Harmful for the Human Liver Than Unsaturated Fat or Simple Sugars. Diabetes Care 41, 1732-1739 (2018).

49. Mardinoglu, A. et al. An Integrated Understanding of the Rapid Metabolic Benefits of a Carbohydrate-Restricted Diet on Hepatic Steatosis in Humans. Cell Metab 27, 559$571 . e 555$ (2018).

50. Tateno, C. et al. Growth hormone-dependent pathogenesis of human hepatic steatosis in a novel mouse model bearing a human hepatocyte-repopulated liver. Endocrinology 152, 1479-1491 (2011).

51. Yakar, S. et al. Inhibition of growth hormone action improves insulin sensitivity in liver IGF1-deficient mice. J Clin Invest 113, 96-105 (2004).

52. Luukkonen, P.K. et al. Hepatic ceramides dissociate steatosis and insulin resistance in patients with non-alcoholic fatty liver disease. J Hepatol 64, 1167-1175 (2016).

53. Cai, J., Zhang, X.J. \& Li, H. The Role of Innate Immune Cells in Nonalcoholic Steatohepatitis. Hepatology 70, 1026-1037 (2019).

54. Kazankov, K. et al. The role of macrophages in nonalcoholic fatty liver disease and nonalcoholic steatohepatitis. Nat Rev Gastroenterol Hepatol 16, 145-159 (2019).

592 55. Sutti, S. \& Albano, E. Adaptive immunity: an emerging player in the progression of NAFLD. Nat Rev Gastroenterol Hepatol 17, 81-92 (2020).

594 56. Ratziu, V. et al. Cenicriviroc Treatment for Adults With Nonalcoholic Steatohepatitis and 595 Fibrosis: Final Analysis of the Phase 2b CENTAUR Study. Hepatology (2020). 
596 57. Baselli, G.A. et al. Liver transcriptomics highlights interleukin-32 as novel NAFLD-related cytokine and candidate biomarker. Gut 69, 1855-1866 (2020).

598 58. Wilson, E.M. et al. Extensive double humanization of both liver and hematopoiesis in

59. Chambers, J.C. et al. Genome-wide association study identifies loci influencing concentrations of liver enzymes in plasma. Nat Genet 43, 1131-1138 (2011).

60. Eslam, M., Valenti, L. \& Romeo, S. Genetics and epigenetics of NAFLD and NASH: Clinical impact. J Hepatol 68, 268-279 (2018).

61. Luukkonen, P.K. et al. Human PNPLA3-I148M variant increases hepatic retention of polyunsaturated fatty acids. JCI Insight 4 (2019).

62. Tandra, S. et al. Presence and significance of microvesicular steatosis in nonalcoholic fatty liver disease. J Hepatol 55, 654-659 (2011).

63. Kozlitina, J. et al. Exome-wide association study identifies a TM6SF2 variant that confers susceptibility to nonalcoholic fatty liver disease. Nat Genet 46, 352-356 (2014).

64. Mancina, R.M. et al. The MBOAT7-TMC4 Variant rs641738 Increases Risk of Nonalcoholic Fatty Liver Disease in Individuals of European Descent. Gastroenterology 150, 12191230.e1216 (2016).

65. Abul-Husn, N.S. et al. A Protein-Truncating HSD17B13 Variant and Protection from Chronic Liver Disease. N Engl J Med 378, 1096-1106 (2018).

66. Anzalone, A.V. et al. Search-and-replace genome editing without double-strand breaks or donor DNA. Nature 576, 149-157 (2019).

67. Chen, C., Soto-Gutierrez, A., Baptista, P.M. \& Spee, B. Biotechnology Challenges to In Vitro Maturation of Hepatic Stem Cells. Gastroenterology 154, 1258-1272 (2018). 
619 68. Luce, E., Messina, A., Duclos-Vallée, J.C. \& Dubart-Kupperschmitt, A. Advanced techniques and awaited clinical applications for human pluripotent stem cell differentiation into hepatocytes. Hepatology (2021).

69. Schoggins, J.W. et al. A diverse range of gene products are effectors of the type I interferon antiviral response. Nature 472, 481-485 (2011).

70. Santoro, N. et al. A common variant in the patatin-like phospholipase 3 gene (PNPLA3) is associated with fatty liver disease in obese children and adolescents. Hepatology $\mathbf{5 2}$, $1281-1290$ (2010).

71. Donati, B. et al. The rs2294918 E434K variant modulates patatin-like phospholipase domain-containing 3 expression and liver damage. Hepatology 63, 787-798 (2016).

72. Steensels, S. et al. Acot9 traffics mitochondrial short-chain fatty acids towards de novo lipogenesis and glucose production in the liver. Hepatology (2020).

74. Alves-Bezerra, M. et al. Thioesterase Superfamily Member 2 Promotes Hepatic VLDL Secretion by Channeling Fatty Acids Into Triglyceride Biosynthesis. Hepatology 70, 496510 (2019).

73. Hyogo, H., Roy, S., Paigen, B. \& Cohen, D.E. Leptin promotes biliary cholesterol elimination during weight loss in ob/ob mice by regulating the enterohepatic circulation of bile salts. J Biol Chem 277, 34117-34124 (2002).

75. Guilbert, M.C., Therrien, A., Soucy, G., Trudel, D. \& Nguyen, B.N. Nodular Regenerative Hyperplasia: Expression Pattern of Glutamine Synthetase and a Potential Role for Hepatic Progenitor Cells. Appl Immunohistochem Mol Morphol 28, 243-248 (2020).

76. Schindelin, J. et al. Fiji: an open-source platform for biological-image analysis. Nat Methods 9, 676-682 (2012).

77. Deroulers, C. et al. Analyzing huge pathology images with open source software. Diagn Pathol 8, 92 (2013). 
644 78. Ritchie, M.E. et al. limma powers differential expression analyses for RNA-sequencing and microarray studies. Nucleic Acids Res 43, e47 (2015).

646 79. Raudvere, U. et al. g:Profiler: a web server for functional enrichment analysis and conversions of gene lists (2019 update). Nucleic Acids Res 47, W191-W198 (2019).

648 80. Dobin, A. et al. STAR: ultrafast universal RNA-seq aligner. Bioinformatics 29, 15-21 (2013).

649 81. Anders, S., Pyl, P.T. \& Huber, W. HTSeq--a Python framework to work with highthroughput sequencing data. Bioinformatics 31, 166-169 (2015).

651 82. Liberzon, A. et al. The Molecular Signatures Database (MSigDB) hallmark gene set collection. Cell Syst 1, 417-425 (2015). 


\section{FigURE LEGENDS}

\section{Figure 1: Human hepatocytes in chimeric mice on WD rapidly develop steatosis}

663 a) Experimental timeline of FNRG mice that were transplanted with primary human hepatocytes $664(\mathrm{PHH})$, cycled off nitisinone to create huFNRG mice, and then subjected to chow or WD for up to 66512 weeks.

666 b) H\&E staining on livers from huFNRG mice on chow and after 4 or 12 weeks on WD.

667 c) Staining against human nuclear mitotic apparatus- 1 in livers of huFNRG mice on chow and 4 668 or 12 weeks on WD. Areas with predominantly mouse hepatocytes are outlined.

669 d) Staining against human glutamine synthetase on livers from huFNRG mice on chow and 4 670 weeks on WD. Central vein (CV), portal tract (PT).

671 e) Liver steatosis score of the human graft in huFNRG mice on chow and after 4 to 20 weeks on

672 WD. Symbols individual mice, bars are median, unpaired $t$-test with ${ }^{* *} \mathrm{p}<0.005,{ }^{* *} \mathrm{p}<0.0005$ and $673^{* * * *} p<0.0001$

674 f) Hepatic triglyceride levels in livers of huFNRG mice on chow and after 4 to 12 weeks on WD. 675 Symbols individual mice, bars are median, unpaired $t$-test with ${ }^{* * *} \mathrm{p}<0.0005$ and ${ }^{* * *} \mathrm{p}<0.0001$. 676 g) H\&E staining on livers from muFNRG mice on chow and after 4 or 12 weeks on WD.

a) Serum human albumin (hAlb) levels in huFNRG mice during the course of chow and WD 680 feeding.

681 b) Oil Red O staining on livers from huFNRG mice on chow and after 4 weeks on WD.

682 c) Hepatic cholesterol quantification from huFNRG mice on chow and after 4 to 12 weeks on WD. 683 Symbols individual mice, bars are median, unpaired $t$-test with ${ }^{*} \mathrm{p}<0.05,{ }^{* *} \mathrm{p}<0.005$. 
d) Hepatic triglyceride levels versus clinical steatosis scoring of the human graft in huFNRG mice

685 on chow and after 4 to 12 weeks on WD. Symbols individual mice, line is simple linear regression, $686 r^{2}$ Pearson correlation coefficient.

687 e) H\&E staining on livers from NRG mice on chow and after 12 weeks on WD.

688 f) Hepatic triglyceride quantification from muFNRG mice on chow, and after 4 and 12 weeks on 689 WD. Symbols individual mice, bars are median.

690 g) Liver sections from PHH2 huFNRG mice on chow and after 10 weeks on WD stained for human 691 nuclear mitotic apparatus-1 (left). Liver sections from PHH2 huTK-NOG mice on chow and after 69210 weeks on WD stained for H\&E (right).

693 h) H\&E staining on livers from PHH3 huFNRG and PHH3 hu-uPA/SCID mice livers. High 694 magnification images of a huFNRG mouse liver on chow (upper left); after 4 weeks on WD (lower 695 left); low and high magnification images of a hu-uPA/SCID mouse liver on chow (right).

697 Figure 2: Systemic metabolic effects of WD in chimeric FNRG mice

698 a) Body weight in huFNRG mice during time course of chow or WD feeding. Symbols individual 699 mice, line is mean, two-way ANOVA Sidak pairwise comparison ${ }^{*} p<0.05,{ }^{* *} p<0.005,{ }^{* * *} p<0.0005$.

700 b) Gonadal fat fraction of total body weight in huFNRG mice on chow and after 4 to 20 weeks on 701 WD. Symbols individual mice, bars are median, unpaired $t$-test with ${ }^{*} p<0.05,{ }^{* *} p<0.005$, $702^{* * *} p<0.0005$

703 c) Cholesterol in plasma lipoprotein fractions from huFNRG and muFNRG mice on chow and 704 after 4 weeks on WD. Pooled plasma (3 mice/group), symbols are technical means.

705 d) Triglycerides in plasma lipoprotein fractions from huFNRG and muFNRG mice on chow and 706 after 4 weeks on WD. Pooled plasma (3 mice/group), symbols are technical means.

707 e) Fasting blood glucose from huFNRG and muFNRG mice on chow and after 4 weeks on WD. 708 Symbols individual mice, lines are median, unpaired $t$-test ${ }^{* * *} \mathrm{p}<0.0001$. 
709 f) Fasting serum mouse insulin from huFNRG and muFNRG mice on chow and after 4 weeks on

710 WD. Symbols individual mice, lines are median, unpaired $t$-test with ${ }^{*} \mathrm{p}<0.05,{ }^{* \star} \mathrm{p}<0.005$.

711 g) Intraperitoneal glucose, insulin and pyruvate tolerance testing in huFNRG and muFNRG mice 712 on chow and after 4 weeks on WD. Blood glucose values measured at baseline (0) and at 713 indicated timepoints after i.p. injections with each agent. Symbols are mean \pm SEM of 5-7 mice 714 per group, two-way ANOVA Sidak pairwise comparison of huFNRG on chow and WD ( $p$-values 715 on top) or muFNRG on chow and WD ( $p$-values in bottom), ${ }^{*} p<0.05,{ }^{* *} p<0.005,{ }^{* *} p<0.0005$ and $716^{* * * *} \mathrm{p}<0.0001$

\section{Suppl Figure S2: Systemic metabolic effects of WD feeding}

719 a) Cholesterol quantification in plasma lipoprotein fractions from huFNRG and muFNRG mice on 720 chow and after 4 weeks on WD. Pooled plasma (3 mice/group), column bars are technical means $721 \pm$ SEM

b) Triglyceride quantification in plasma lipoprotein fractions from huFNRG and muFNRG mice on

723 chow and after 4 weeks on WD. Pooled plasma (3 mice/group), column bars are technical means $724 \pm$ SEM.

725 c) Intraperitoneal glucose tolerance testing in huFNRG on chow and after 8 weeks on WD. 726 Symbols are mean \pm SEM of 5-8 mice per group, two-way ANOVA Sidak pairwise comparison 727 with ${ }^{*} p<0.05,{ }^{* *} p<0.005,{ }^{* *} p<0.0005$ and ${ }^{* * *} p<0.0001$

728 d) Mouse insulin secretion during glucose tolerance testing in huFNRG mice on chow and after 7298 weeks on WD. Symbols are mean \pm SEM of 5-8 mice per group.

731 Figure 3: huFNRG mice on WD develop mild steatohepatitis

732 a) ALT and AST activity in serum from huFNRG mice on chow and after 4 to 12 weeks on WD. 733 Symbols individual mice, bars are median, unpaired $t$-test ${ }^{* *} \mathrm{p}<0.005,{ }^{* * *} \mathrm{p}<0.0001$. 
734 b) H\&E staining from a huFNRG mouse liver after 8 weeks on WD. Black arrow indicates hepatocyte ballooning degeneration and white arrow indicates lobular inflammation.

c) Staining against human nuclear mitotic apparatus- 1 in the liver of a huFNRG mouse after 4 weeks on WD. Black arrow indicates hepatocyte ballooning.

d) Fractions of huFNRG mice with human hepatocyte ballooning degeneration on chow and WD over time. Number of mice with ballooning per group at bottom of bars.

e) Fractions of huFNRG mice with human hepatocyte ballooning degeneration by grade of steatosis. Number of mice with ballooning per group at bottom of bars.

f) NAFLD activity score (NAS) in the human graft of huFNRG mice on chow and after 4 to 20 weeks on WD. Symbols individual mice, bars are median, unpaired $t$-test ${ }^{* *} p<0.005$, ${ }^{* * *} \mathrm{p}<0.0001$.

g) Picosirius Red staining for collagen in livers from huFNRG mice on chow and after 4 to 20 weeks on WD.

h) Fibrosis stages in the human graft of huFNRG liver on chow and after 4 to 20 weeks on WD. Mouse numbers at bottom of bars.

\section{Suppl Figure S3: Mild steatohepatitis in huFNRG mice on WD}

a) Human ALT protein versus ALT activity in serum of huFNRG mice on chow and WD. Symbols individual mice, line is simple linear regression, $r^{2}$ Pearson correlation coefficient.

b) ALT and AST activity in serum of muFNRG mice on chow and after 4 to 12 weeks on WD. Symbols individual mice, bars are median, unpaired $t$-test with ${ }^{* *} \mathrm{p}<0.005,{ }^{* * *} \mathrm{p}<0.0001$.

c) H\&E staining on livers from huFNRG mice after 4, 12 and 20 weeks on WD. Black arrow indicates hepatocyte ballooning degeneration and white arrow indicated lobular inflammation.

d) H\&E staining on liver from a huFNRG mouse engrafted with PHH3 after 4 weeks on WD. Black arrow indicates hepatocyte ballooning degeneration. 
759 e) Fractions of huFNRG mice with lobular inflammation on chow and after 4 to 20 weeks on WD.

760 Number of mice with lobular inflammation per group at bottom of bars.

761 f) Masson trichrome staining for collagen on livers from huFNRG mice on chow and after 12 and 76220 weeks on WD.

763 g) Picosirius Red staining for collagen on a liver of huFNRG mouse engrafted with $\mathrm{PHH} 2$ after 10 764 weeks on WD.

Figure 4: Transcriptional changes in huFNRG mice on WD

a) Volcano plots of liver transcripts mapped to human genome (human reads) comparing 768 huFNRG mice 4 weeks on WD to chow, and longer WD durations to the previous WD timepoint. 769 RNA-sequencing of 3-4 mice per group, red symbols FDR $<0.05$, grey denotes not significant.

b) Volcano plots of liver transcripts mapped to mouse genome (mouse reads) comparing 771 huFNRG mice 4 weeks on WD to chow, and longer WD durations to the previous WD timepoint.

772 RNA-sequencing of 3-4 mice per group, red symbols FDR $<0.05$, grey denotes not significant.

773 c) Gene-ontology (GO) pathway analysis of human reads from livers of huFNRG mice 4 weeks 774 on WD to chow, and longer WD durations to the previous WD timepoint. Blue symbols are down775 and red symbols are upregulated pathways, grey denotes no significant changes, symbol size 776 indicates statistical significance.

777 d) Gene-ontology (GO) pathway analysis of mouse reads from livers of huFNRG mice 4 weeks 778 on WD to chow, and longer WD durations to the previous WD timepoint. Blue symbols are down779 and red symbols are upregulated pathways, grey denotes no significant changes, symbol size 780 indicates statistical significance. 
783 a) Spliced Transcripts Alignment to a Reference (STAR) Scores of transcripts from fasting 784 huFNRG mice livers on chow and after 4 to 20 weeks on WD aligned to human reference genome. 785 Numbers on left indicate individual mouse tags.

786 b) Manhattan plots comparing human pathways in livers from huFNRG mice 4 weeks on WD to 787 chow, and longer WD durations to the previous WD timepoint. Gene-ontology (GO) with GO-MF 788 (Molecular Function), GO-CC (Cellular Component), GO-BP (Biological Process), Kyoto 789 Encyclopedia of Genes and Genomes (KEGG), REACTOME (REAC) and Transcription Factor 790 (TF). Symbol size indicates percentage of statistically significant differently expressed transcripts 791 aligned to a certain pathway, only pathways statistically significant enriched for a group of mice 792 are displayed.

793 c) Manhattan plots comparing mouse pathways in livers from huFNRG mice 4 weeks on WD to 794 chow, and longer WD durations to the previous WD timepoint. Gene-ontology (GO) with GO-MF 795 (Molecular Function), GO-CC (Cellular Component), GO-BP (Biological Process), Kyoto 796 Encyclopedia of Genes and Genomes (KEGG), REACTOME (REAC) and Transcription Factor 797 (TF). Symbol size indicates percentage of statistically significant differently expressed transcripts 798 aligned to a certain pathway, only pathways statistically significant enriched for a group of mice 799 are displayed.

801 Figure 5) PNPLA3-148M huFRG mice on WD develop steatohepatitis

802 a) H\&E staining on livers from 148M-huFRG mice on chow and after 4 weeks on WD.

803 b) Clinical steatosis score of the human graft on livers from 148M-huFNRG mice on chow and 804 after 4 weeks WD. Symbols are individual mice, bars median, unpaired $t$-test ${ }^{* *} p<0.0005$.

805 c) H\&E staining on livers from 148M-huFRG mice 4 weeks on WD. Grey arrow indicates Mallory 806 Denk body, black arrow ballooning degeneration and white arrow lobular inflammation. 
807 d) NAFLD Activity Score (NAS) in the human graft of 148M-huFRG mice on chow and after 4 808 weeks WD. Symbols individual mice, bars are median, unpaired $t$-test ${ }^{* * *} \mathrm{p}<0.0005$.

809 e) Volcano plots of liver transcripts mapped to human genome (human reads) comparing 148M810 huFRG mice after 4 weeks WD vs. chow. RNA-sequencing of 3-4 mice per group, red symbols 811 FDR $<0.05$, grey denotes not significant.

812 f) Gene-ontology (GO) pathway analysis of human reads comparing 148I-huFNRG and 148M813 huFRG livers after 4 weeks WD vs. chow. Blue symbols are down- and red symbols are 814 upregulated pathways, grey denotes no significant changes, symbol size indicates statistical 815 significance.

816 g) Volcano plots of human reads differentially expressed after 4 weeks WD compared to chow $(\Delta$ 817 WD) in 148M-huFRG livers versus 148I-huFNRG livers. Red symbols FDR <0.05, grey denotes 818 not significant.

820 Suppl Figure S5: 148M-huFRG mice develop steatohepatitis

821 a) Fasting blood glucose from 148M-huFRG mice on chow and after 4 weeks on WD. Symbols 822 individual mice, bars are median, unpaired t-test, ${ }^{* *} \mathrm{p}<0.005$.

b) Fasting serum mouse insulin in 148M-huFRG mice on chow and after 4 weeks on WD.

824 Symbols individual mice, bars are median.

825 c) Fasting plasma triglycerides in $148 \mathrm{M}$-huFRG mice on chow and after 4 weeks on WD.

826 Symbols individual mice, bars are median.

827 d) Hepatic triglyceride quantification in 148M-huFRG mice on chow and after 4 weeks on WD.

e) Left: Fractions of huFRG mice with human hepatocyte ballooning degeneration on chow and 8304 weeks on WD. Number of mice with ballooning per group at bottom of bars. Right: Ballooning 
831 score in the human graft of 148M-huFRG mice on chow and after 4 weeks of WD. Symbols

832 individual mice, bars are median, unpaired $t$-test ${ }^{* *} p<0.005$.

833 f) Left: Fractions of 148M-huFRG mice with lobular inflammation on chow and 4 weeks on WD.

834 Number of mice with lobular inflammation per group at bottom of bars. Right: Lobular inflammation 835 score in the human graft of 148M-huFRG mice on chow and after 4 weeks of WD. Symbols 836 individual mice, bars are median, unpaired $t$-test.

837 g) Volcano plots showing differently expressed transcripts mapped to human reference genome 838 (human reads) from 148M-huFRG versus 148I-huFNRG livers on chow (left) and after 4 weeks 839 on WD (right). Red symbols FDR $<0.05$, grey denotes not significant.

841 Figure 6: PNPLA3-148M overexpression in hepatocytes exacerbates steatosis in mice on 842 WD

843 a) Experimental timeline of creating PNPLA3 overexpressing mice and diet challenge. Mouse 844 passaged primary human hepatocytes $(\mathrm{PHH})$ cultures were transduced with lentiviral vectors 845 expressing PNPLA3 variants and re-transplanted. Following humanization, huFNRG with 846 PNPLA3 transduced hepatocytes (td) were exposed to 4 weeks of WD.

847 b) H\&E staining on livers from tdRFP, td148I and td148M mice after 4 weeks on WD.

c) Liver steatosis score of the human graft in tdRFP, td148I and td148M mice on chow and after 8494 weeks on WD. Symbols individual mice, bars are median, unpaired $t$-test with ${ }^{* *} p<0.005$, $850 \quad{ }^{* * *} p<0.0005$ and ${ }^{* * *} p<0.0001$

851 d) Hepatic triglyceride quantification in tdRFP, td148I and td148M mice on chow and after 4 weeks 852 on WD. Symbols individual mice, bars are median, unpaired $t$-test, ${ }^{*} p<0.05$.

853 e) NAFLD Activity Score (NAS) in the human graft of tdRFP, td148I and td148M mice on chow 854 and after 4 weeks on WD. Symbols individual mice, bars are median, unpaired $t$-test, * $p<0.05$, $855 \quad{ }^{* *} \mathrm{p}<0.005,{ }^{* * *} \mathrm{p}<0.0005$ 
856 f) Volcano plot of 80 human genes expressed in livers of td148I versus td148M mice after 4 weeks

857 on WD. Expression by qRT-PCR normalized to 11 housekeeping genes, $\mathrm{n}=3-4$ mice per group, 858 burgundy symbols $p<0.05$, grey symbols not statistically significant.

860 Suppl Figure S6: PNPLA3-148M overexpression in hepatocytes exacerbates steatosis in 861 mice on WD

862 a) Serum human albumin (hAlb) levels in FNRG mice after transplantation with transduced PHH. 863 Symbols are mean \pm SEM of 3-16 mice.

864 b) human PNPLA3 mRNA levels in livers of tdRFP, td148I and td148M mice on chow and 4 weeks 865 on WD. Expression by qRT-PCR normalized to 11 housekeeping genes. Symbols individual mice, bars are median, unpaired $t$-test, ${ }^{* *} \mathrm{p}<0.005,{ }^{* * *} \mathrm{p}<0.0005$ and ${ }^{* * *} \mathrm{p}<0.0001$

c) H\&E staining on livers from tdRFP, td148I and td148M mice on chow. White arrow indicates macrovesicular and black arrow microvesicular steatosis.

869 d) Left: Fractions of tdRFP, tdr148I and td148M mice with human hepatocyte ballooning 870 degeneration on chow and 4 weeks on WD. Number of mice with ballooning per group at bottom 871 of bars. Right: Ballooning score in the human graft on chow and after 4 weeks of WD. Symbols 872 individual mice, bars are median, unpaired $t$-test ${ }^{* *} \mathrm{p}<0.005$ and ${ }^{* \star * *} \mathrm{p}<0.0001$

873 e) Left: Fractions of tdRFP, tdr148I and td148M mice with lobular inflammation on chow and 4 874 weeks on WD. Number of mice with lobular inflammation per group at bottom of bars. Right: 875 Lobular inflammation score in the human graft on chow and after 4 weeks of WD. Symbols 876 individual mice, bars are median, unpaired $t$-test.

877 f) Volcano plot of 80 human genes expressed in livers of td148I versus td148M mice on chow. 878 Expression by qRT-PCR, normalized to 11 housekeeping genes, $\mathrm{n}=3-4$ mice per group, green 879 symbols $p<0.05$, grey symbols not statistically significant. 
881 Figure 7: A HFD causes more active steatohepatitis in PNPLA3-148M overexpressing mice

882 a) H\&E staining on livers from tdRFP, td148I and td148M mice after 4 weeks on HFD.

883 b) Liver steatosis score of the human graft in tdRFP, td148I and td148M mice on chow and after 8844 weeks on HFD. Symbols individual mice, bars are median, unpaired $t$-test with ${ }^{* *} p<0.005$.

885 c) H\&E staining on liver from a td148M mouse after 4 weeks of HFD. White arrow indicates lobular 886 inflammation, black arrow microvesicular steatosis and grey arrow Mallory-Denk body.

887 d) NAFLD Activity Score (NAS) in the human graft of tdRFP, td148I and td148M mice on chow 888 and after 4 weeks on HFD. Symbols individual mice, bars are median, unpaired $t$-test, ${ }^{*} p<0.05$.

889 e) Low and high magnification fluorescent images of livers from td148I and td148M mice after 4 890 weeks of HFD stained for neutral lipids (green, BODIPY) and nuclei (blue, DAPI).

891 f) Two-dimensional density plots of neutral lipids (BODIPY) and RFP of 355,161 small 892 hepatocytes in livers from four td148I mice and 183,289 small hepatocytes in livers from three 893 td148M mice after 4 weeks of HFD. Density plots are displaying RFP in X-, and BODIPY 894 intensities in Y-Axis, both in arbitrary units (AU). Dashed lines display cell population group 895 quadrants, with lower left quadrant harboring double negative and upper right quadrant harboring 896 double positive cells. Solid line is simple linear regression with equation displayed underneath, $897 \quad r^{2}$ is Pearson correlation coefficient.

899 Supplement Figure S7: A HFD causes more active steatohepatitis in PNPLA3-148M 900 overexpressing mice

901 a) Hepatic triglyceride quantification in td148I and td148M mice after 4 weeks on HFD. Symbols 902 individual mice, bars are median, unpaired $t$-test, ${ }^{*} \mathrm{p}<0.05$.

903 b) Left: Fractions of tdRFP, tdr148I and td148M mice with human hepatocyte ballooning 904 degeneration 4 weeks on HFD. Number of mice with ballooning per group at bottom of bars. 
905 Right: Ballooning score in the human graft and after 4 weeks of HFD. Symbols individual mice, 906 bars are median, unpaired $t$-test.

907 c) Left: Fractions of tdRFP, td148I and td148M mice with lobular inflammation 4 weeks on HFD. 908 Number of mice with lobular inflammation per group at bottom of bars. Right: Lobular inflammation 909 score in the human graft after 4 weeks of HFD. Symbols individual mice, bars are median, 910 unpaired $t$-test.

911 d) human PNPLA3 mRNA levels in livers of td148I and td148M mice 4 weeks on HFD. Expression 912 by qRT-PCR normalized to 11 housekeeping genes. Symbols individual mice, bars are median, 913 unpaired $t$-test

914 e) H\&E staining on liver of non-transduced huFNRG mouse with severe steatosis (left). Right

915 panel shows two-dimensional density plot of neutral lipids (BODIPY) and RFP of 54,249

916 small hepatocytes in liver from one mouse displayed on left. Densitograms are displaying RFP

917 in X-, and BODIPY intensities in Y-Axis, both in arbitrary units (AU). Lower left quadrant are

918 double negative, upper right quadrant are double positive cells. Number in corners display

919 fraction of total cell population.

921 TABLE LEGENDS

922 Table 1) qRT-PCR mRNA expression in transduced huFNRG animals - average fold change 923 over housekeeping

924 Shown are fold changes of the expression of 80 human genes over 11 housekeeping genes 925 (GAPDH, HPRT1, GUSB, ACTB, B2M, HMBS, IPO8, PGK1, RPLP0, TBP and TFRC) from livers 926 of tdRFP, td148I and td148M mice on chow or on WD. Expression by qRT-PCR, $n=3-4$ mice per 927 group. 


\section{ACKNOWLEDGEMENTS}

930 This work was supported by the National Institutes of Health Grants R37DK048873, 931 R01DK056626 and R01DK103046 (to DEC), R01DK085713 (to CMR), K08DK090576 and 932 R01AA027327 (to YPJ) and the Starr Foundation (to CMR). PM was supported by grants from 933 Ghent University (Special Research Fund - ICOH expert center) and the Research Foundation 934 Flanders (grants VirEOS 30981113 and G047417N). MK received funding from the Deutsche 935 Forschungsgemeinschaft under grant number KA4688/1-1. The project was co-sponsored by the 936 Center for Basic and Translational Research on Disorders of the Digestive System through the 937 generosity of the Leona M. and Harry B. Helmsley Charitable trust (to EM). The NYULH Center 938 for Biospecimen Research and Development, Histology and Immunohistochemistry Laboratory 939 (RRID:SCR_018304) is supported in part by the Laura and Isaac Perlmutter Cancer Center 940 Support Grant; NIH/NCI P30CA016087. The RU Center for Clinical and Translational Science 941 Bioinformatics program is supported by the Clinical and Translational Award (CTSA) and the 942 National Center for Advancing Translational Sciences (NCATS), part of the National Institutes of 943 Health. We thank Branka Brukner Dabovic (NYULH - Department of Cell Biology Research) for 944 help with imaging fluorescence samples. We thank the Rockefeller University High-Throughput, 945 Bioimaging and Genomics Resource Centers. 


\section{MATERIAL AND Methods}

\section{Mice}

950 Male and female Fah $^{-/-}$NOD Rag1 $1^{-/-}$II2rgnull $(F N R G)$ mice $^{24}$ were preconditioned with retrorsine ${ }^{25}$ 951 and male $\mathrm{Fah}^{-/-} \mathrm{Rag}^{-/-} \| 12 \mathrm{rg}^{-/-}$(FRG) mice ${ }^{26}$ with adenoviral urokinase plasminogen activator 952 (UPA) before transplantation with primary human hepatocytes (PHH). NOD Rag1 $1^{-/-} \| 2$ rgull $^{\text {nuRG}}$ (NR) 953 mice were obtained from Jackson Labs (Bar Harbor, ME) and received retrorsine and nitisinone 954 cycling similarly to FNRG mice. Seven week old male thymidine kinase transgenic mice on the 955 NOD SCID $/ 12 r^{\text {null }}$ background $\left(\right.$ TK-NOG) ${ }^{35}$ were obtained from Taconic Biosciences 956 (Germantown, NY) and preconditioned with retrorsine ${ }^{25}$ and 48 hours $0.1 \mathrm{mg} / \mathrm{ml}$ valganciclovir 957 (Sigma) in drinking water 14 days prior to transplantation. Male transgenic $\mathrm{UPA}^{+/ /} / \mathrm{SCID}$ mice were 958 transplanted as previously described. ${ }^{36}$ The following $\mathrm{PHH}$ donors were used: cryopreserved or 959 mouse-passaged (mp)PHH1 (HUM4188, Lonza), cryopreserved or mpPHH2 (HUM4129, Lonza), 960 cryopreserved PHH3 (HFCP940, BD Biosciences) and cryopreserved PHH4 (HHF13022, 961 Yecuris). Hepatocytes were genotyped only for the PNPLA3 rs734809 variant. Genotyping was 962 performed by Sanger sequencing using forward 5'-GCCCTGCTCACTTGGAGAAA-3' and 963 reverse primer 5'-TGAAAGGCAGTGAGGCATGG-3' as reported. ${ }^{70}$ To make PNPLA3 964 overexpressing mice, PNPLA3-148I or $-148 \mathrm{M}$ (both containing the K434E variant ${ }^{71}$ ) were cloned 965 into pSCRPSY69 (GenBank: KT368137.1) to generate VSVg pseudotyped lentiviral particles. ${ }^{25}$

966 Driven by HIV-LTR promoter, pSCRPSY allows a gene of interest (in our case either PNPLA3 967 variants or none in "tdRFP" controls) to be expressed from the completely spliced "early" HIV-1 968 mRNA, while TagRFP (a surrogate marker for transduction) is expressed from an unspliced late HIV9691 mRNA. ${ }^{45}$ mpPHH1 were isolated from huFNRG, plated in 6 well collagen-coated plates and 970 transduced with pSCRPSY vectors by $1000 \mathrm{~g}$ spinoculation. Three to 5 days after transduction $971 \sim 5 \times 10^{5}$ cells/mouse were retransplanted into retrorsine preconditioned FNRG animals as 972 described..$^{25}$ Mice were housed in a 12-hour light/dark cycle at $21^{\circ} \mathrm{C}$ and $50 \%$ humidity. All 
973 experiments were conducted under animal use protocols approved by Rockefeller University,

974 Ghent University and Yecuris corporation. huFNRG and huTK-NOG mice were humanized at 975 Rockefeller University, huFRG mice at Yecuris corporation and hu-uPA/SCID mice at Ghent 976 University.

\section{Diet challenges}

979 One week prior and during dietary studies, huFNRG, huFRG, NRG and muFNRG mice were 980 changed from oral nitisinone (CuRx, Yecuris cat\#20-0028) to intraperitoneal (i.p.) injections at $9811 \mathrm{mg} / \mathrm{kg}$ body weight for three days every 2 weeks. Injection solution was $0.5 \mathrm{mg} / \mathrm{ml}$ nitisinone 982 dissolved in 500mM NaHCO $3 \mathrm{pH} 7.4$ and diluted 1:10 in PBS-/- (Gibco, Thermo Fisher) to final 983 concentration of $50 \mu \mathrm{g} / \mathrm{ml}$. During humanization or 'murinization' the FNRG, FRG and TK-NOG 984 lines were maintained on an amoxicillin containing chow diet (modified PicoLab Mouse Diet 20, 9855058 - 5B1Q, 0.12\% Amoxicillin, irradiated; TestDiet, Richmond, IN) and autoclaved water. One 986 week prior dietary challenge, amoxicillin medicated chow was replaced with regular chow diet 987 (PicoLab Rodent Diet 20, 5053, irradiated; LabDiet, Fort Worth, TX) lacking amoxicillin. For High 988 Fat Diet (HFD) studies, mice were given a diet with $60 \%$ kcal fat, $20 \%$ carbohydrates and $20 \%$ 989 protein (D12492i irradiated; Research Diets, New Brunswick, NJ) and autoclaved water. For 990 Western-style Diet (WD) studies, HFD was combined with $10 \% \mathrm{w} / \mathrm{v}$ sucrose (Fisher Scientific 991 cat\#S5-3; Fair Lawn, NJ) in autoclaved water. Sucrose drinking water was freshly made once a 992 week. UPA $^{+/+} /$SCID mice were fed irradiated standard breeding chow (Mouse Breeding complete 993 feed for mice (GE 16.7MJ/kg; 14.0 MJ/kg), Ssniff Spezialdiäten GmbH, Soest, Germany). All diets 994 and drinking water were given ad libitum. Diet challenges in huFNRG, huFRG, NRG, muFNRG 995 and huTK-NOG mice were done at Rockefeller University. 
998 Serum was obtained from tail veins, retroorbital or submental venipuncture. Human albumin was

999 quantified as previously described ${ }^{24}$ and serum mouse insulin quantified using Ultra-Sensitive 1000 Mouse Insulin ELISA Kit (Crystal Chem cat\#90080, Elk Groove Village, IL) following 1001 manufacturer's instructions. In mice with hAlb serum levels $>8 \mathrm{mg} / \mathrm{ml}$, ALT and AST activity 1002 (Elabscience cat\#E-BC-K235 and K236, China), which is capable to detect mouse activity as per 1003 manufacturer, and human ALT protein (Abcam cat\#ab234578) were determined following 1004 manufacturers instruction. Serum was obtained from submental venipuncture 2 to 3 days after 1005 last nitisinone injection cycle to minimize mouse transaminase activity. Tolerance tests to glucose 1006 (GTT), insulin (ITT) and pyruvate (PTT) were performed in FNRG mice in the weeks with no NTBC 1007 cycling injections similar as described..$^{72}$ Briefly, age matched mice 4 to 12 weeks after dietary 1008 challenge with WD or chow were fasted with free access to water for $6 \mathrm{~h}$ prior to GTT and ITT 1009 and for $16 \mathrm{~h}$ prior to PTT. Approximately $5 \mu \mathrm{l}$ blood was collected from tail bleed before and at 1010 indicated intervals for up to $180 \mathrm{~min}$ following i.p. injection with $2.5 \mathrm{~g} / \mathrm{kg}$ body weight glucose 1011 (Sigma Aldrich cat\#G7021), $1 \mathrm{U} / \mathrm{kg}$ body weight human insulin analogue (Eli Lilly, Humulin R U1012 100), or $2 \mathrm{~g} / \mathrm{kg}$ body weight pyruvate (Sigma Aldrich cat\#P2256), each dissolved in isotonic PBS1013 /- (Gibco, Thermo Fisher Scientific). Blood glucose concentrations were determined using a 1014 GE100 Blood Glucose Monitor (GE, Ontario, CA). Prior to terminal blood and tissue collection (2 1015 to 3 days after last nitisinone injection in $\mathrm{Fah}^{-/}$strains), mice were fasted for 6 hours during light 1016 cycle with access to water. Blood was collected by submental and/or retroorbital venipuncture 1017 and tissue samples were weighed and stored in fixative or frozen on dry-ice and stored at $-80^{\circ} \mathrm{C}$.

1018 Blood was centrifuged with $16,000 \times g$ for $15 \mathrm{~min}$ at $4^{\circ} \mathrm{C}$ and plasma was collected and stored at $1019-80^{\circ} \mathrm{C}$. Hepatic lipids were extracted from frozen liver tissue as described ${ }^{72}$ and liver and plasma 1020 TG, NEFA, total and free cholesterol, and phospholipids were quantified according to 1021 manufacturer's instructions (Wako Diagnostics, Mountain View, CA). Equal volumes of plasma 1022 were pooled from three mice and lipoproteins were fractionated by fast protein liquid 
1023 chromatography (ÄKTA pure FPLC system, GE Healthcare, Pittsburgh, PA, USA) and triglyceride 1024 and cholesterol in fractions quantified with reagent kits (Wako Diagnostics, Mountain View, CA). ${ }^{73 \text {, }}$

\section{Liver histology and quantification}

1028 Neutral buffered formalin-fixed (Thermo Fisher Scientific cat\#5725) paraffin-embedded liver samples were sectioned at 5 $\mu \mathrm{m}$ onto charged slides (Fisher Scientific cat\#22-042-924). Slides were dried for 1 hour at $60^{\circ} \mathrm{C}$, deparaffinized in xylene, rehydrated through a graded series of ethanol, and rinsed in distilled water for 5 minutes. Sections were then stained with Hematoxylin

1032 (Richard-Allan Scientific cat \#7211, USA) and Eosin (Leica cat\#3801619) using our routine laboratory method described in [Carson, F.L., Histotechnology: a self instructional text. 2009, Chicago: ASCP Press., 114-123], and with Picro Sirius Red Stain (PSR) (Abcam cat\#ab150681) and Masson's Trichrome (Newcomer Supply cat\#9179A, USA) in accordance with manufacturer's supplied procedures. For chromogenic immunohistochemistry staining, unconjugated, polyclonal rabbit anti-Human Nuclear Mitotic Apparatus protein (Abcam Cat\# 97585 Lot\# GR268490 RRID AB_10680001), ${ }^{27}$ and unconjugated, mouse anti-Human Glutamine Synthetase (Ventana Medical Systems Lot\# V0001337 RRID AB_2861318) clone GS-9 ${ }^{75}$ were used. ${ }^{75}$ Slides were scanned using a Leica Aperio AT2 System and digitally archived via eSlide Manager. To preserve lipids with and without fluorescent proteins for histopathological analysis, fresh liver samples

1042 haven been fixed in 4\% paraformaldehyde (Electron Microscopy Sciences) for 18 hours, 1043 cryoprotected at $4 \stackrel{\circ}{\circ}$ with $10 \%$ and $18 \%$ sucrose $w / v$ for 24 hours each before OCT (Sakura 1044 Finetek) embedding and storage at $-80^{\circ} \mathrm{C}$. OCT-embedded frozen specimens were cryosectioned 1045 at $5 \mu \mathrm{m}$ onto charged slides (Fisher Scientific cat\# 22-042-924). Slides were air-dried at room 1046 temperature overnight and fixed in 10\% neutral buffered formalin for 1 hour at room temperature. 1047 Slides were then rinsed in distilled water, stained with BODIPY (Invitrogen cat\# D3922) and Oil 
1048 Red O (Sigma-Aldrich cat\# O1391). For BODIPY staining, slides were incubated in Reaction 1049 Buffer (Ventana cat\# 950-300) for 10 minutes and stained with BODIPY (1:1000 dilution) and DAPI (1:1000 dilution) in PBS for 60 minutes, and covered using ProLong ${ }^{\text {TM }}$ Diamond Antifade 1051 Mountant (Invitrogen, cat\# P36961). BODIPY, RFP, and DAPI were imaged on a Hamamatsu 1052 Nanozoomer 2.0 HT and digitally archived via SlidePath. For Oil Red O staining, sections were 1053 stained in Oil Red $O$ solution for 10 minutes, rinsed with distilled water for 5 minutes, 1054 counterstained with hematoxylin (Richard-Allan Scientific cat\# 7211,) for 1 minute and rinsed in distilled water for 1 minute. Samples were blued in $1.2 \%$ ammonium hydroxide solution for 1 minute, rinsed in distilled water for 1 minute, and coverslipped using a $50 \%$ glycerol solution. Slides were scanned using a Leica Aperio AT2 System and digitally archived via eSlide Manager. 1058 For fluorescence image analyses SVS image files derived from Hamamatsu Nanozoomer 2.0 HT were extracted and saved as uncompressed multiple 40x magnification tile images in TIFF format using FIJI (https://imagej.net/Fiji/Downloads) ${ }^{76}$ and NDPITools plugin. ${ }^{77}$ TIFF files of tile images were split into fluorescence channels (DAPI, BODIPY or RFP) and saved as 8-bit grayscale images. Masks were generated around human hepatocytes nuclei approximated by diameter size in DAPI images, enlarged three folds to approximate the size of human hepatocytes in chimeric livers and used for measuring BODIPY and RFP signal intensities. RFP and BODIPY intensities per approximated human hepatocyte were plotted as two-dimensional density plots using Python. Histological scores were conducted from H\&E stained samples by clinical pathologist (CF and $1067 \mathrm{MP})$, using the NAS system from NASH-CRN. ${ }^{43}$ Steatosis grade $(0:<5 \%, 1: 5-33 \%, 2:>33-66 \%$ 1068 and 3:> 66\%), lobular inflammation (0: no foci, 1: <2 foci / 200x field, 2: 2-4 foci, 3: $>4$ foci) and 1069 ballooning degeneration (0: none, 1: few ballooning cells, 2: many cells / prominent ballooning) were used to generate an overall score (NAS). Fibrosis staging was evaluated separately in PSR stained samples using the same system, ${ }^{43}$ with the following scores 0 : none, 1 : perisinusoidal or 
1072 periportal, 1A: mild, zone 3, perisinusoidal, 1B: moderate, zone 3, perisinusoidal, 1C: portal/periportal.

\section{Liver tissue transcriptional analyses}

Around $25 \mathrm{mg}$ of freshly harvested liver tissue was placed into $1 \mathrm{ml} 4^{\circ} \mathrm{C}$ TRIzol (Life Technologies, USA) with $500 \mu \mathrm{l}$ of $1 \mathrm{~mm}$ glass beads (BioSpec Products cat\# 11079110) and homogenized using MagNA lyser (Roche, USA), weighed and stored at $-80^{\circ} \mathrm{C}$. For RNA extraction, $500 \mu \mathrm{l}$ of liver/Trizol mixture was used for chloroform extraction using MaXtract ${ }^{\mathrm{TM}}$ (Qiagen, cat\# 129056) and RNeasy® Mini Kit (Qiagen, cat\# 74104) coupled with on-column DNasel treatment (Qiagen cat\#79254) following manufacturer's instructions. Extracted RNA was stored at $-80^{\circ} \mathrm{C}$. For qRTPCR, cDNA was generated from liver RNA using SuperScript IV VILO (Thermo Fisher Scientific cat\#11766050) following manufacturer's instructions. qRT-PCR was performed in 96-well Human Fatty Liver Array plates (Thermo Fisher Scientific cat \# 4391524) on a QuantStudio 3 System (Applied Biosystem) for the following genes: ABCA1, ACACA, ACADL, ACLY, ACOX1, ACSL5, ACSM3, ADIPOR1, ADIPOR2, AKT1, APOA1, APOB, APOC3, APOE, ATP5C1, CASP3, CD36, CEBPB;CEBPB-AS1, CNBP, CPT1A, CPT2, CYP2E1, CYP7A1, DGAT2, FABP1, FABP3, FABP5, FAS, FASN, FOXA2, FOX01, G6PC, G6PD, GCK, GK, GSK3B, HMGCR, HNF4A, IGF1, IGFBP1, IL1B, INSR, IRS1, LDLR, LEPR, LPL, MAPK1, MAPK8, MLXIPL, MTOR, NDUFB6, NFKB1, NR1H2, NR1H3, NR1H4, PCK2, PDK4, PIK3CA, PIK3R1, PKLR, PNPLA3, PPA1, PPARA, PPARG, PPARGC1A, PRKAA1, PTPN1, RBP4, RXRA, SCD, SERPINE1, SLC27A5, SLC2A1, SLC2A2, SLC2A4, SOCS3, SREBF1, SREBF2, STAT3, XBP1. Transcriptional changes were calculated over mean of housekeeping genes: GAPDH, HPRT1, GUSB, ACTB, B2M, HMBS, IPO8, PGK1, RPLPO, TBP, TFRC. Data were analyzed and graphed in R (version 4.0.2). For bulk RNA sequencing (RNAseq) the quantity and integrity of extracted liver RNA were assessed using an Agilent 2100 Bioanalyzer (Agilent Technologies, Palo Alto, CA). Library 
1097 construction and sequencing were performed by Novogene USA (Sacramento, CA), using 1098 Illumina S4 flowcell on Novaseq 6000 platform with a paired-end read length of $150 \mathrm{bp}$. 1099 Downstream RNAseq analysis was performed using a combination of programs. Alignments were 1100 parsed using STAR and differential expression was determined through limma/voom. ${ }^{78}$ 1101 Alignments were parsed using STAR and differential expression was determined through 1102 limma/voom. ${ }^{78} \mathrm{GO}, \mathrm{KEGG}, \mathrm{REAC}$ and TF enrichment were determined with the g:profiler $\mathrm{R}$ package. ${ }^{79}$ Reference genome and gene model annotation files were downloaded from genome website browser (NCBI/UCSC/Ensembl) directly. Indices of the reference genome were built using STAR ${ }^{80}$ and paired-end clean reads were aligned to human or mouse reference genome using STAR (v2.5), setting the outFilterMismatchNmax argument to 2, from which gene level count tables were built using HTseq-count. ${ }^{81}$ Gene-level read counts were then processed using the limma suite of tools ${ }^{78}$ first with a voom with quality weights transformation, followed by linear model fitting to determine differentially expressed genes. The $P$ values for individual genes were adjusted using the Benjamini \& Hochberg method. Corrected P-value of 0.05 and absolute foldchange of 1 were set as the threshold for significantly differential expression. Geneset testing on the entire dataset was performed using the camera() function to determine geneset direction using genesets extracted from MsigDB. ${ }^{82}$ Select genesets were plotted as dotplots from the camera output. Gene Ontology (GO), Kyoto Encylopedia of Genes and Genomes (KEGG), and REACTOME database enrichment analysis of differentially expressed genes was further implemented with the g:profiler R package,${ }^{79}$ in which gene length bias was corrected. Terms with corrected P-values less than 0.05 were considered significant. The high-throughput sequencing data from this study have been submitted to the NCBI Sequence Read Archive (SRA) under accession number \#\#\#\#\#\#. 
1122 Unless stated otherwise, statistical analyses were performed in GraphPad Prism (San Diego, Ca).

1123 Statistics are indicated in graphs if $p<0.05$.

\section{AUTHOR CONTRIBUTIONS:}

1126 MK, EM, SS, BE, CMR and YPJ designed research; MK, EM, SS, CGF, JML, JLP, MT, BR, IRL, 1127 C.Z., BZ, AFS, CQ, LF, AWA, WMS, SB, GL, LC and YPJ performed experiments; MK, EM, SS, 1128 CGF, JML, JLP, MT, BR, IRL, CZ, BZ, AFS, CQ, LF, AWA, YL, DEC, MP, LD, MG, PM, BAE,

1129 CMR and YPJ analyzed data; HS, NDT and RC provided new reagents/analytic tools; DEC, PM, 1130 CMR and YPJ secured funding; MK, EM, and YPJ wrote the paper; YPJ directed the study. 
Figure 1: Human hepatocytes in chimeric mice on WD rapidly develop steatosis

a
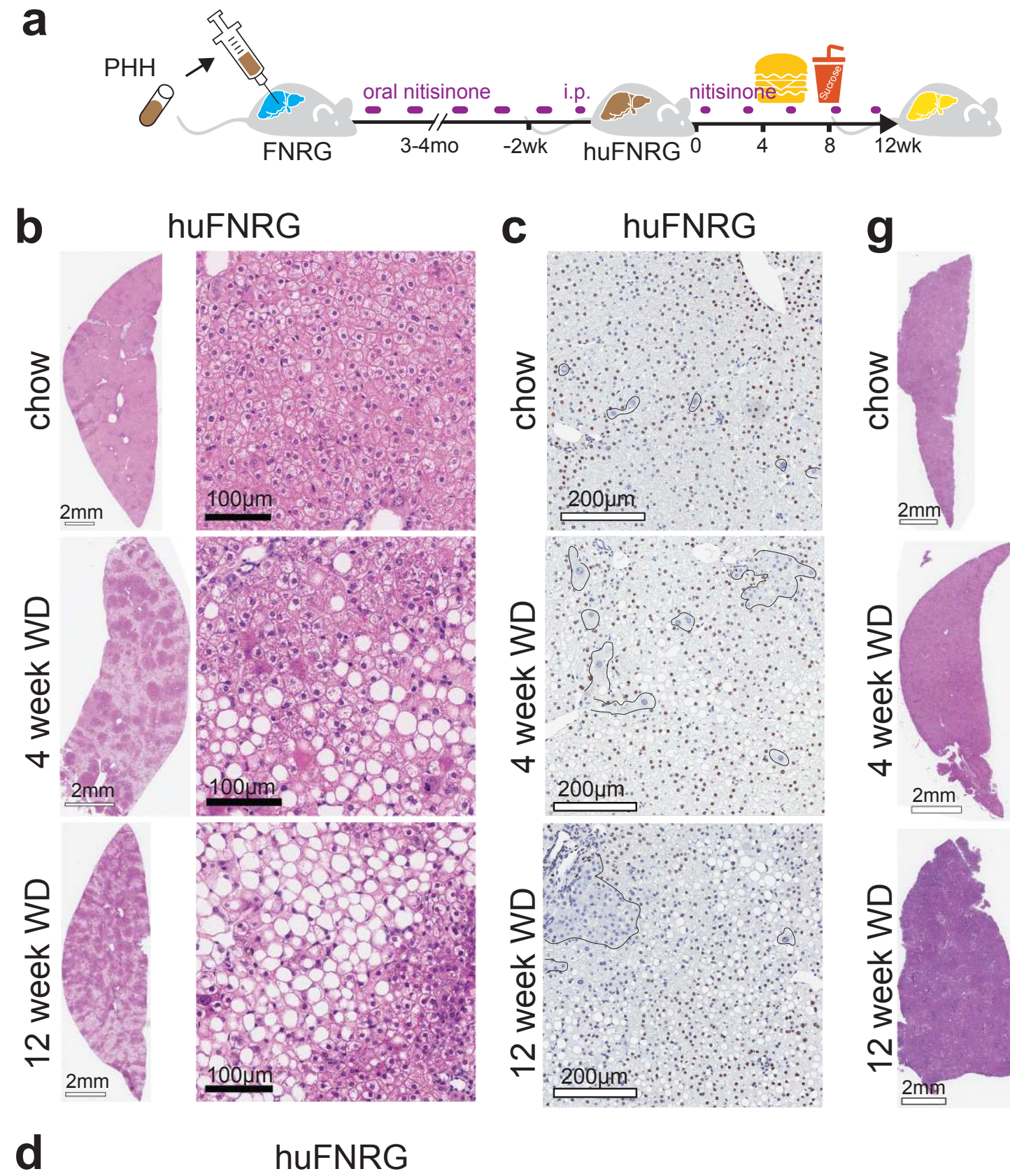
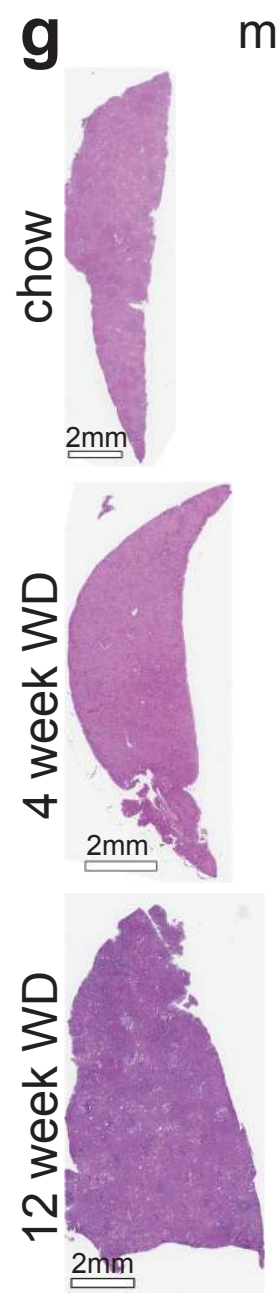

muFNRG
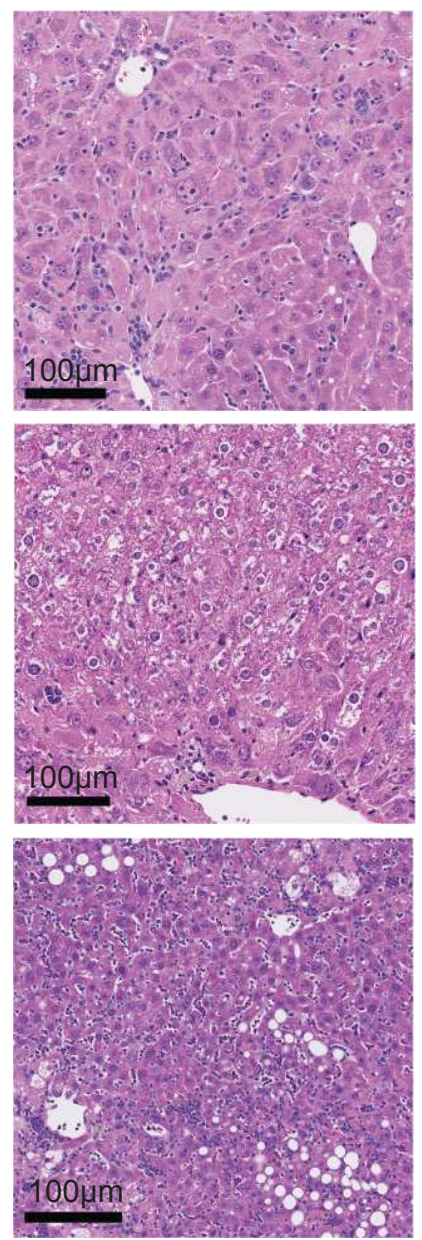

d

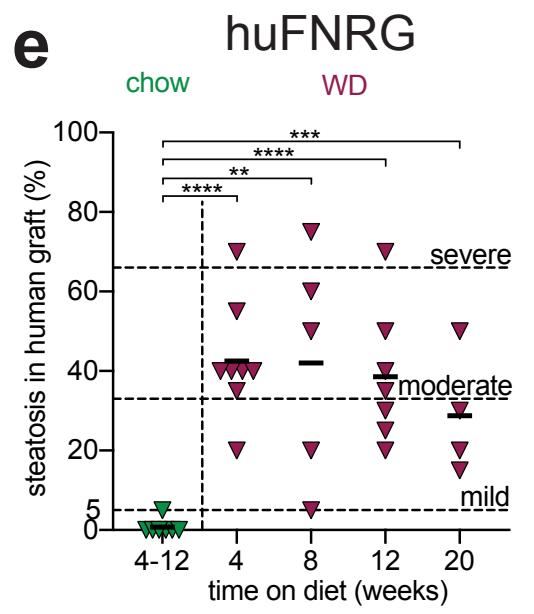

f huFNRG
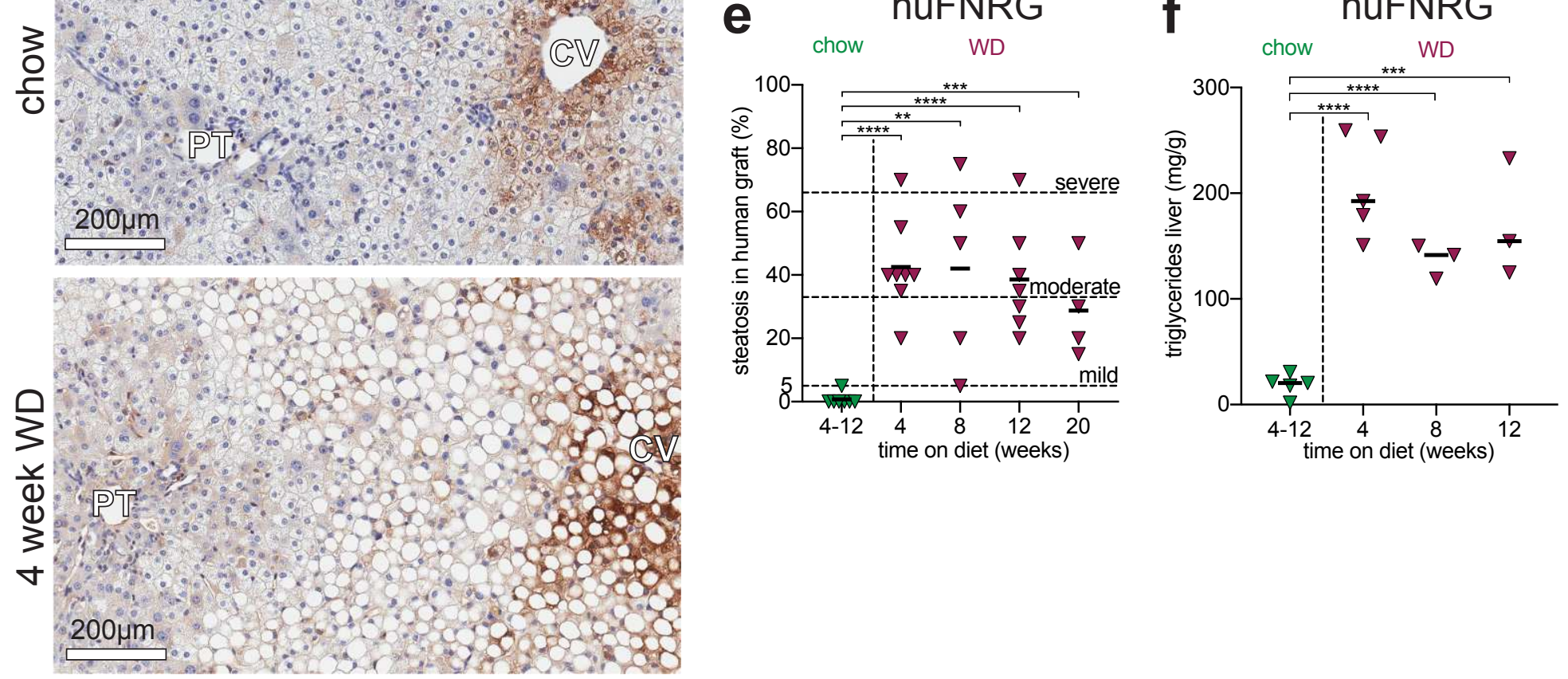
Figure 2: Systemic metabolic effects of WD in chimeric FNRG mice

a

huFNRG weight

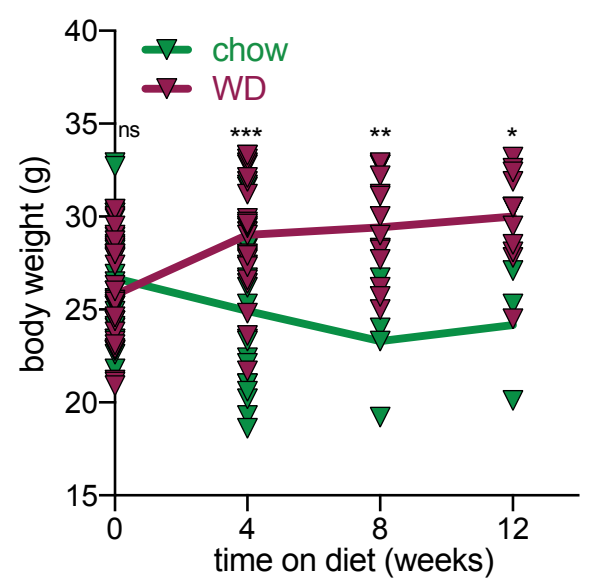

C

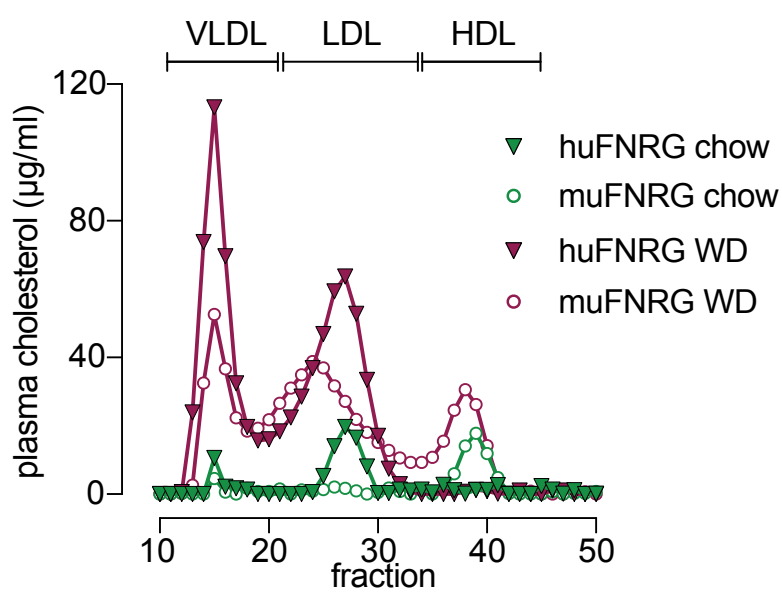

e

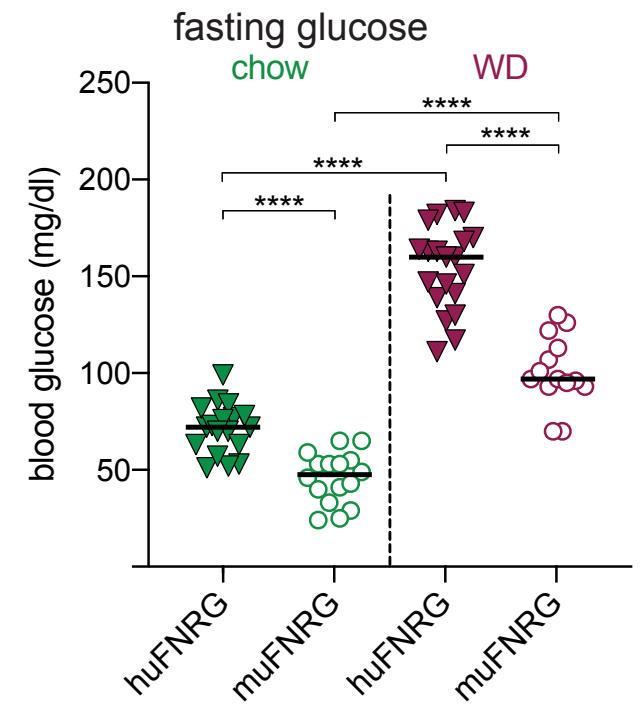

b

gonadal fat huFNRG

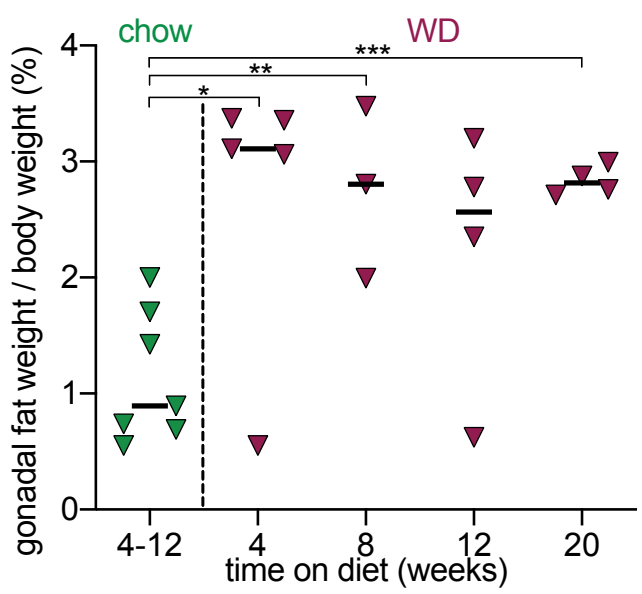

d

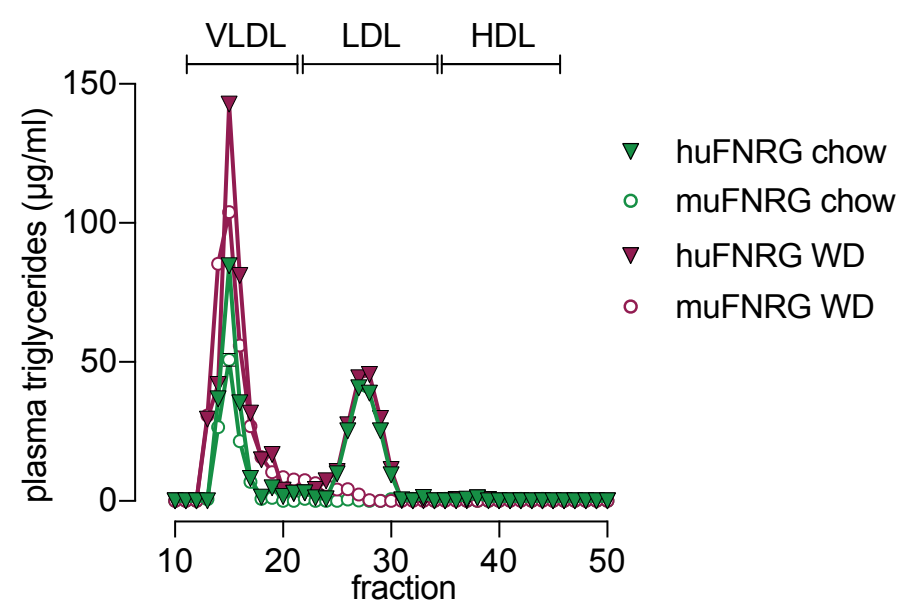

f

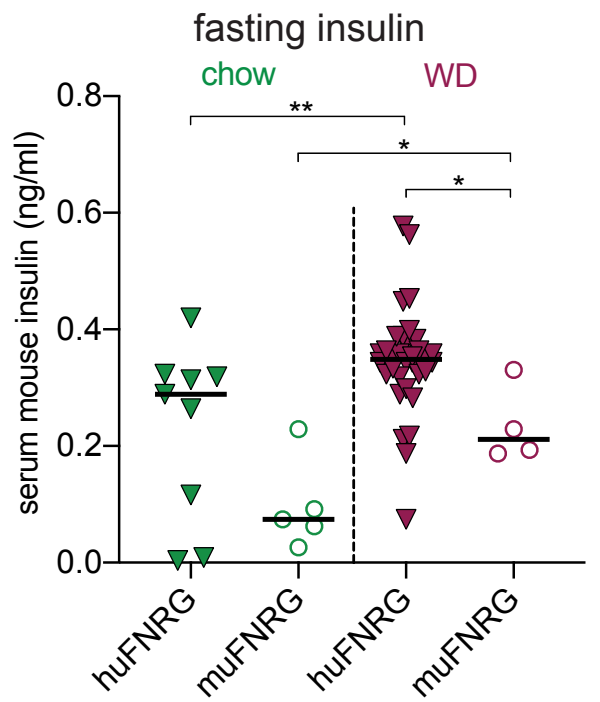

g

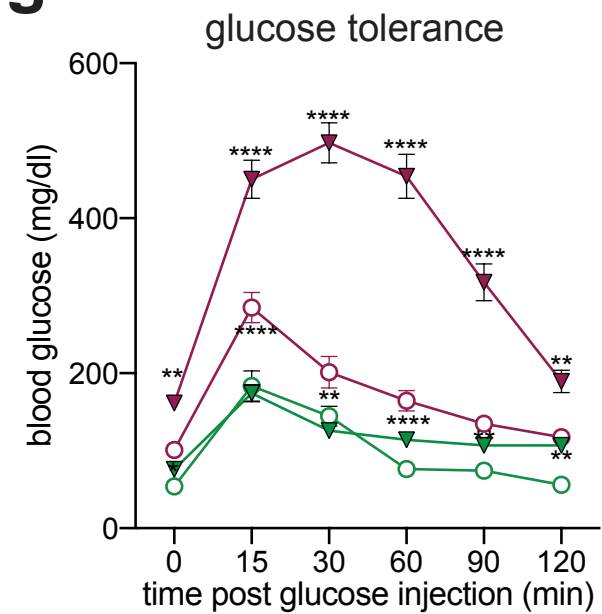

insulin tolerance

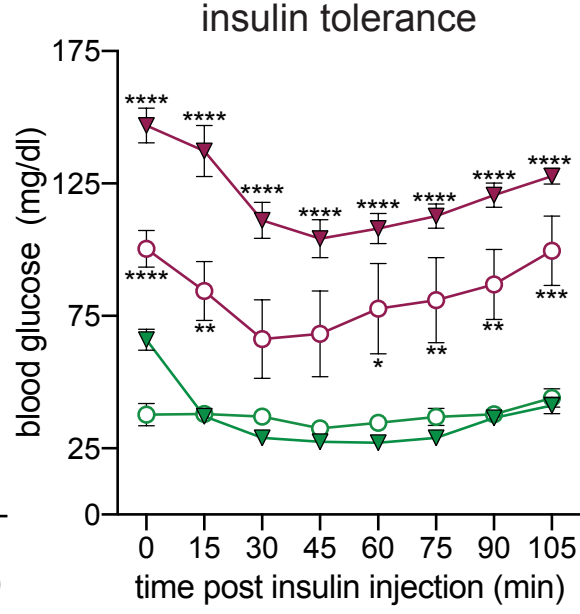

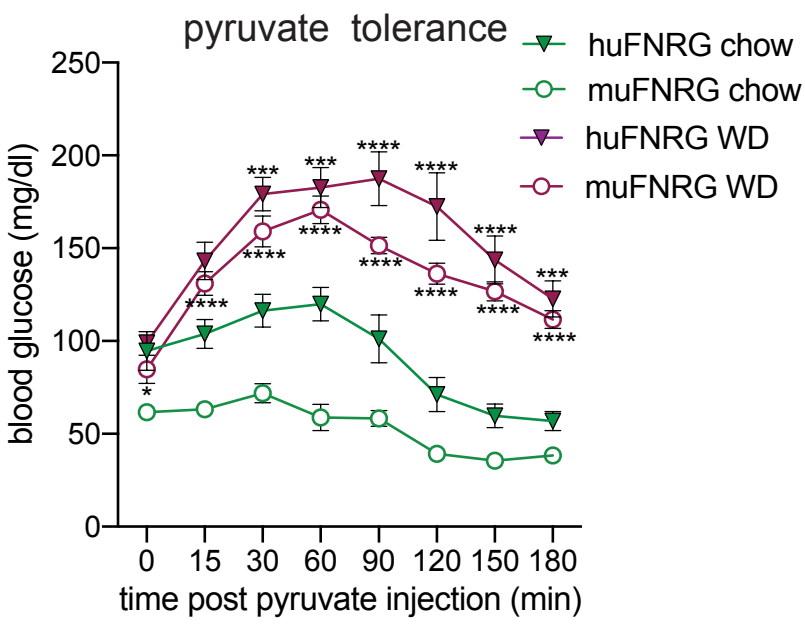


Figure 3: huFNRG mice on WD develop mild steatohepatitis
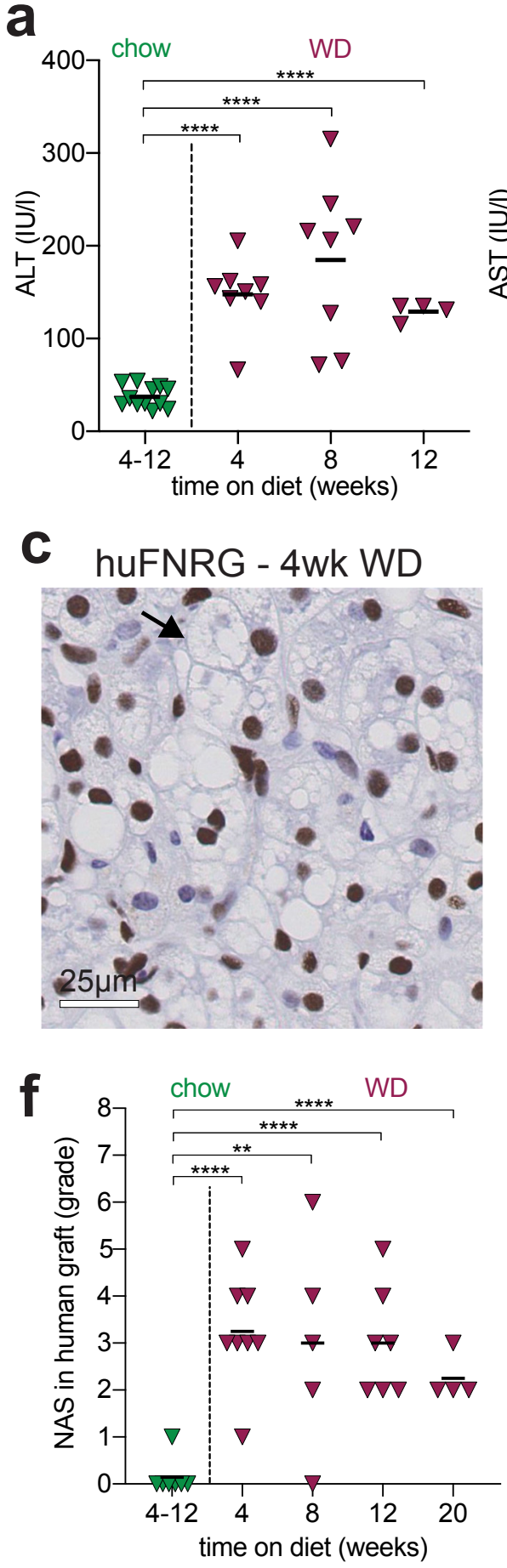

b huFNRG - 8wk WD

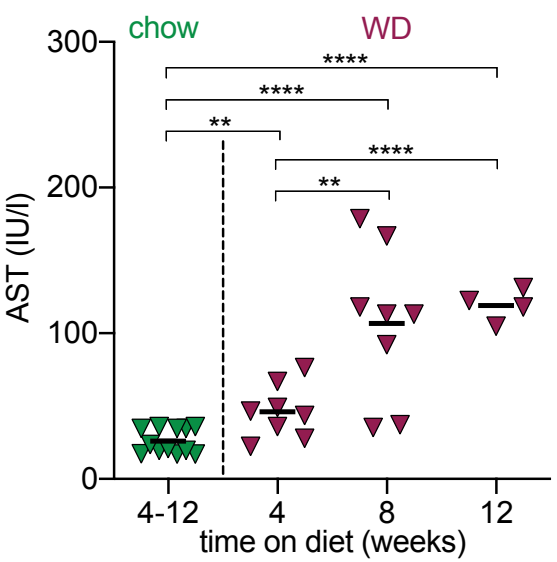

d

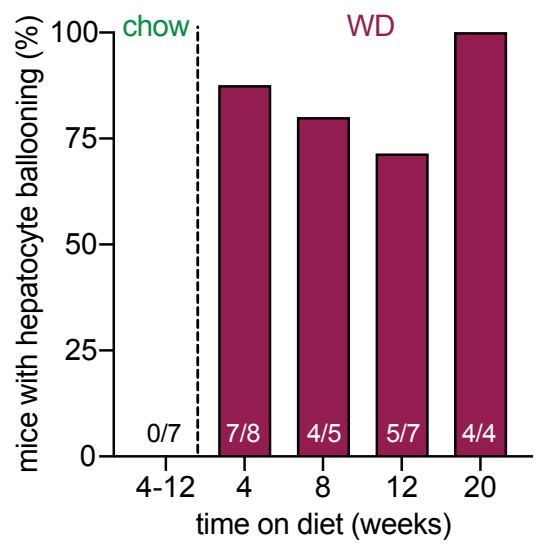

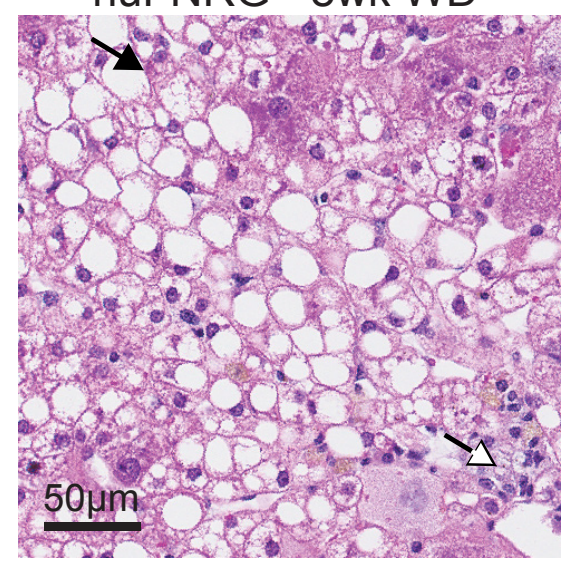

e

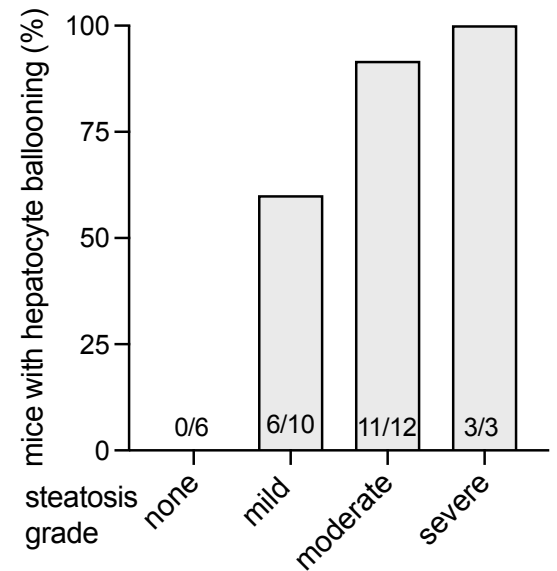

h

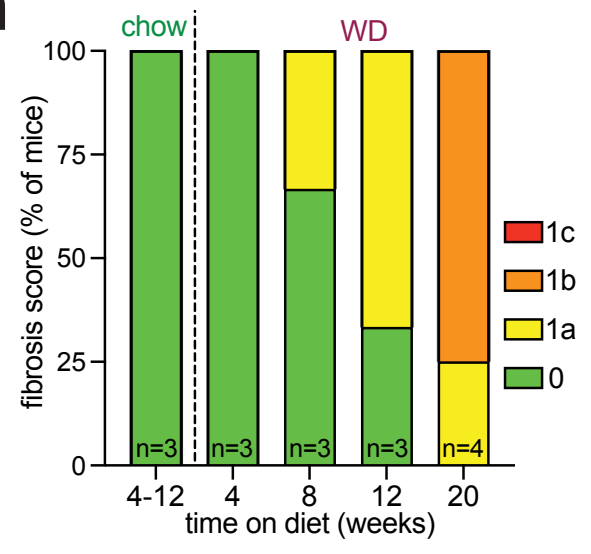

g 4 weeks WD 8 weeks WD 12 weeks WD 20 weeks WD

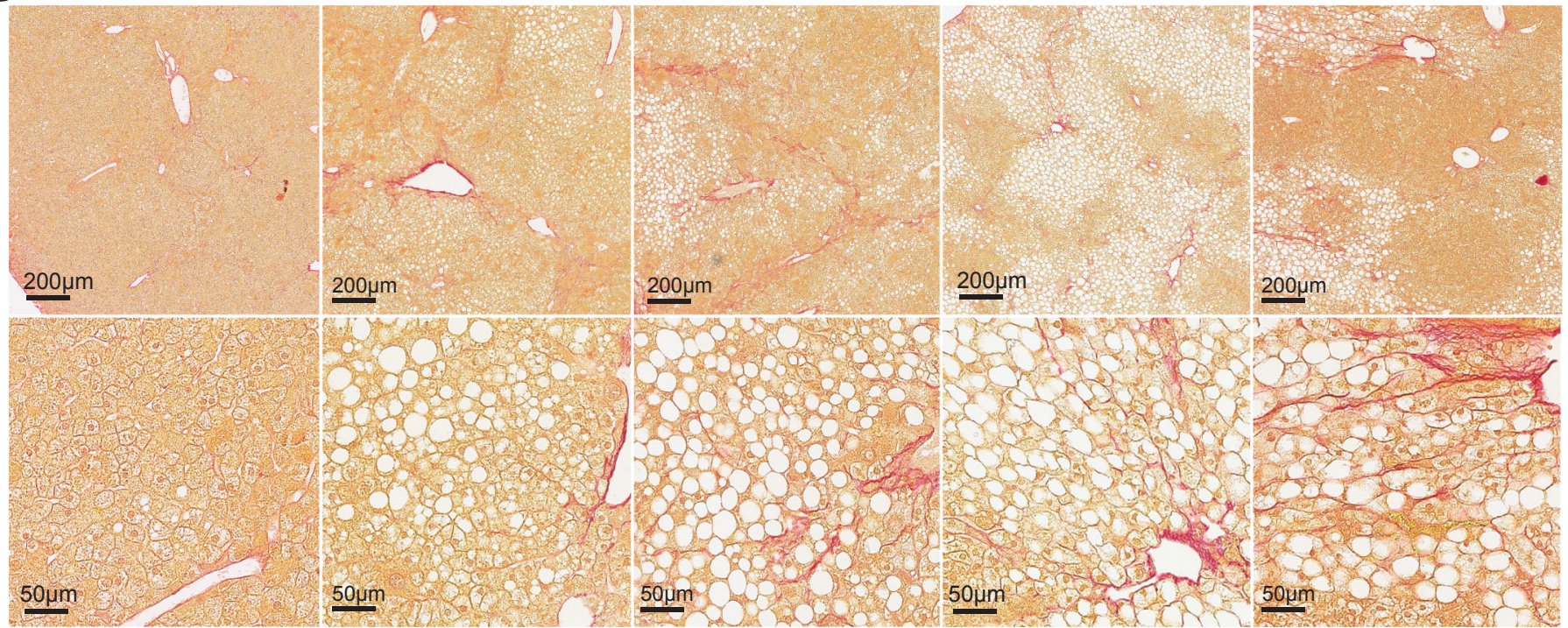




\section{Figure 4: Transcriptional changes in huFNRG mice on Western Diet}
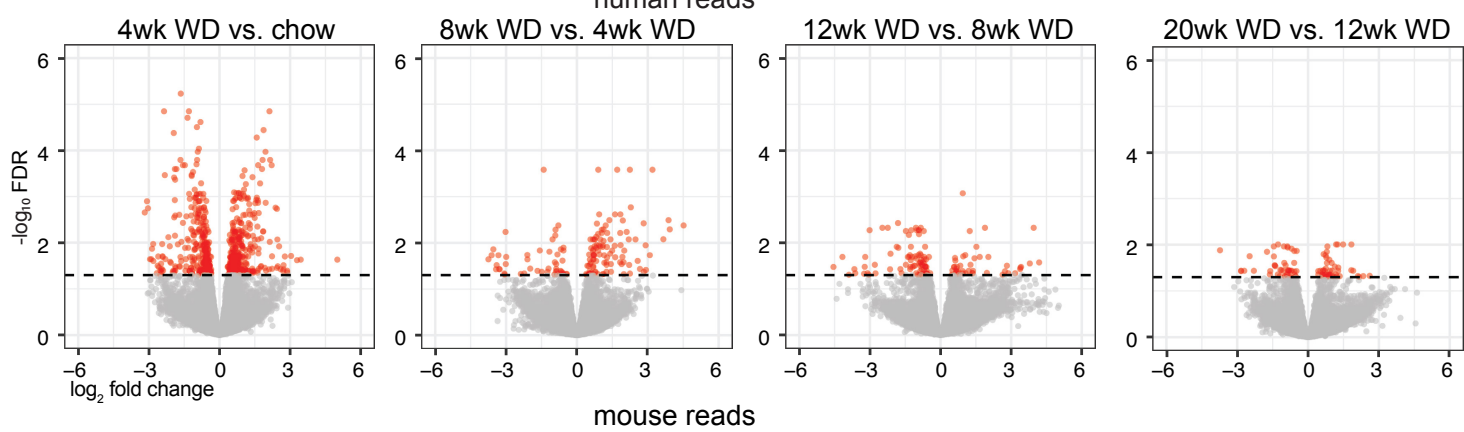

b

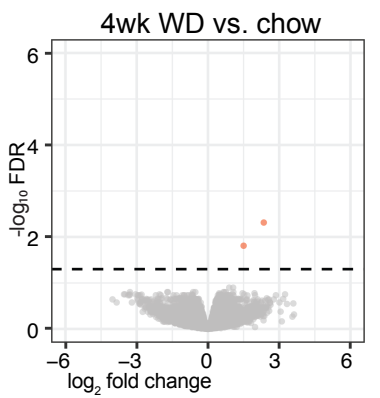

$8 w k$ WD vs. 4 wk WD
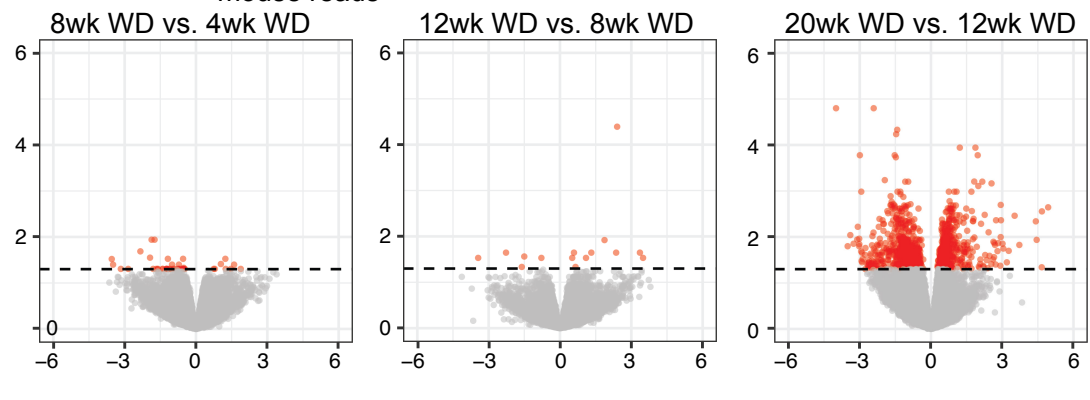

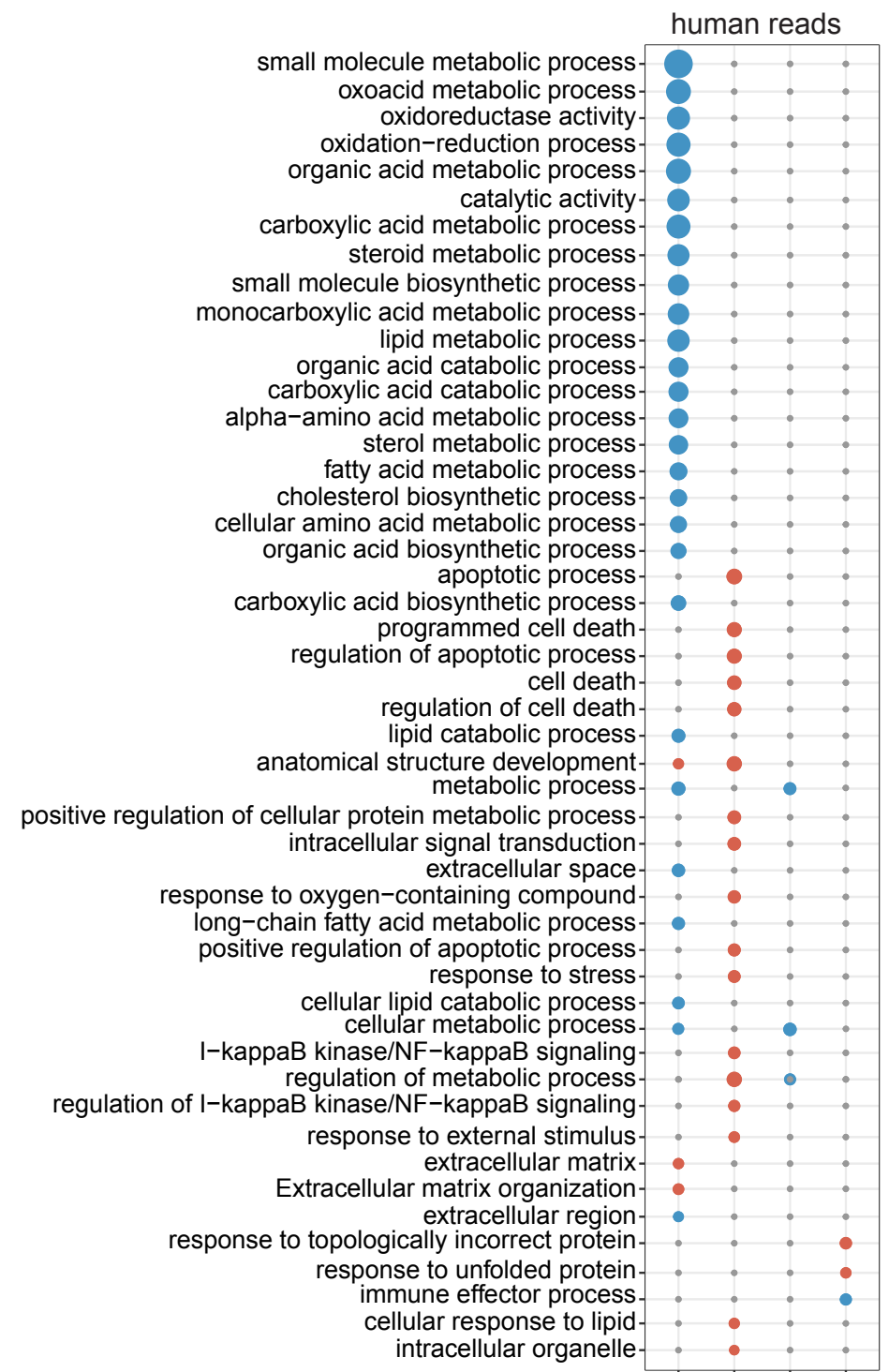

\begin{tabular}{|c|c|}
\hline $\begin{array}{l}-\log _{10}(p-a d j) \\
: \quad 0.25 \\
: \quad 0.50 \\
: \quad 2.50 \\
: 10.00 \\
: 20.00 \\
\bullet \quad 20.00\end{array}$ & $\begin{array}{l}\text { Direction } \\
\text { - Down } \\
\text { Not Significant } \\
\text { - Up }\end{array}$ \\
\hline
\end{tabular}

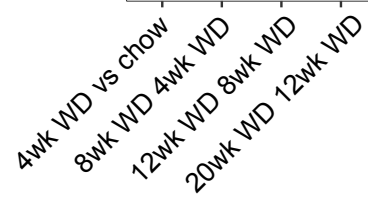

d

mouse reads

extracellular matrix cellular matrix cell migration cell adhesion extracellular matrix structural constituent biological adhesion regulation of cell migration vasculature development organic acid metabolic process carboxylic acid metabolic process blood vessel development oxoacid metabolic process anatomical structure development small molecule metabolic process developmental process anatomical structure development . catalytic activity

Extracellular matrix organization metabolic process

anatomical structure morphogenesis angiogenesis

intracellular membrane-bounded organelle

Steroid hormone biosynthesis oxidoreductase activity

cellular response to chemical stimulus Metabolic pathways intracellular organelle extracellular region

intracellular signal transduction alpha-amino acid metabolic process cellular amino acid metabolic process monocarboxylic acid metabolic process oxidation-reduction process response to wounding response to growth factor extracellular space wound healing

organic acid catabolic process carboxylic acid catabolic process

fatty acid metabolic process

collagen biosynthetic process response to stress

response to oxygen-containing compound small molecule biosynthetic process Retinol metabolism

organic acid biosynthetic process carboxylic acid biosynthetic process -

smooth muscle cell migration positive regulation of metabolic process $-\log _{10}(p-a d j)$ 
Figure 5: PNPLA3-148M huFRG mice on WD develop steatohepatitis

a
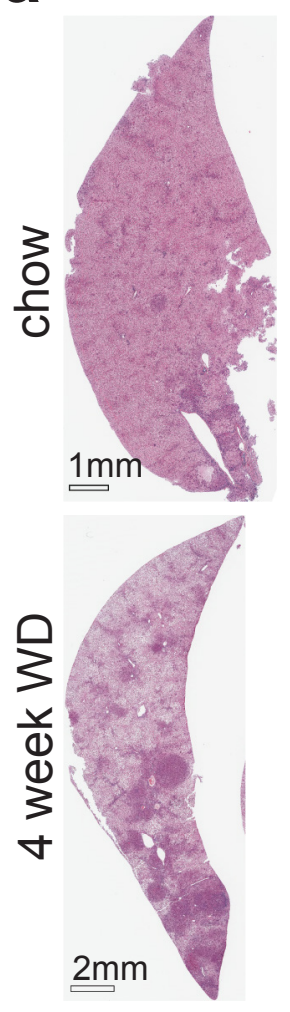

b

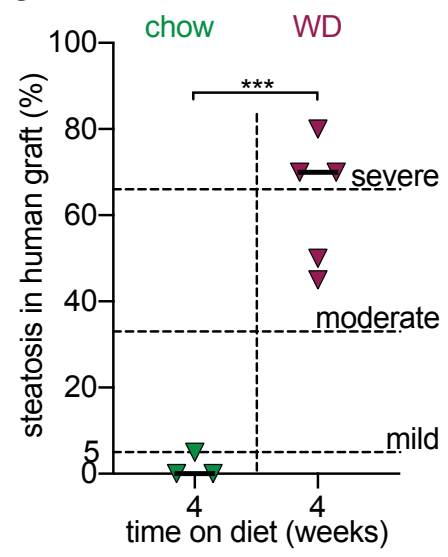

C

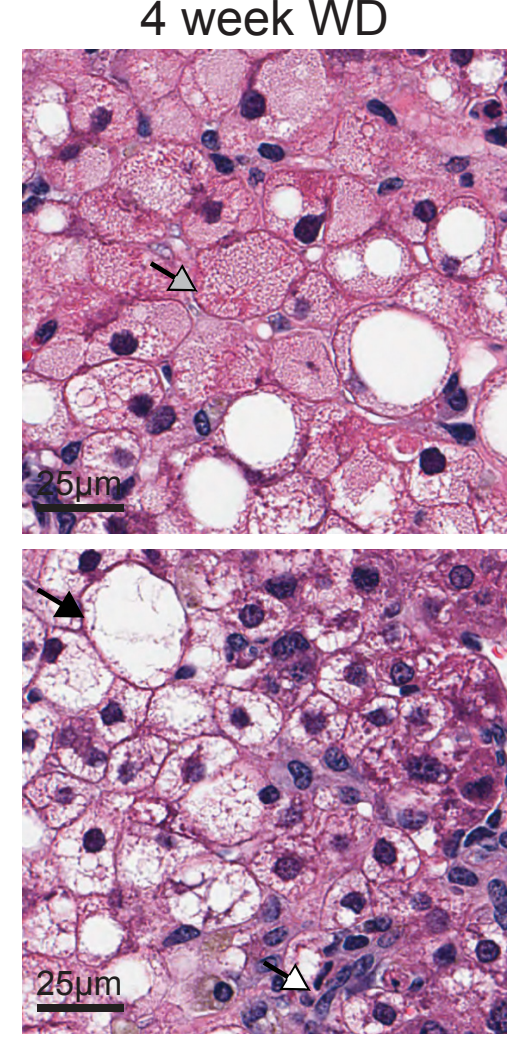

d

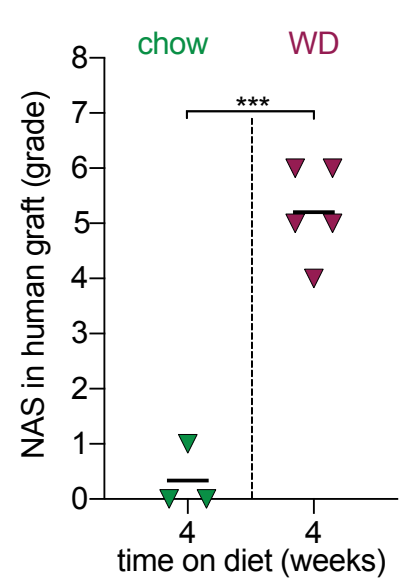

g

$\Delta$ WD 148M-huFRG

vS.

$\Delta$ WD 148I-huFNRG

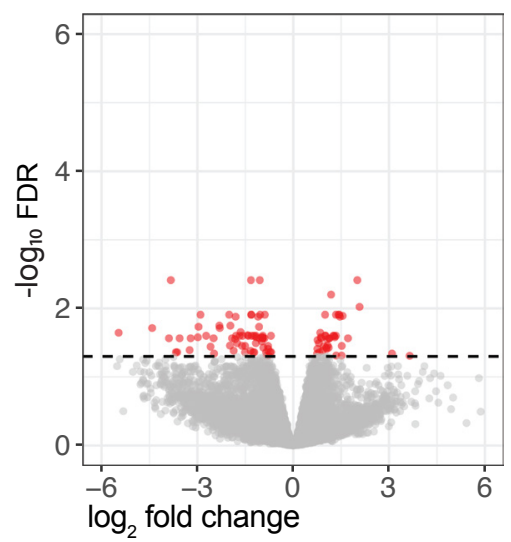

e

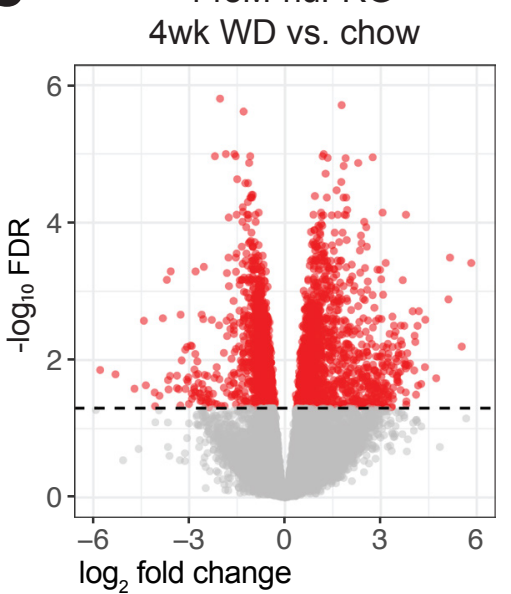

f

small molecule metabolic process oxoacid metabolic process oxidation-reduction process carboxylic acid metabolic process oxidoreductasse activity monocarboxylic acid metabolic process small molecule biosynthetic process lipid metabolic process steroid metabolic process carboxylic acid catabolic process

organic acid catabolic process alpha-amino acid metabolic process sterol metabolic process fatty acid metabolic process cholesterol biosynthetic process cellular amino acid metapolic process regulation of sterol biosynthetic process regulation of cholesterol biosynthetic process organic acid biosynthetic process active transmembrane transporter activity Activation of gene expression by SREBF (SREBP

carboxylic acid biosynthetic process

Extracellular matrix organization lipid catabollic process long-chain fatty acid metabolic process cellular lipid catabolic process cellular metabolic process mitochondrial membrane mitochondrial matrix electron transport chain oxidative phosphorylation oxidative phosphorylation
respiratory electron transport chain ATP synthesis coupled electron transport mitochondrial ATP synthesis coupled electron transport

negative regulation of cellular process regulation of cell communication regulation of signal transduction

regulation of hydrolase activity lipid oxidation proteasome-mediated ubiquitin-dependent protein catabolic process
acetyl-CoA metabolic process fatty-acyl-CoA binding
fatty acid beta-oxidation fatty acid alpha-oxidation - . . $-\log ($ value)

- 0.25

- 0.50

- 2.50

- 5.00

- 10.00

Direction

- 20.00

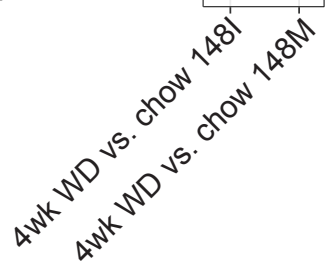


Figure 6: PNPLA3-148M overexpression in hepatocytes exacerbates steatosis in mice on WD

a

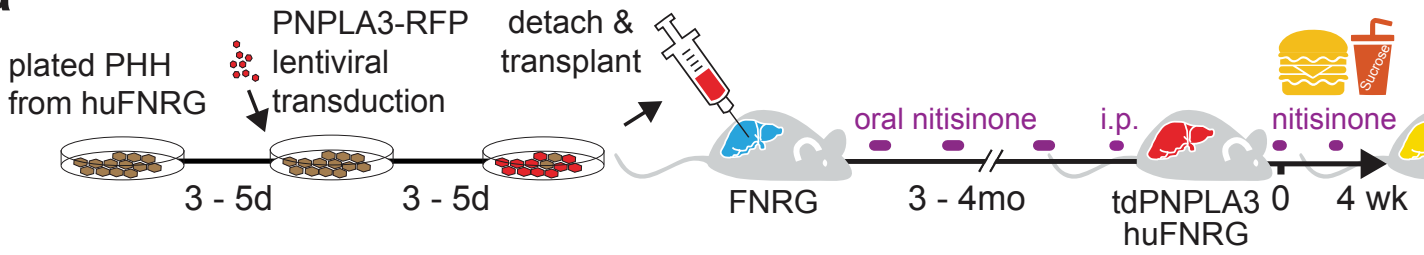

b tdRFP WD
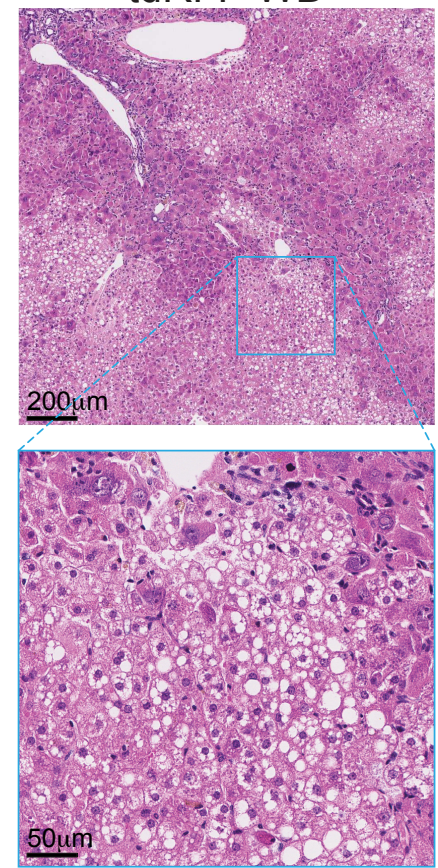

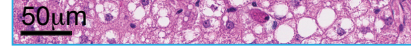
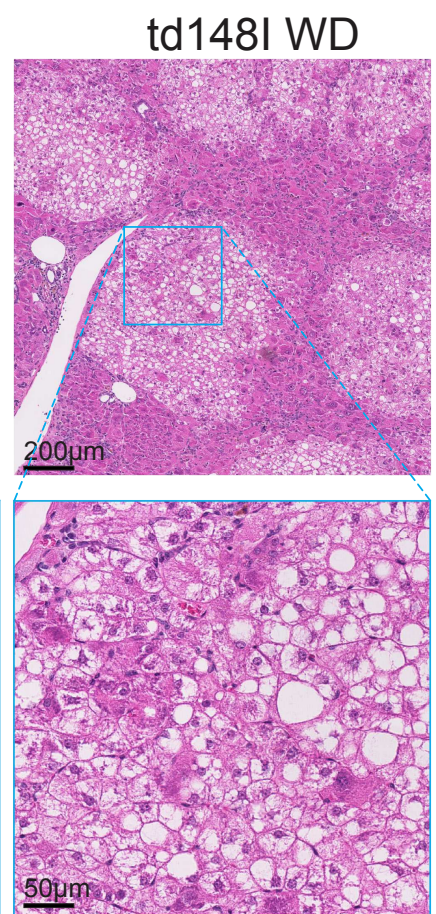
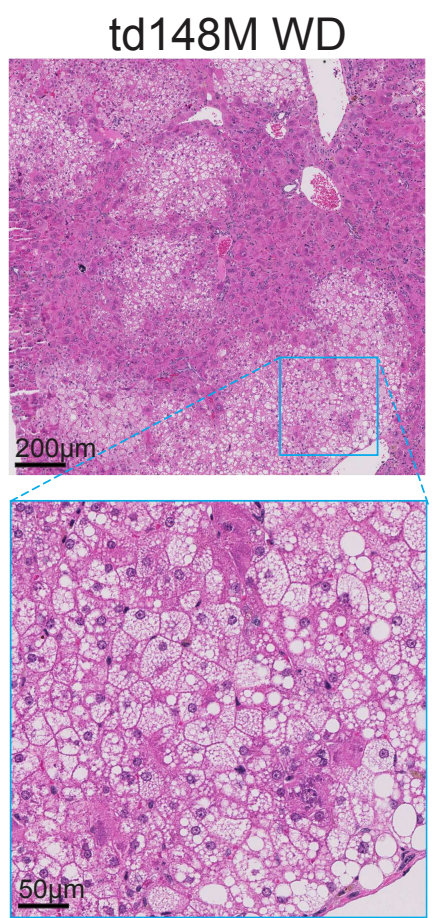

f

e

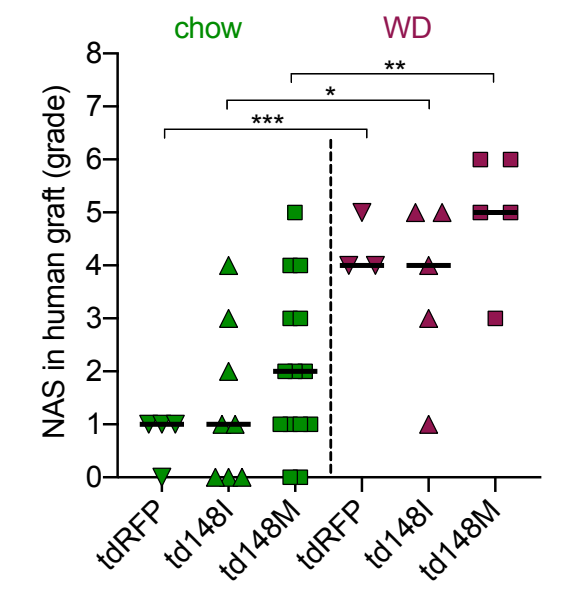

e
td148I vs. td148M - WD

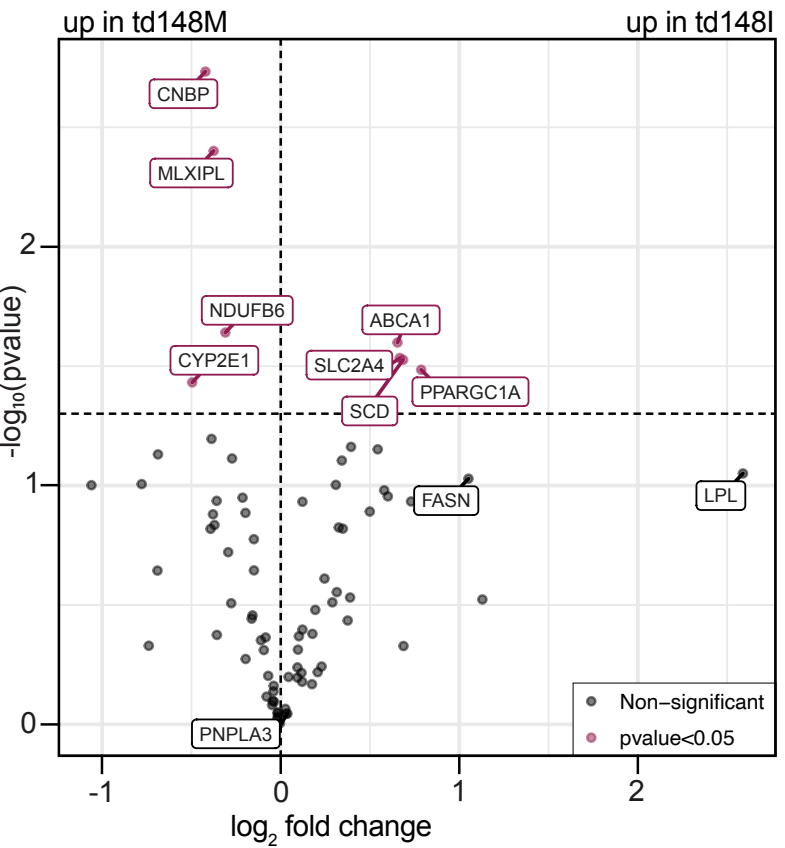

d

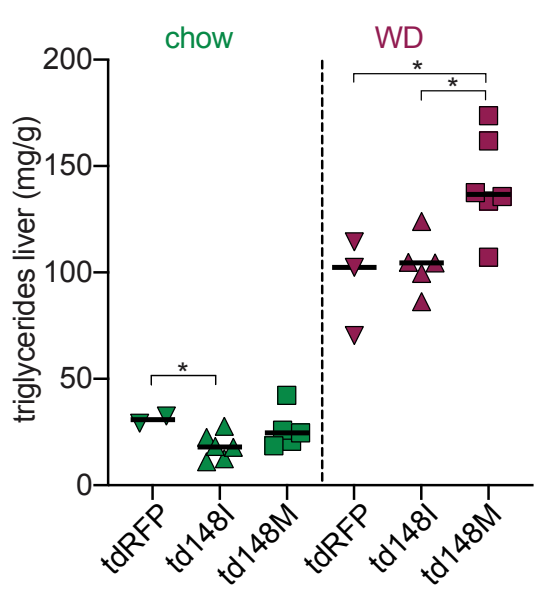

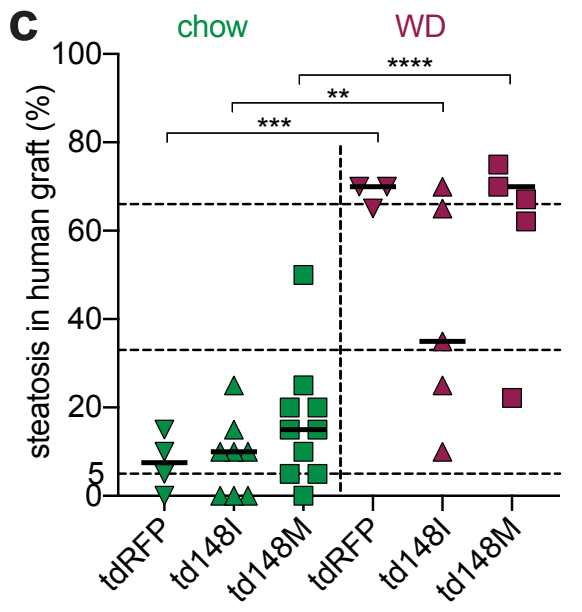


Figure 7: PNPLA3-148M overexpression worsens steatohepatitis on HFD
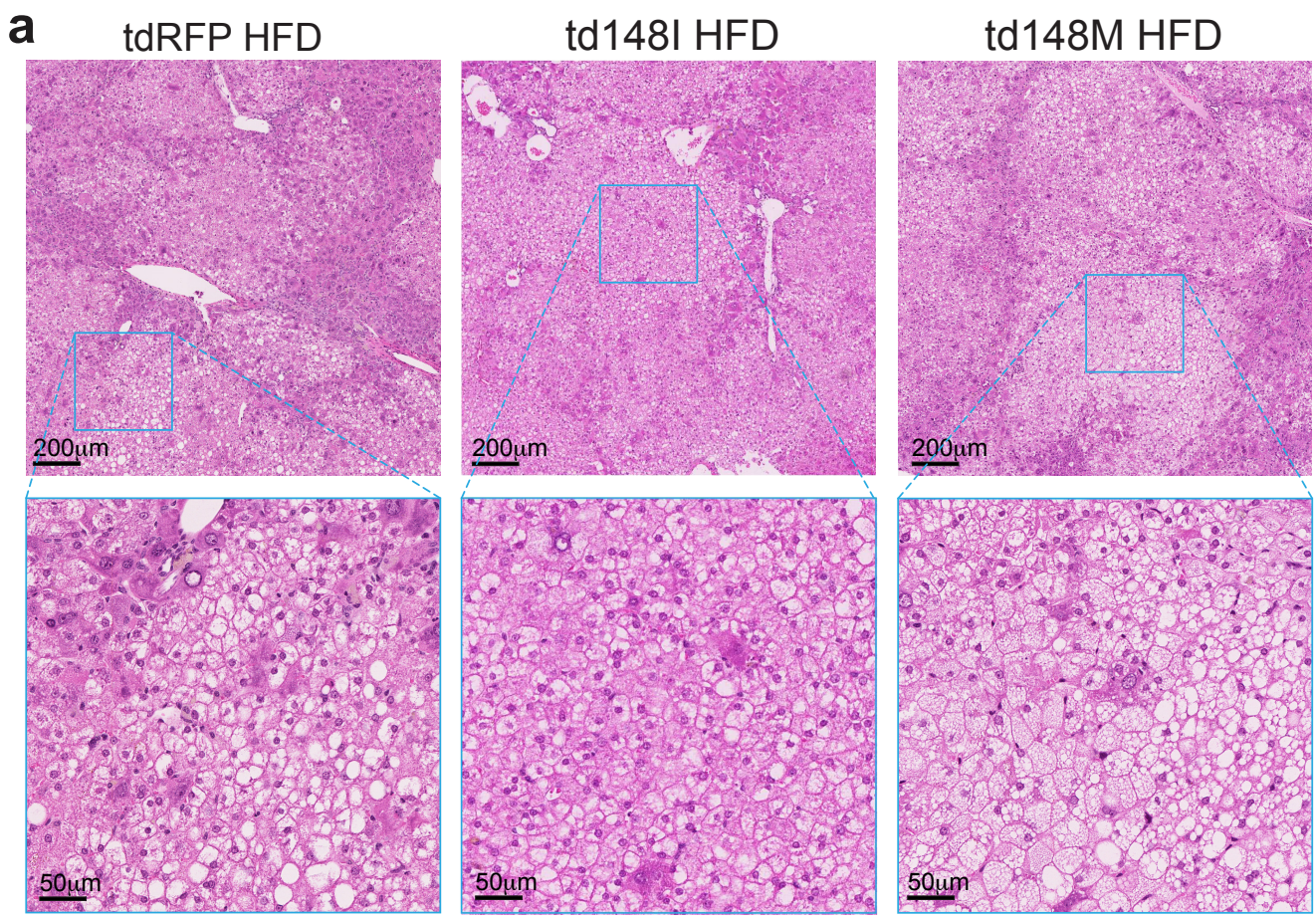

b

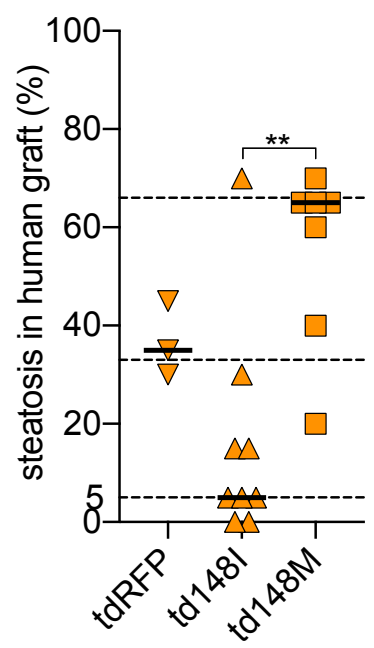

C

td148M HFD

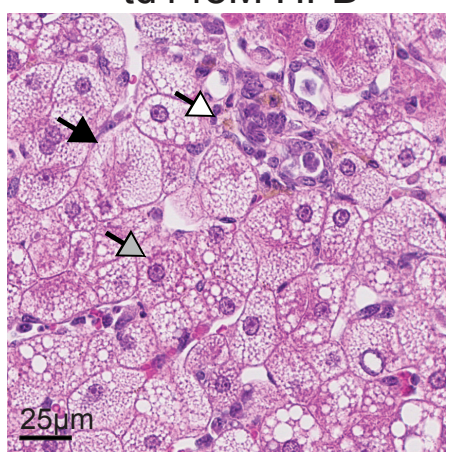

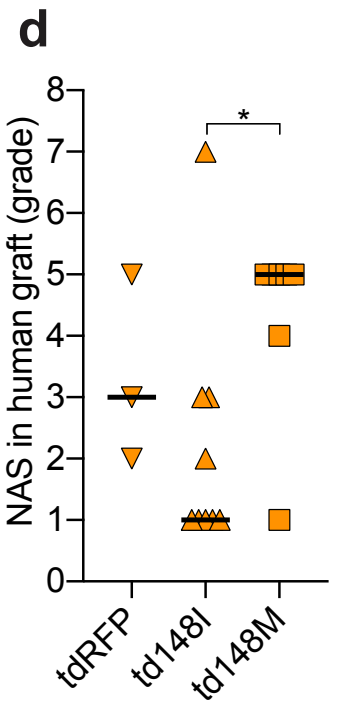

e
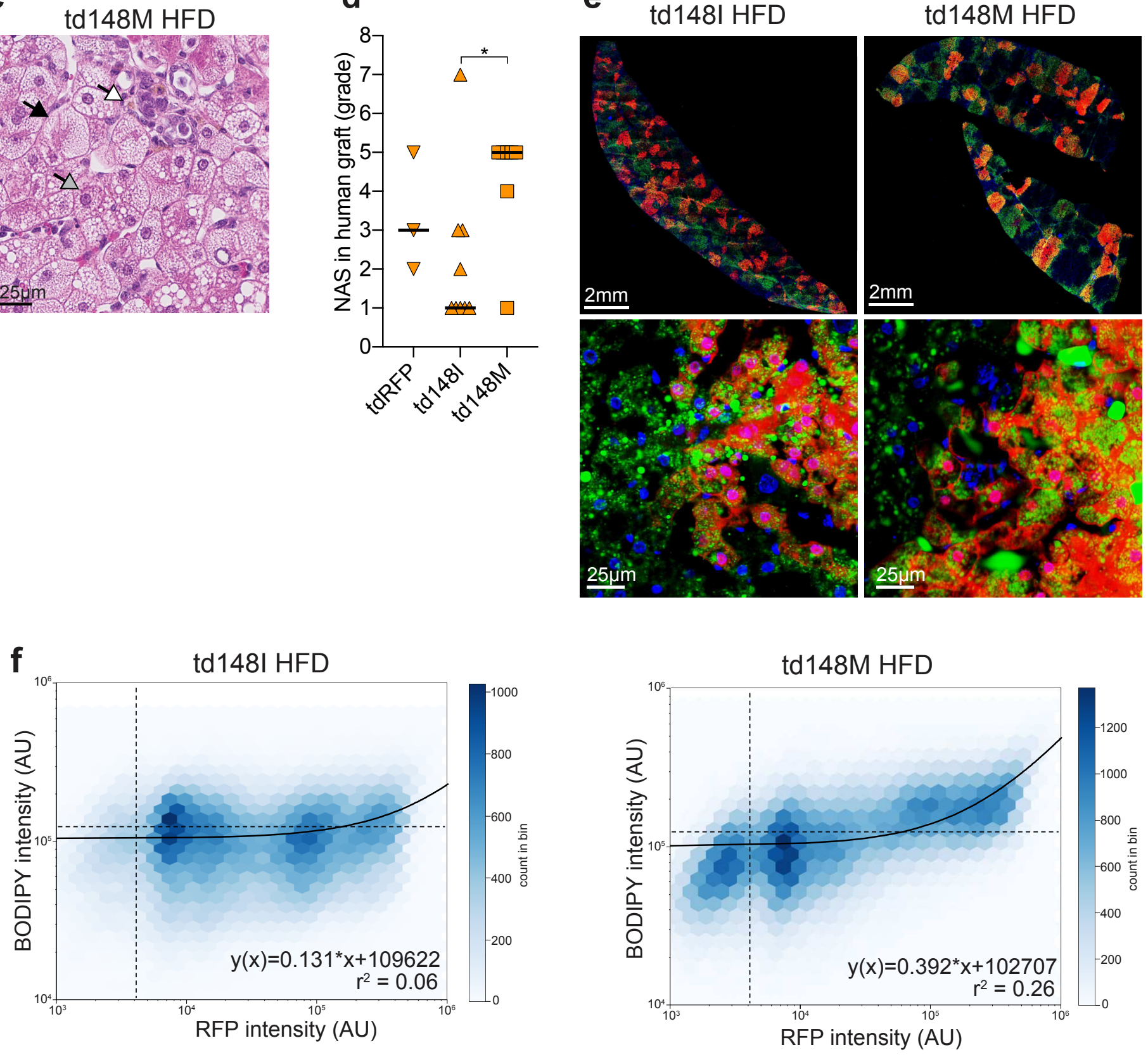
Supplemental Figure S1: Human hepatocyte steatosis in chimeric models
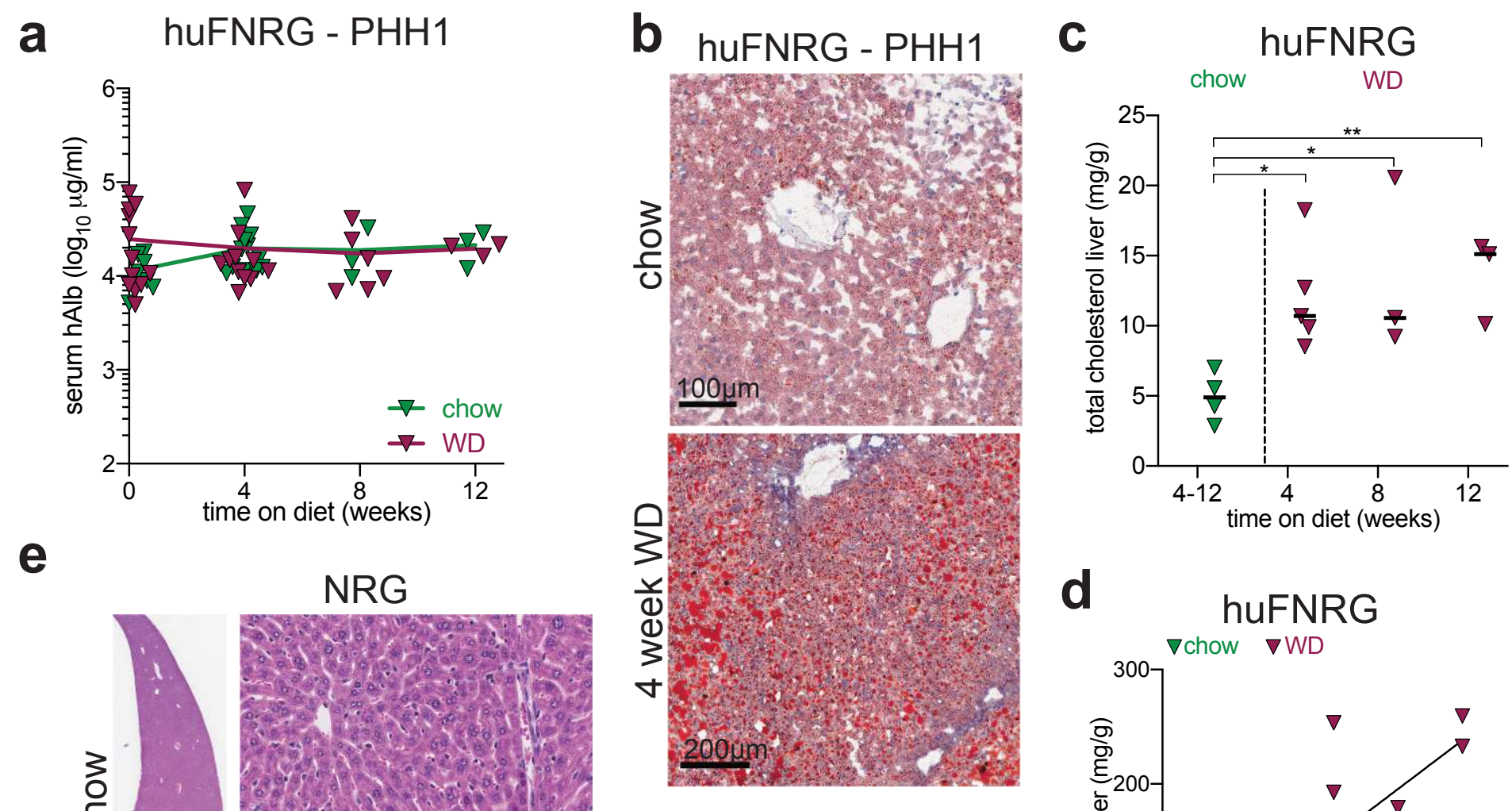

e
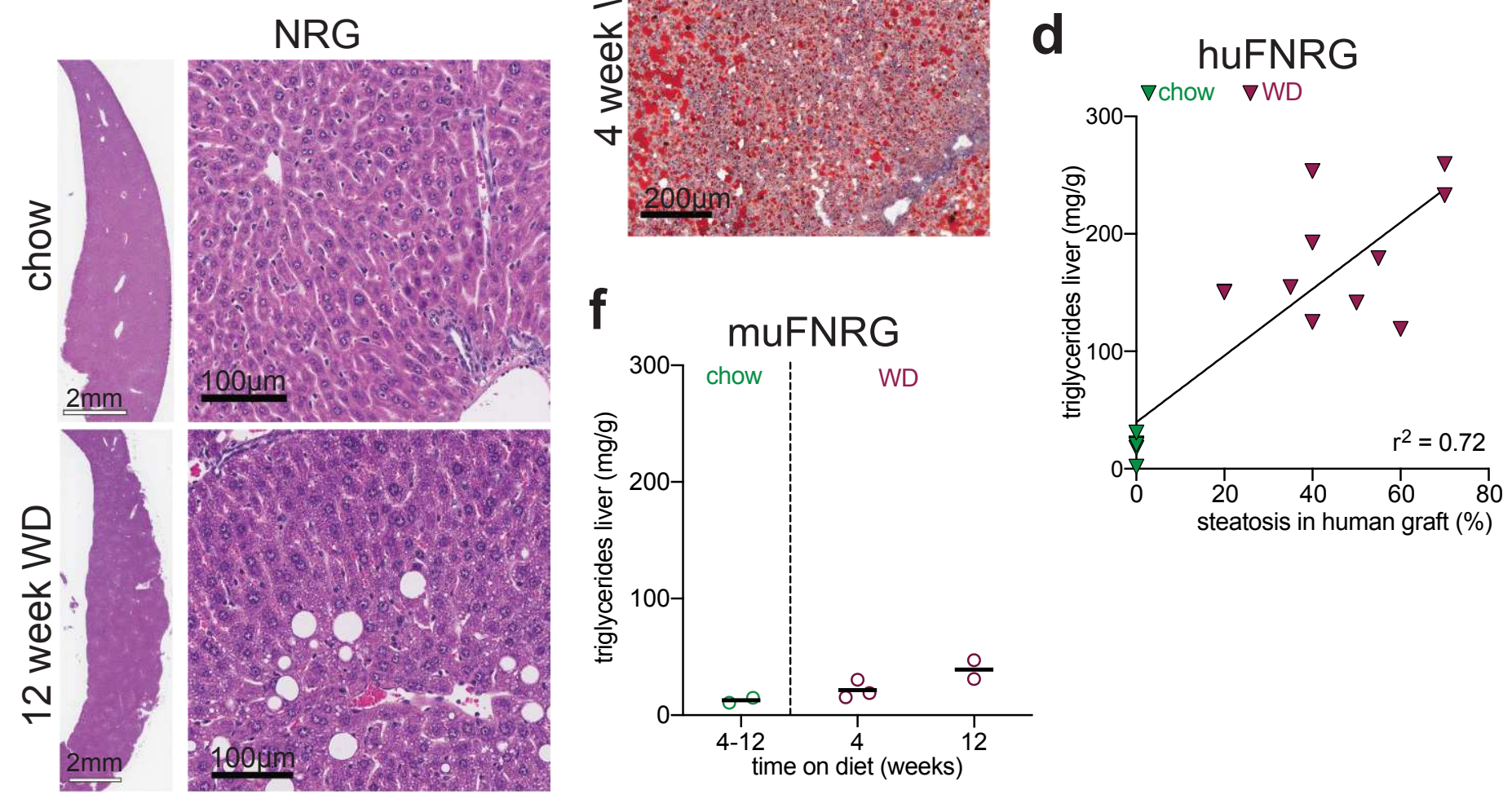

9 huFNRG - PHH2 huTK-NOG - PHH2

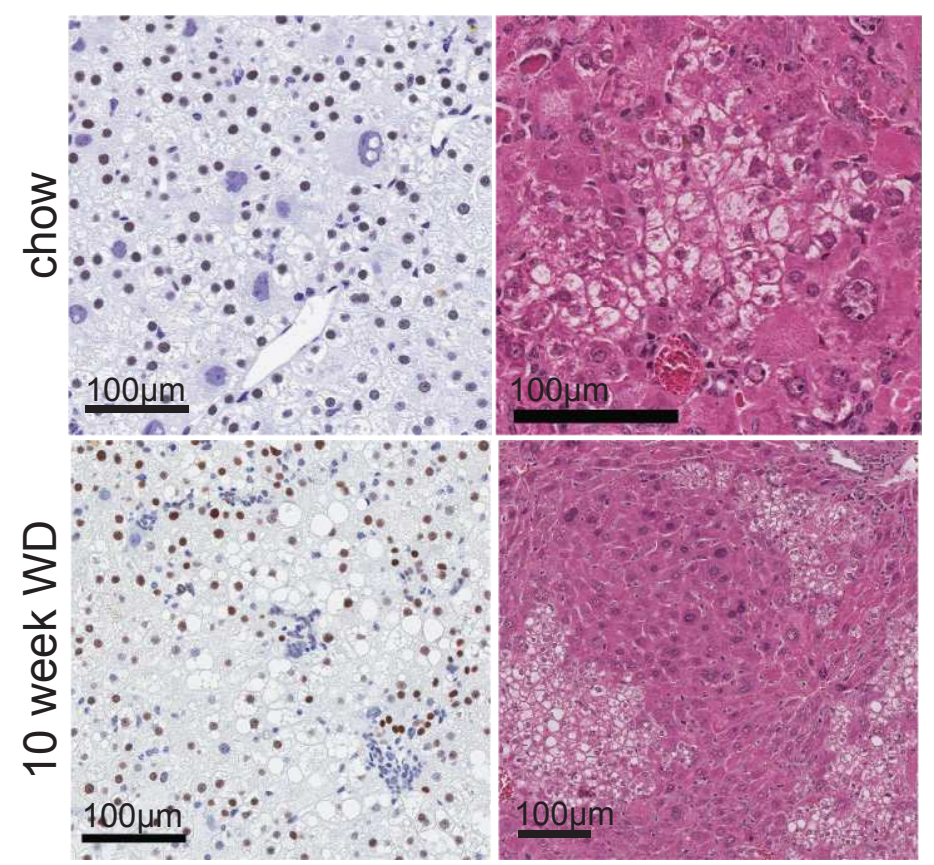

h

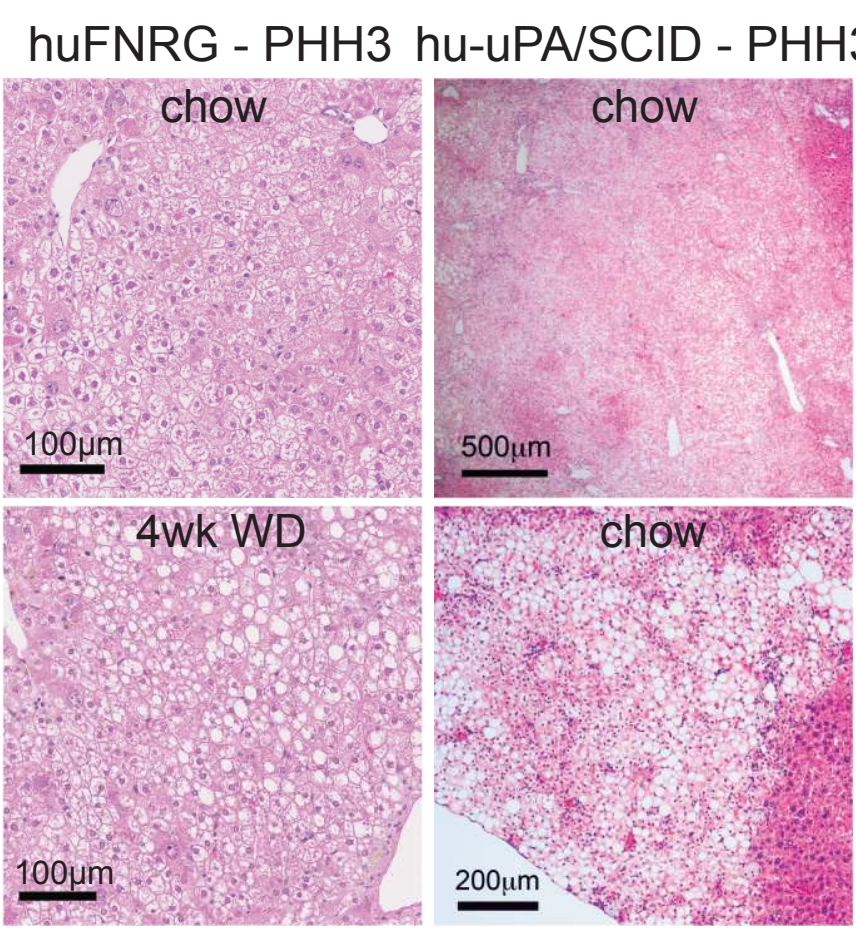


Supplemental Figure S2: Systemic metabolic effects of WD feeding

a

cholesterol profile

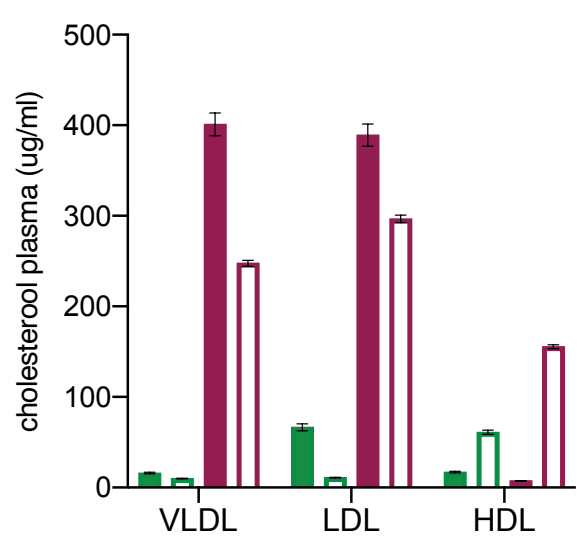

C

gluclose tolerance

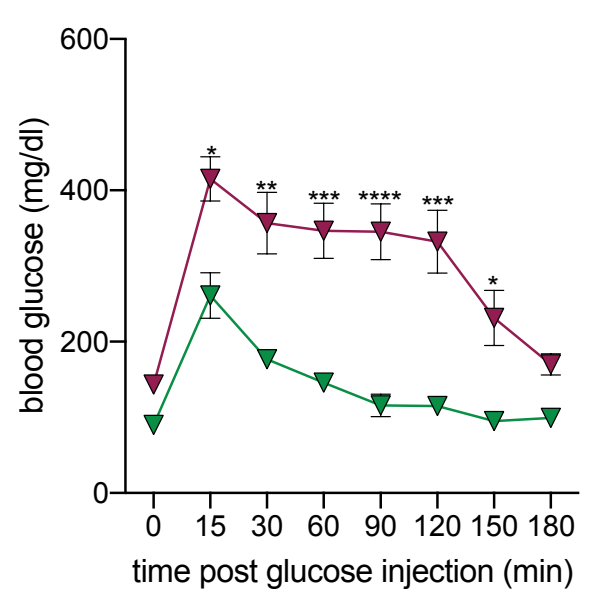

b

triglyceride profile

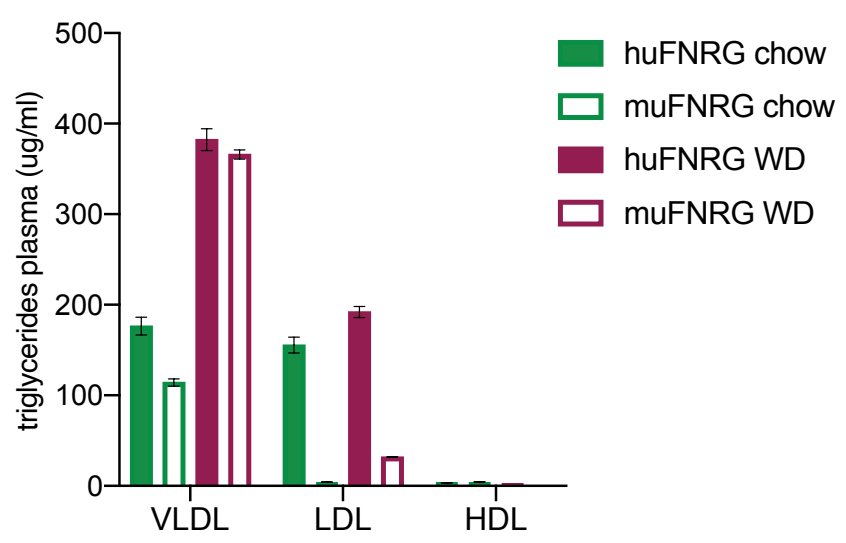

d

$\nabla$ huFNRG WD

$\rightarrow$ huFNRG chow

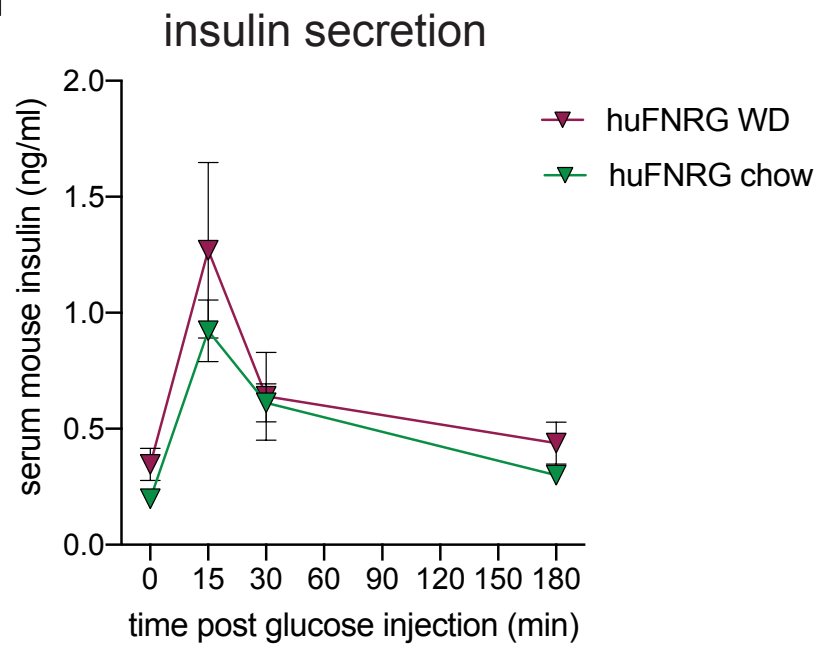


Supplemental Figure S3: Mild steatohepatitis in huFNRG mice on WD

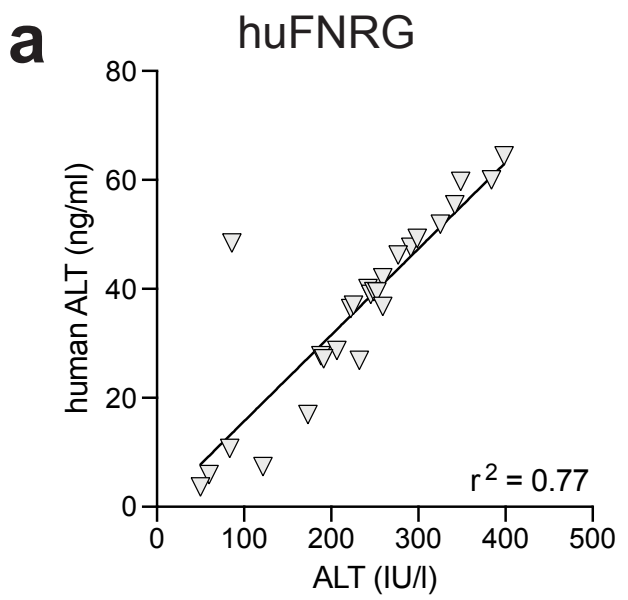

b
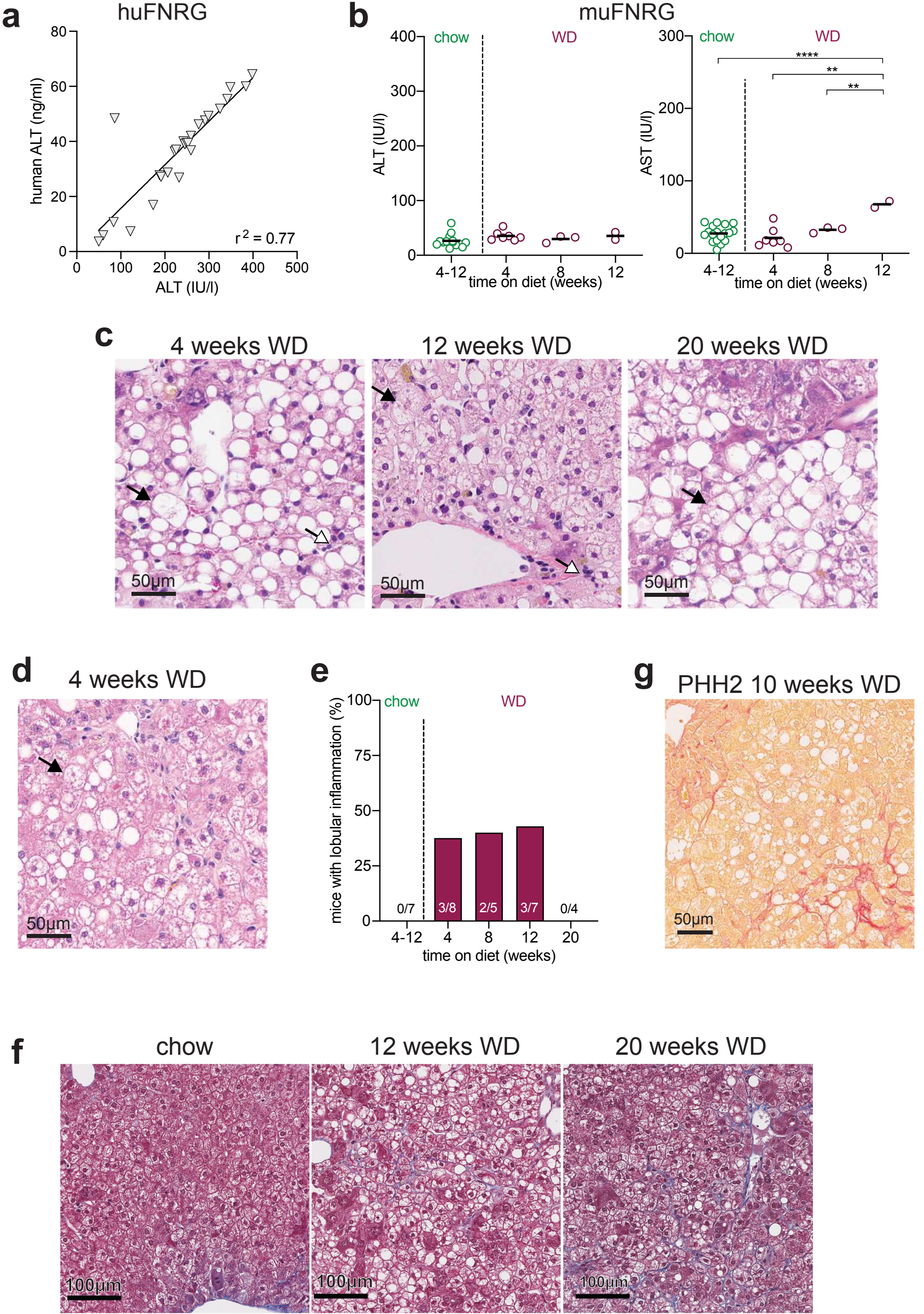
Supplemental Figure S4: Transcriptional changes in huFNRG mice on Western Diet

a

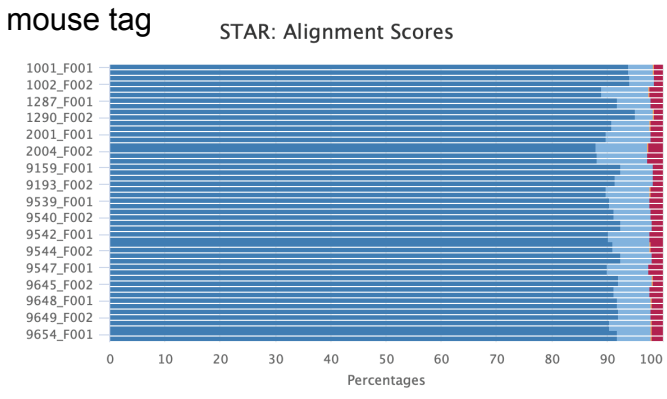

Uniquely mapped Mapped to multiple loci Mapped to too many loc

b

human pathways

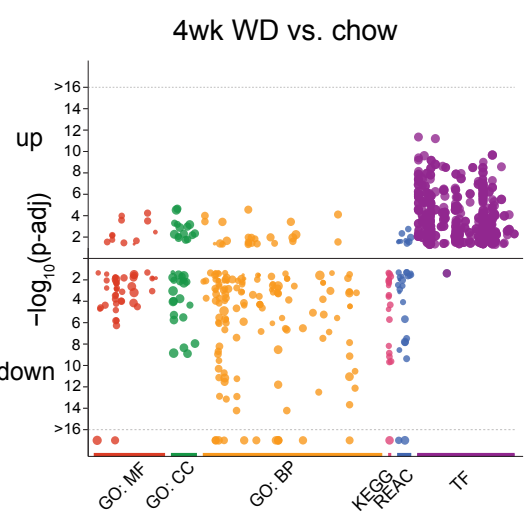

8wk WD vs. 4 wk WD

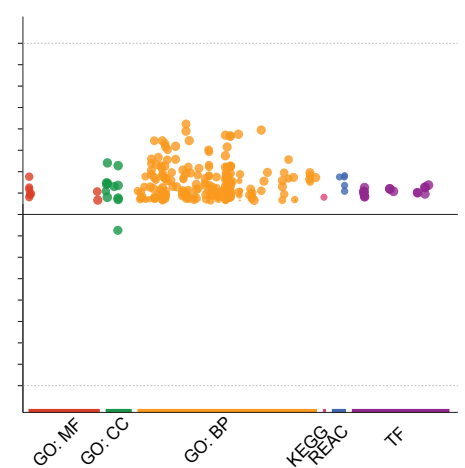

$12 w k$ WD vs. $8 w k$ WD

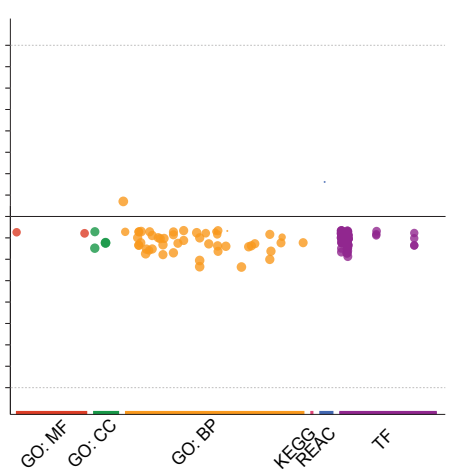

$20 w k$ WD vs. $12 w k$ WD

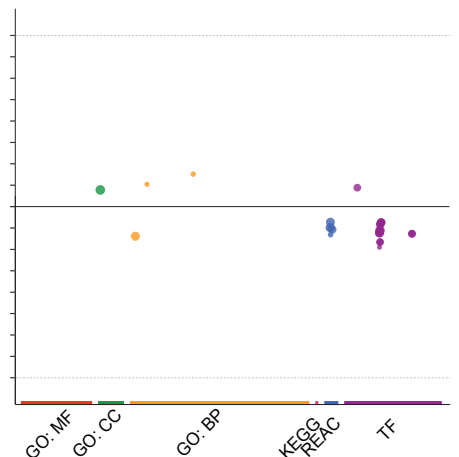

C

mouse pathways
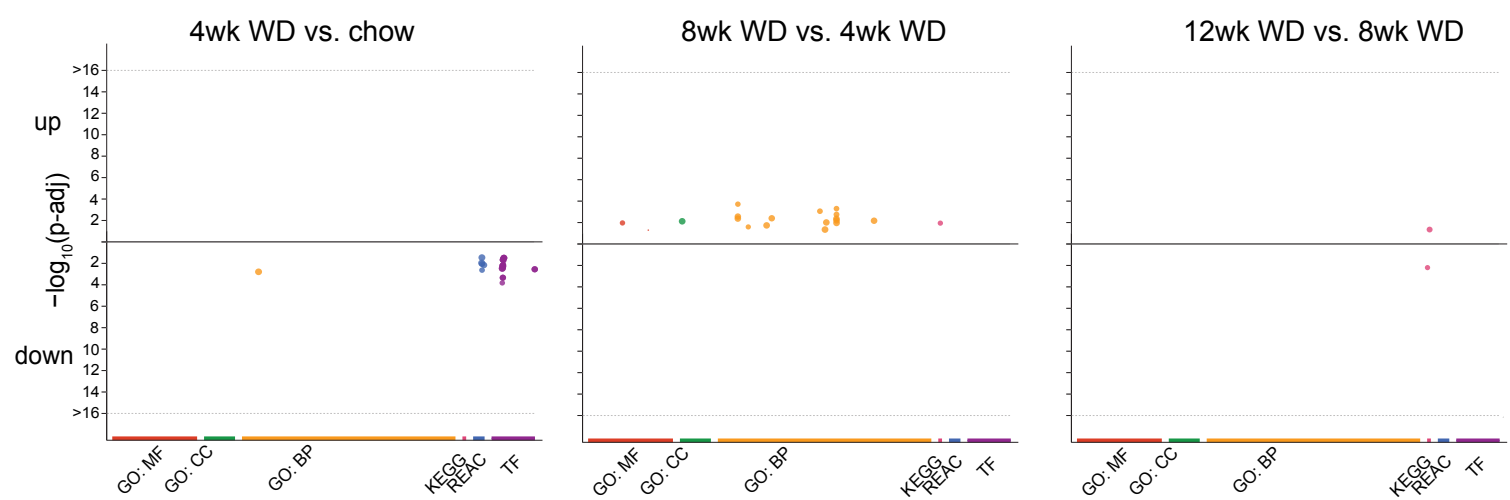

20wk WD vs. 12 wk WD

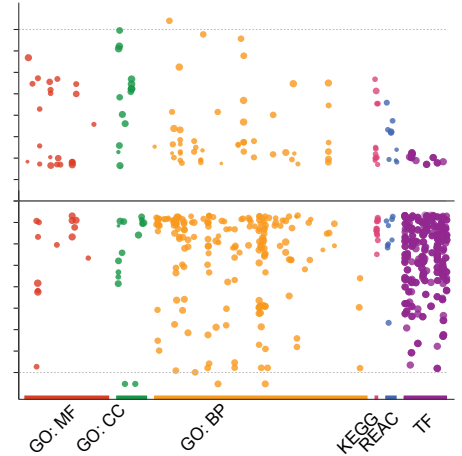


Supplemental Figure S5: 148M-huFRG mice develop steatohepatitis
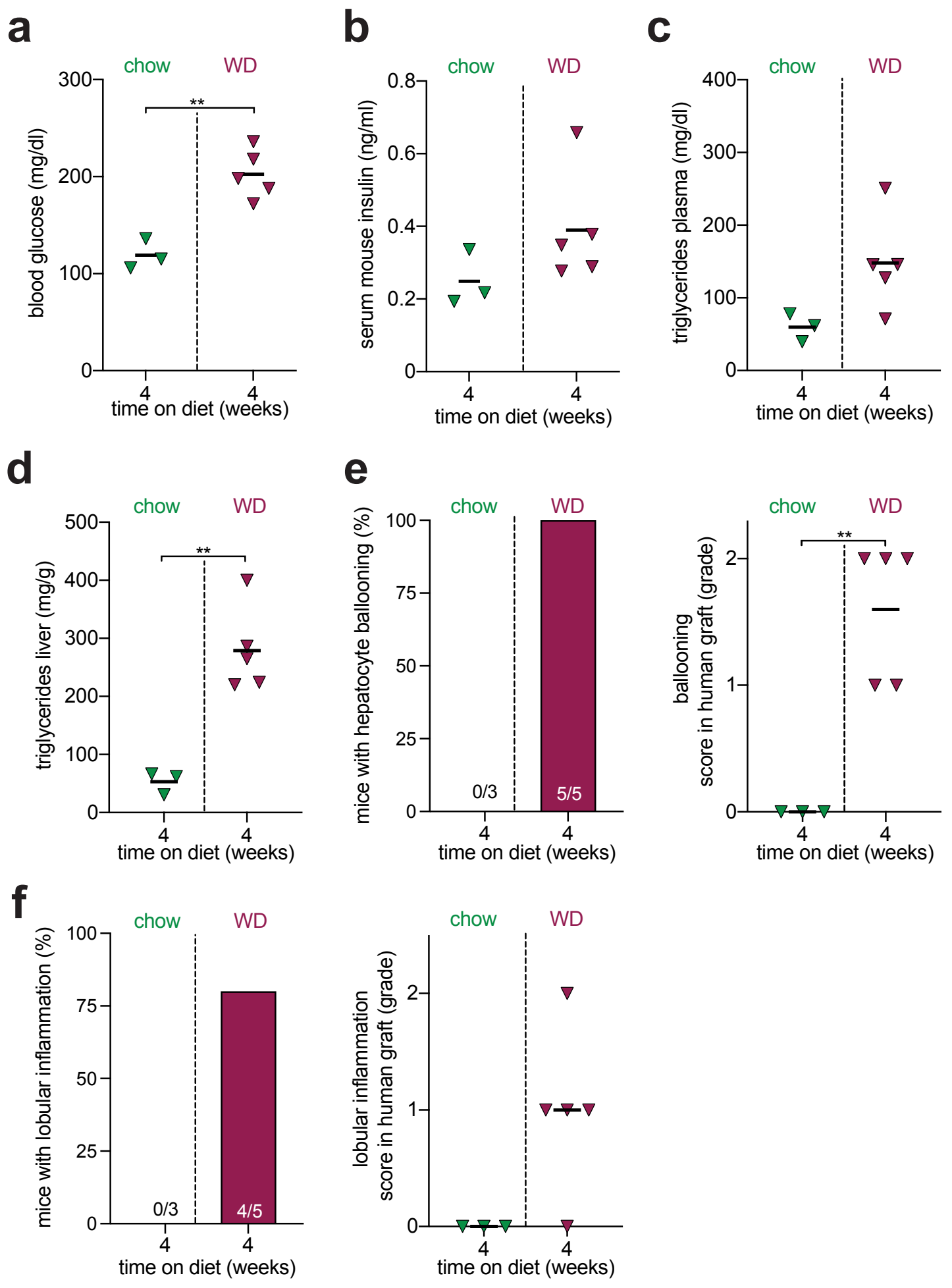

9 chow 148M-huFRG
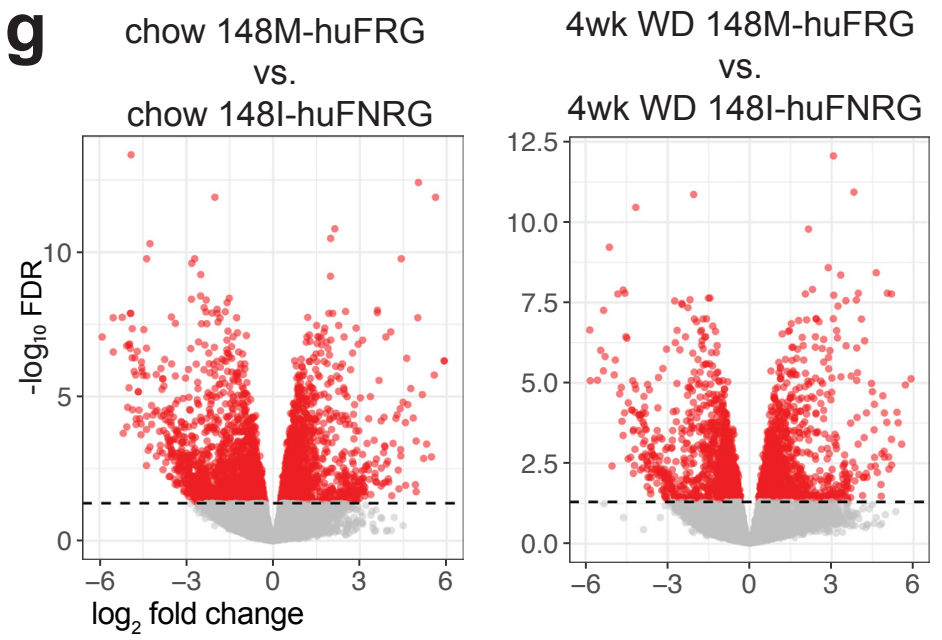

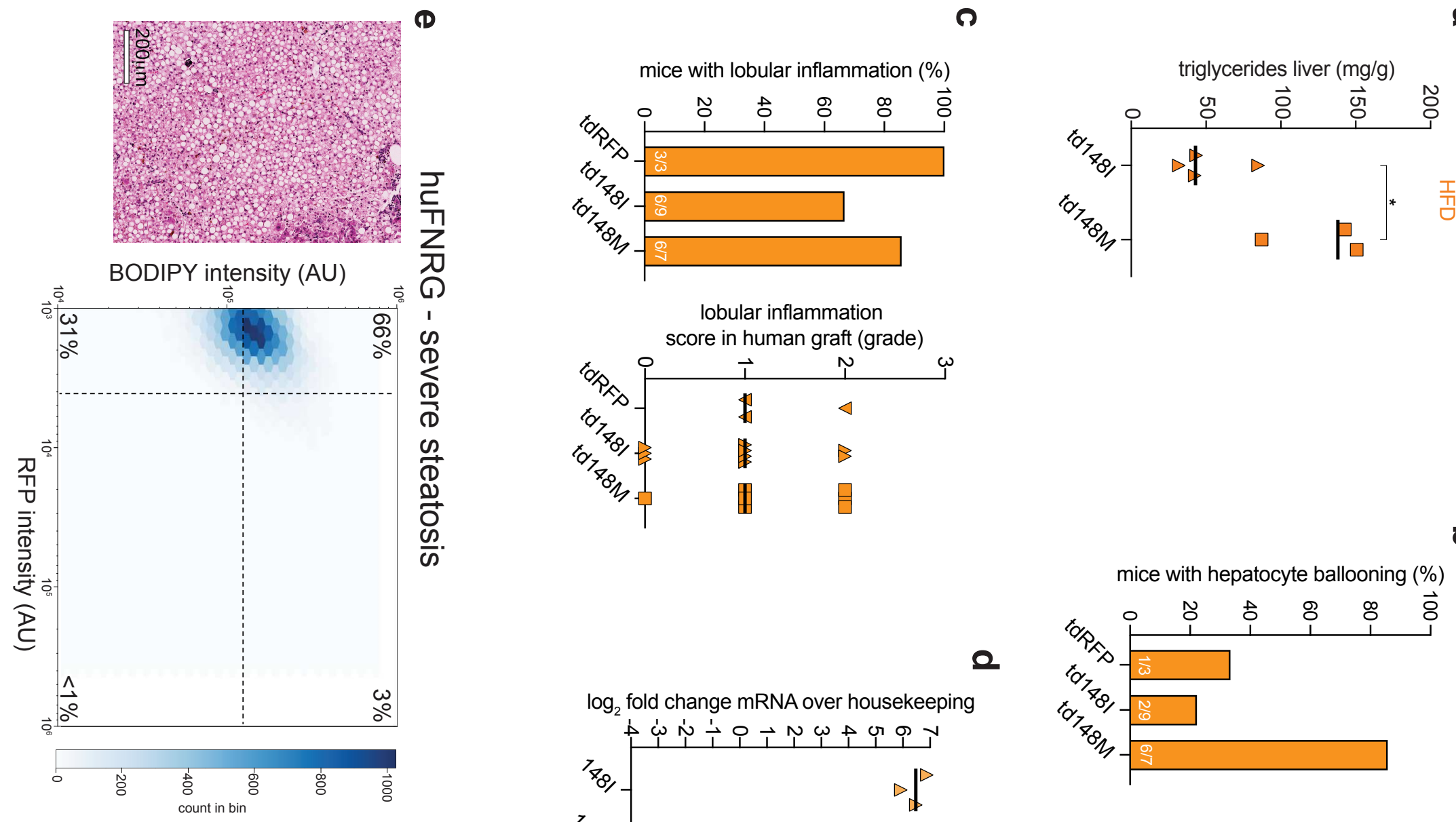

lobular inflammation

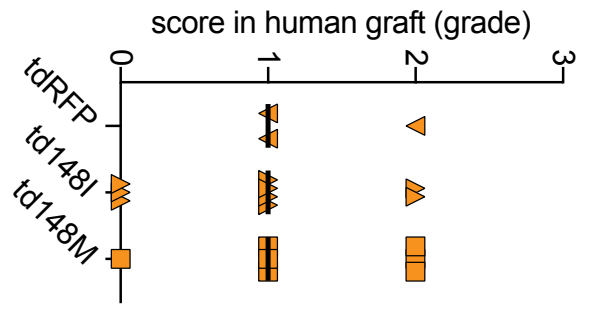

.

$\log _{2}$ fold change mRNA over housekeeping
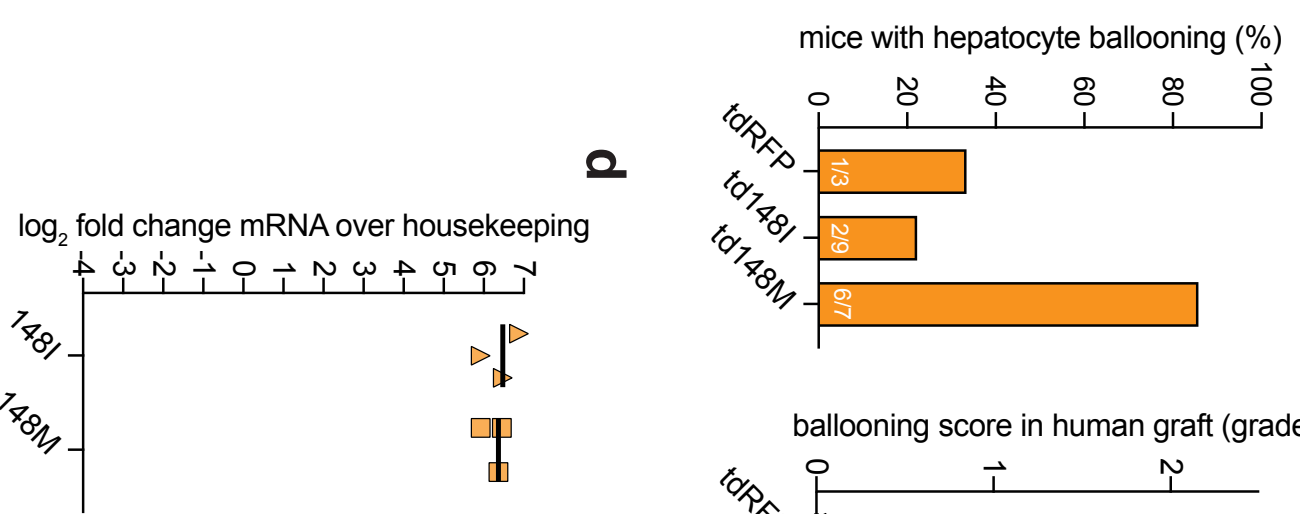

ballooning score in human graft (grade)

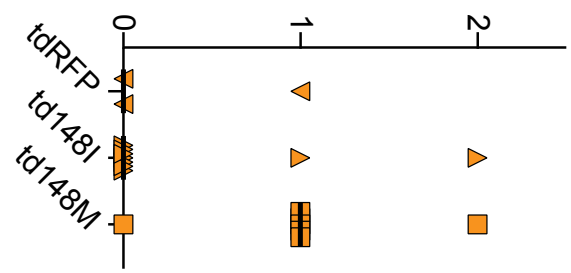

$\sigma$ 


\begin{tabular}{|c|c|c|c|c|c|c|}
\hline \multirow[b]{2}{*}{ Gene } & \multicolumn{3}{|c|}{ Average fold change over housekeeping - chow } & \multicolumn{3}{|c|}{ Average fold change over housekeeping - WD } \\
\hline & tdRFP & $\operatorname{td} 148$ & $\operatorname{td} 148 \mathrm{M}$ & tdRFP & $\operatorname{td} 148$ & $\operatorname{td} 148 \mathrm{M}$ \\
\hline ABCA1 & 0.368883198 & 0.267786579 & 0.5519846 & 0.6727912 & 0.707126819 & 0.449371439 \\
\hline ACACA & 0.035656185 & 0.038552958 & 0.071810784 & 0.083214261 & 0.083744866 & 0.057474499 \\
\hline ACADL & 0.110949301 & 0.101655054 & 0.14558227 & 0.199057575 & 0.170211114 & 0.188835791 \\
\hline ACLY & 0.436496378 & 0.567439241 & 0.52725121 & 0.461086724 & 0.497637348 & 0.653387539 \\
\hline ACOX1 & 2.517925637 & 2.849879635 & 2.231809941 & 4.178923865 & 3.903006829 & 3.150915163 \\
\hline ACSL5 & 1.798792185 & 1.662574057 & 1.742976738 & 2.444098549 & 1.72579322 & 1.822920344 \\
\hline ACSM3 & 3.176615241 & 4.975173094 & 3.533113634 & 4.922244267 & 3.695856521 & 5.951371558 \\
\hline ADIPOR1 & 0.433077354 & 0.428142874 & 0.482731662 & 0.513066692 & 0.503029756 & 0.537061101 \\
\hline ADIPOR2 & 0.833701393 & 0.851964307 & 0.911126783 & 1.078684175 & 1.05454579 & 0.831764838 \\
\hline AKT1 & 0.238494562 & 0.272889999 & 0.25423422 & 0.325361344 & 0.323277443 & 0.272738552 \\
\hline APOA1 & 101.5835357 & 91.40737221 & 104.0803431 & 181.4814453 & 183.2887107 & 205.1508232 \\
\hline APOB & 34.41468389 & 34.00765282 & 40.64313743 & 54.92296325 & 49.88464552 & 53.8572082 \\
\hline APOC3 & 90.65886756 & 75.41545032 & 105.3560986 & 128.6632013 & 128.3388654 & 132.7015488 \\
\hline APOE & 14.36420394 & 12.37371322 & 15.63639277 & 22.92161029 & 21.05691049 & 19.42476157 \\
\hline ATP5C1 & 1.883365035 & 2.025512372 & 1.77809252 & 2.120542084 & 1.699034415 & 1.970308135 \\
\hline CASP3 & 0.14222822 & 0.138794303 & 0.153920158 & 0.178177748 & 0.169408443 & 0.173943887 \\
\hline CD36 & 1.109397909 & 0.964592474 & 1.11226837 & 2.520850775 & 2.150983894 & 2.280733948 \\
\hline CEBPB;CEBPB-AS1 & 0.131348551 & 0.150504893 & 0.230620196 & 0.345507675 & 0.234725837 & 0.180984119 \\
\hline CNBP & 1.681373003 & 1.631270687 & 1.4649865 & 1.764718361 & 1.404109004 & 1.880379885 \\
\hline CPT1A & 1.573508999 & 1.988468625 & 1.018277411 & 1.336678777 & 1.157082822 & 0.946755159 \\
\hline CPT2 & 0.860227974 & 0.828165 & 0.66481186 & 1.060712454 & 0.996925551 & 1.027784841 \\
\hline CYP2E1 & 48.13382884 & 52.98359038 & 50.65613294 & 98.46466494 & 71.22366345 & 100.4290926 \\
\hline CYP7A1 & 0.045502776 & 0.056265985 & 0.845162194 & 1.302704706 & 0.418776654 & 0.873183109 \\
\hline DGAT2 & 1.785244728 & 1.27057123 & 2.648681604 & 4.05056992 & 2.756586266 & 3.585261025 \\
\hline FABP1 & 53.76484075 & 60.30053405 & 32.83167347 & 73.11974507 & 69.42785371 & 65.05980676 \\
\hline FABP3 & 0.030896467 & 0.060774155 & 0.014648243 & 0.01640808 & 0.019624525 & 0.012184946 \\
\hline FABP5 & 0.051886321 & 0.044810817 & 0.03213479 & 0.032659698 & 0.026736462 & 0.030655666 \\
\hline FAS & 0.111562643 & 0.086802296 & 0.13182671 & 0.154132372 & 0.163275093 & 0.152697716 \\
\hline FASN & 0.559186083 & 0.570116112 & 1.225495927 & 1.890858451 & 2.355251867 & 1.136443648 \\
\hline FOXA2 & 0.103035877 & 0.10728501 & 0.142773019 & 0.177348528 & 0.158534305 & 0.160535032 \\
\hline FOXO1 & 0.120158742 & 0.148166229 & 0.083402316 & 0.089057933 & 0.067803239 & 0.068487858 \\
\hline G6PC & 12.92404672 & 12.05760327 & 9.598385617 & 6.984889807 & 6.97351766 & 9.122014223 \\
\hline G6PD & 0.009800051 & 0.014160332 & 0.010899059 & 0.01559439 & 0.013913829 & 0.016862318 \\
\hline GCK & $8.3343 \mathrm{E}-05$ & $9.51975 \mathrm{E}-05$ & 0.003181612 & 0.001282137 & 0.003028274 & 0.001825874 \\
\hline GK & 0.143295743 & 0.171232355 & 0.144058926 & 0.212581321 & 0.183416553 & 0.210181879 \\
\hline GSK3B & 0.207578414 & 0.276604229 & 0.228618434 & 0.281228441 & 0.257897685 & 0.241680432 \\
\hline HMGCR & 0.15452151 & 0.165345921 & 0.212269244 & 0.13726636 & 0.147313581 & 0.112117061 \\
\hline HNF4A & 3.996306081 & 3.943692904 & 4.753606452 & 5.331060791 & 4.639432231 & 5.688434883 \\
\hline IGF1 & 0.010426567 & 0.004866301 & 0.020359608 & 0.00306642 & 0.003730813 & 0.002496448 \\
\hline IGFBP1 & 12.44027242 & 13.34406143 & 10.08440111 & 9.935696835 & 7.082208029 & 9.074199535 \\
\hline IL1B & $8.3343 \mathrm{E}-05$ & $9.51975 \mathrm{E}-05$ & 0.000153504 & 0.000171541 & 0.000355026 & 0.00059247 \\
\hline INSR & 0.183237252 & 0.195447769 & 0.175270148 & 0.222821746 & 0.180882517 & 0.177165927 \\
\hline IRS1 & 0.205456659 & 0.246516791 & 0.237258747 & 0.24300858 & 0.238575745 & 0.240730114 \\
\hline LDLR & 0.074089788 & 0.071929665 & 0.150844332 & 0.076538055 & 0.08279066 & 0.054625664 \\
\hline LEPR & 0.391030638 & 0.420141832 & 0.210327956 & 0.118092904 & 0.137903343 & 0.11766551 \\
\hline LPL & 0.000305595 & 0.003088181 & $8.52238 \mathrm{E}-05$ & 0.000824859 & 0.001619555 & 0.000269268 \\
\hline MAPK1 & 0.216294145 & 0.24540175 & 0.202622028 & 0.29092258 & 0.2612777 & 0.240152682 \\
\hline MAPK8 & 0.440281802 & 0.442547892 & 0.437249105 & 0.673947769 & 0.541084062 & 0.548329977 \\
\hline MLXIPL & 0.981203864 & 1.023470324 & 1.673085147 & 1.834284935 & 1.644262293 & 2.133155067 \\
\hline MTOR & 0.17906682 & 0.182718973 & 0.172394208 & 0.29297052 & 0.213826751 & 0.196764821 \\
\hline NDUFB6 & 2.083029561 & 1.521543606 & 1.555686354 & 1.911428134 & 1.58798678 & 1.968871463 \\
\hline NFKB1 & 0.106767086 & 0.102503414 & 0.111532414 & 0.152805067 & 0.151704325 & 0.147149618 \\
\hline NR1H2 & 0.114479501 & 0.110169063 & 0.104124579 & 0.137119984 & 0.130467613 & 0.134134529 \\
\hline NR1H3 & 0.247463587 & 0.287596294 & 0.268097208 & 0.400125993 & 0.338054687 & 0.433478265 \\
\hline NR1H4 & 0.201521892 & 0.207478544 & 0.292981797 & 0.440805966 & 0.34670219 & 0.418768654 \\
\hline PCK2 & 4.062199455 & 3.364462956 & 4.718561546 & 4.495995215 & 4.841278589 & 4.233615961 \\
\hline PDK4 & 1.112647549 & 1.749907845 & 0.555580537 & 1.678436521 & 1.128671641 & 0.977784582 \\
\hline PIK3CA & 0.054329087 & 0.057465596 & 0.053603142 & 0.070732218 & 0.061532335 & 0.068284189 \\
\hline PIK3R1 & 0.448423342 & 0.564011593 & 0.681658373 & 0.734037504 & 0.701808132 & 0.564470116 \\
\hline PKLR & 0.449193735 & 0.517960062 & 0.604507878 & 1.085207053 & 1.076839318 & 1.392419297 \\
\hline PNPLA3 & 0.113741573 & 42.36533239 & 19.63691814 & 0.41869652 & 25.73235028 & 25.07571438 \\
\hline PPA1 & 3.355371555 & 3.922263647 & 2.695813337 & 2.900275121 & 2.583081448 & 4.431660341 \\
\hline PPARA & 0.721905106 & 0.909120989 & 0.938163465 & 1.131783972 & 0.943743907 & 0.946628258 \\
\hline PPARG & 0.050849692 & 0.052560543 & 0.053859743 & 0.118730057 & 0.111007887 & 0.103434765 \\
\hline PPARGC1A & 1.281363665 & 1.791011309 & 1.195756063 & 1.032135858 & 1.224376566 & 0.709771484 \\
\hline PRKAA1 & 0.123766016 & 0.136691229 & 0.134870277 & 0.121180919 & 0.132680989 & 0.136364862 \\
\hline PTPN1 & 0.058334826 & 0.045048939 & 0.056797798 & 0.072605707 & 0.063509352 & 0.070857878 \\
\hline RBP4 & 47.4800469 & 47.0333149 & 53.55747394 & 55.1223592 & 56.63062069 & 59.44587287 \\
\hline RXRA & 0.47999656 & 0.456585111 & 0.594743968 & 1.003442925 & 0.886003198 & 0.783381395 \\
\hline$S C D$ & 1.508170175 & 1.266519099 & 3.007627677 & 8.603733964 & 9.775838241 & 6.078768955 \\
\hline SERPINE1 & 0.096467782 & 0.08181576 & 0.179991825 & 0.694545795 & 0.710336881 & 0.324698447 \\
\hline SLC27A5 & 3.237334403 & 2.818938018 & 4.087306836 & 4.617318743 & 4.981965166 & 3.976890967 \\
\hline SLC2A1 & 0.011493495 & 0.010800383 & 0.020086921 & 0.00544164 & 0.00680493 & 0.006026506 \\
\hline SLC2A2 & 2.953556671 & 2.595344705 & 4.167931432 & 7.107000571 & 6.062238245 & 5.953809267 \\
\hline SLC2A4 & 0.005322738 & 0.004416619 & 0.005518313 & 0.009949256 & 0.010087355 & 0.006353036 \\
\hline SOCS3 & $8.3343 \mathrm{E}-05$ & $9.51975 \mathrm{E}-05$ & 0.000832534 & 0.00011565 & $8.5228 \mathrm{E}-05$ & 0.000137509 \\
\hline SREBF1 & 0.624108037 & 0.65410058 & 0.72348495 & 1.386376188 & 1.341956211 & 0.949654207 \\
\hline SREBF2 & 0.089736092 & 0.070610763 & 0.145507148 & 0.1244603 & 0.132038588 & 0.103745968 \\
\hline STAT3 & 0.700090113 & 0.810089398 & 0.848016211 & 0.849374371 & 0.849885479 & 0.649254289 \\
\hline XBP1 & 2.455458823 & 2.230970953 & 3.150858997 & 3.678411889 & 3.944201872 & 3.625131871 \\
\hline
\end{tabular}


Figures
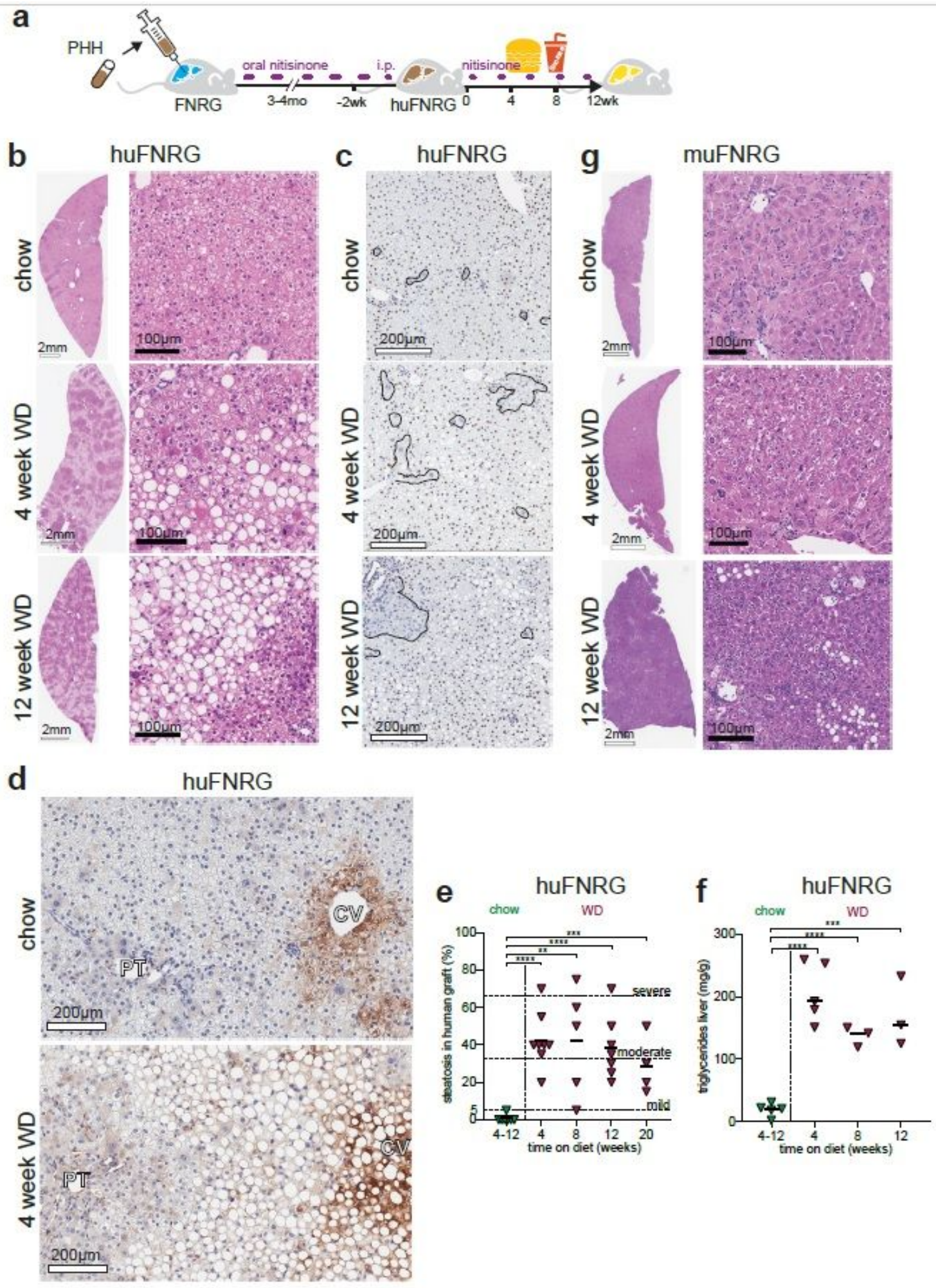

Figure 1

Human hepatocytes in chimeric mice on WD rapidly develop steatosis a) Experimental timeline of FNRG mice that were transplanted with primary human hepatocytes $(\mathrm{PHH})$, cycled off nitisinone to create huFNRG mice, and then subjected to chow or WD for up to 12 weeks. b) H\&E staining on livers from 
huFNRG mice on chow and after 4 or 12 weeks on WD. c) Staining against human nuclear mitotic apparatus- 1 in livers of huFNRG mice on chow and 4 or 12 weeks on WD. Areas with predominantly mouse hepatocytes are outlined. d) Staining against human glutamine synthetase on livers from huFNRG mice on chow and 4 weeks on WD. Central vein (CV), portal tract (PT). e) Liver steatosis score of the human graft in huFNRG mice on chow and after 4 to 20 weeks on WD. Symbols individual mice, bars are median, unpaired t-test with $* * p<0.005, * \star \star p<0.0005$ and $* \star \star \star p<0.0001$. f) Hepatic triglyceride levels in livers of huFNRG mice on chow and after 4 to 12 weeks on WD. Symbols individual mice, bars are median, unpaired t-test with ${ }^{* \star *} p<0.0005$ and ${ }^{* \star * *} \mathrm{p}<0.0001$. g) H\&E staining on livers from muFNRG mice on chow and after 4 or 12 weeks on WD. 
a

huFNRG weight

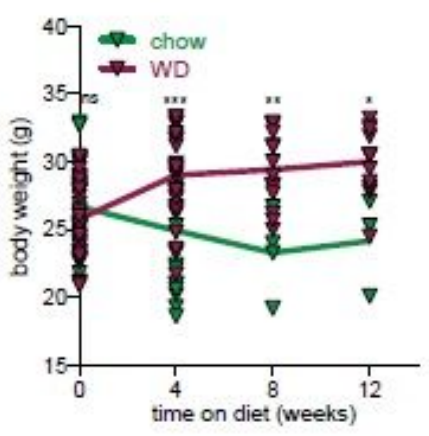

C

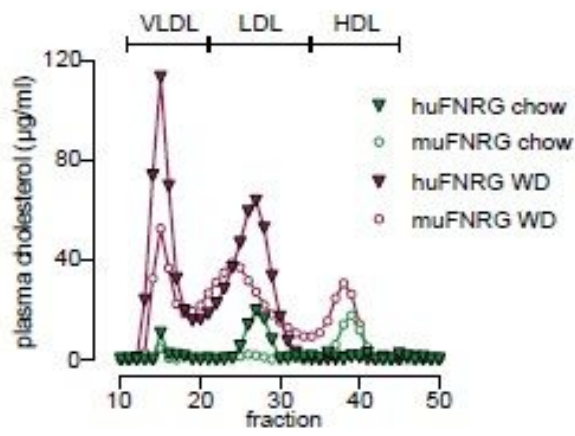

e

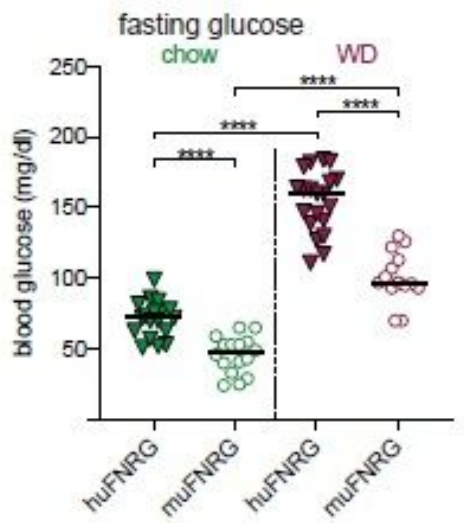

b

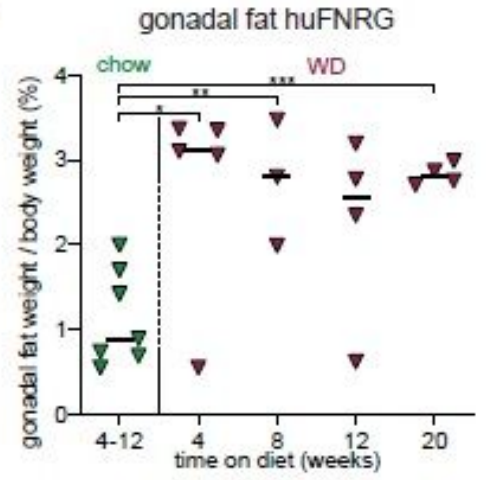

d

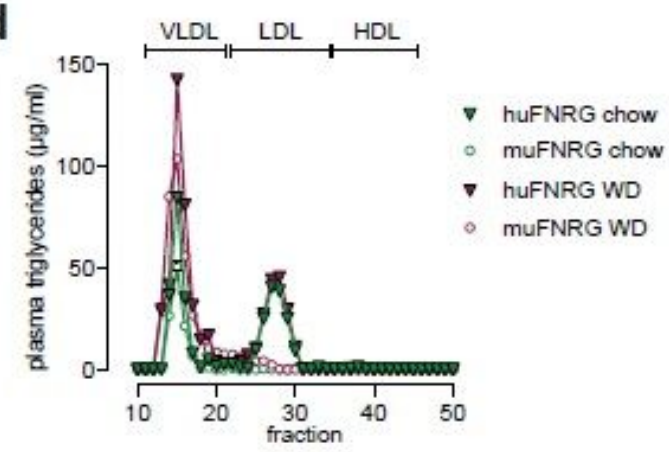

f

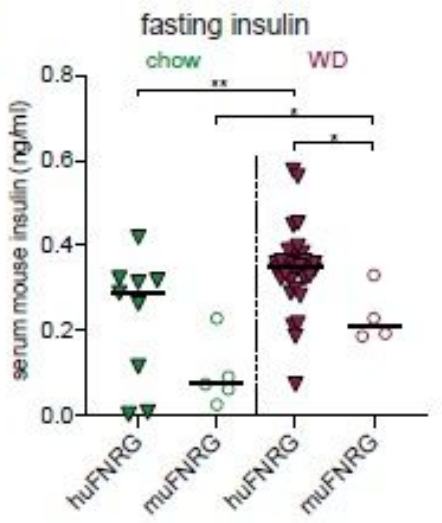

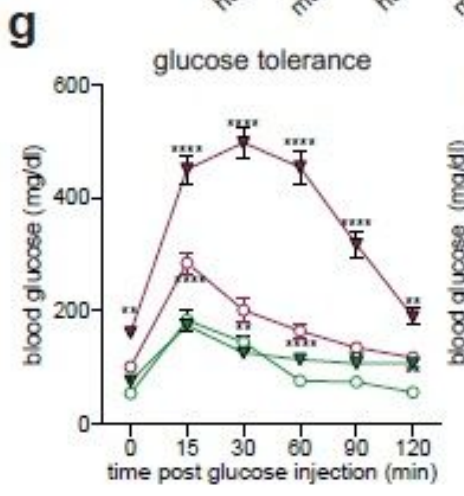

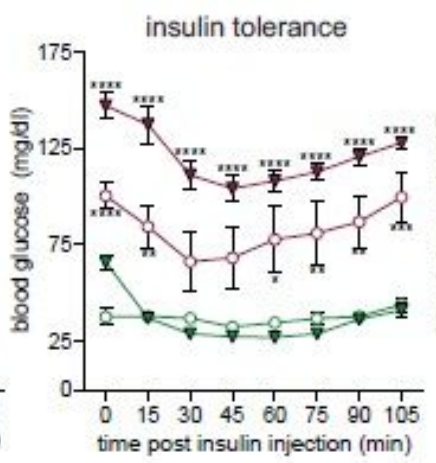

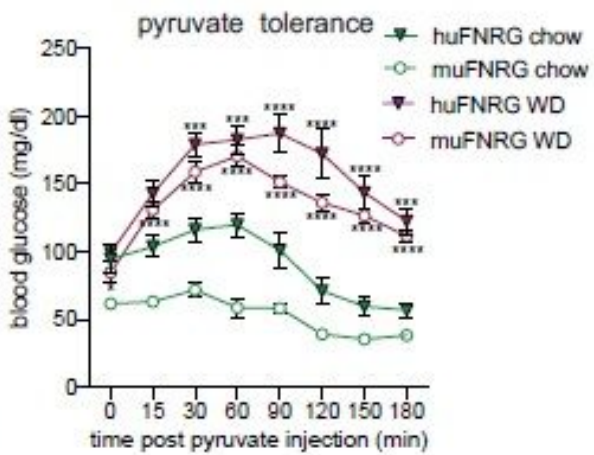

Figure 2

Systemic metabolic effects of WD in chimeric FNRG mice a) Body weight in huFNRG mice during time course of chow or WD feeding. Symbols individual mice, line is mean, two-way ANOVA Sidak pairwise comparison ${ }^{\star} p<0.05,{ }^{* \star} p<0.005,{ }^{* \star *} p<0.0005$. b) Gonadal fat fraction of total body weight in huFNRG mice on chow and after 4 to 20 weeks on WD. Symbols individual mice, bars are median, unpaired t-test with ${ }^{\star} p<0.05,{ }^{* \star} p<0.005,{ }^{* \star} p<0.0005$. c) Cholesterol in plasma lipoprotein fractions from huFNRG and 
muFNRG mice on chow and after 4 weeks on WD. Pooled plasma (3 mice/group), symbols are technical means. d) Triglycerides in plasma lipoprotein fractions from huFNRG and muFNRG mice on chow and after 4 weeks on WD. Pooled plasma (3 mice/group), symbols are technical means. e) Fasting blood glucose from huFNRG and muFNRG mice on chow and after 4 weeks on WD. Symbols individual mice, lines are median, unpaired t-test $* \star \star \star p<0.0001 . f)$ Fasting serum mouse insulin from huFNRG and muFNRG mice on chow 709 and after 4 weeks on WD. Symbols individual mice, lines are median, unpaired t-test with ${ }^{*} p<0.05,{ }^{*} p<0.005 . \mathrm{g}$ ) Intraperitoneal glucose, insulin and pyruvate tolerance testing in huFNRG and muFNRG mice on chow and after 4 weeks on WD. Blood glucose values measured at baseline (0) and at indicated timepoints after i.p. injections with each agent. Symbols are mean SEM of 5-7 mice per group, two-way ANOVA Sidak pairwise comparison of huFNRG on chow and WD ( $\mathrm{p}$ values on top) or muFNRG on chow and WD ( $p$-values in bottom), ${ }^{\star} p<0.05,{ }^{\star *} p<0.005,{ }^{\star \star *} p<0.0005$ and ${ }^{* \star * \star} p<0.0001$ 
a

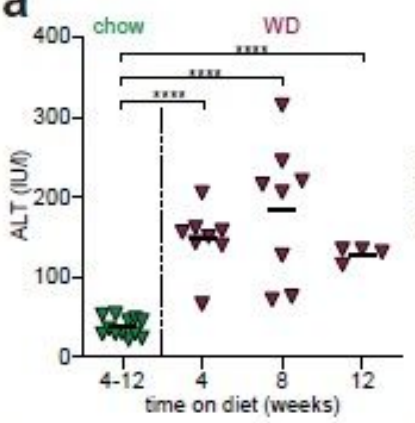

C huFNRG - 4wk WD
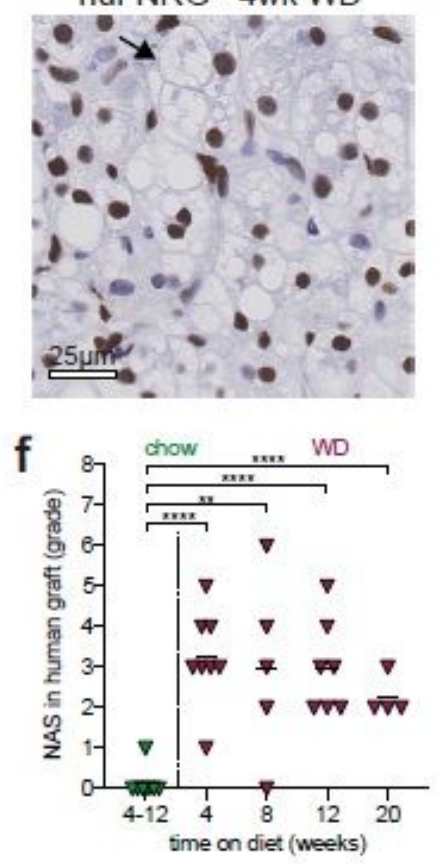

b

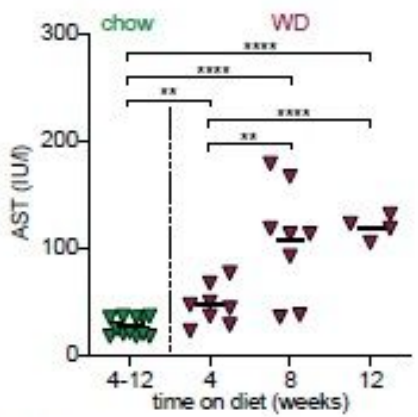

d

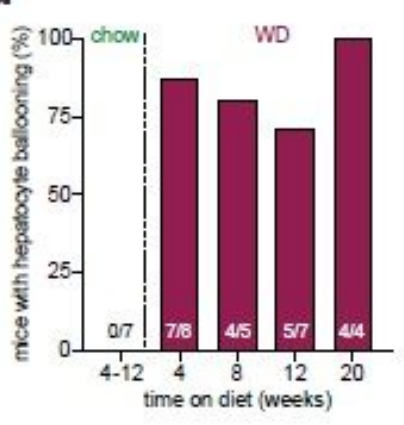

e
huFNRG - 8wk WD
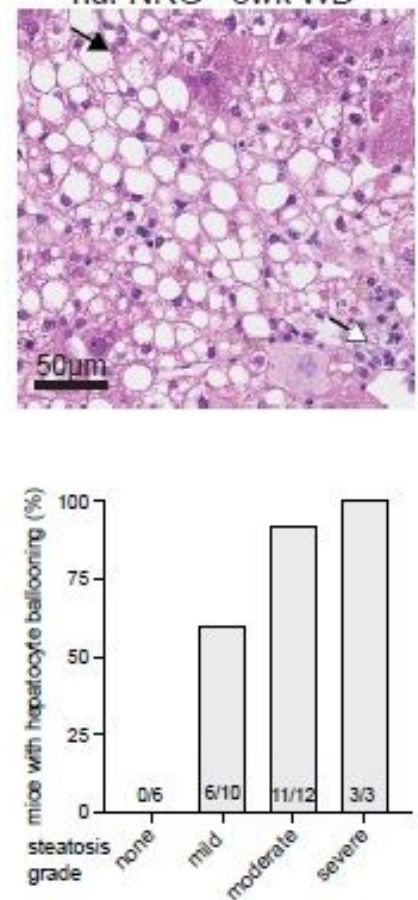

h

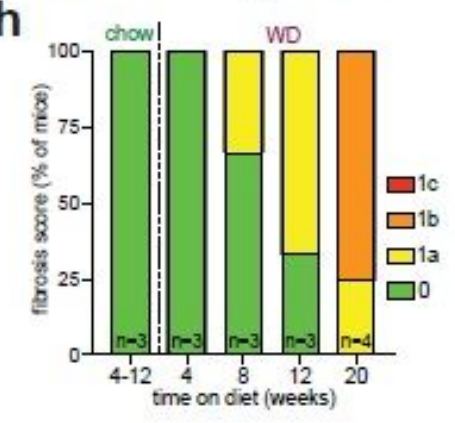

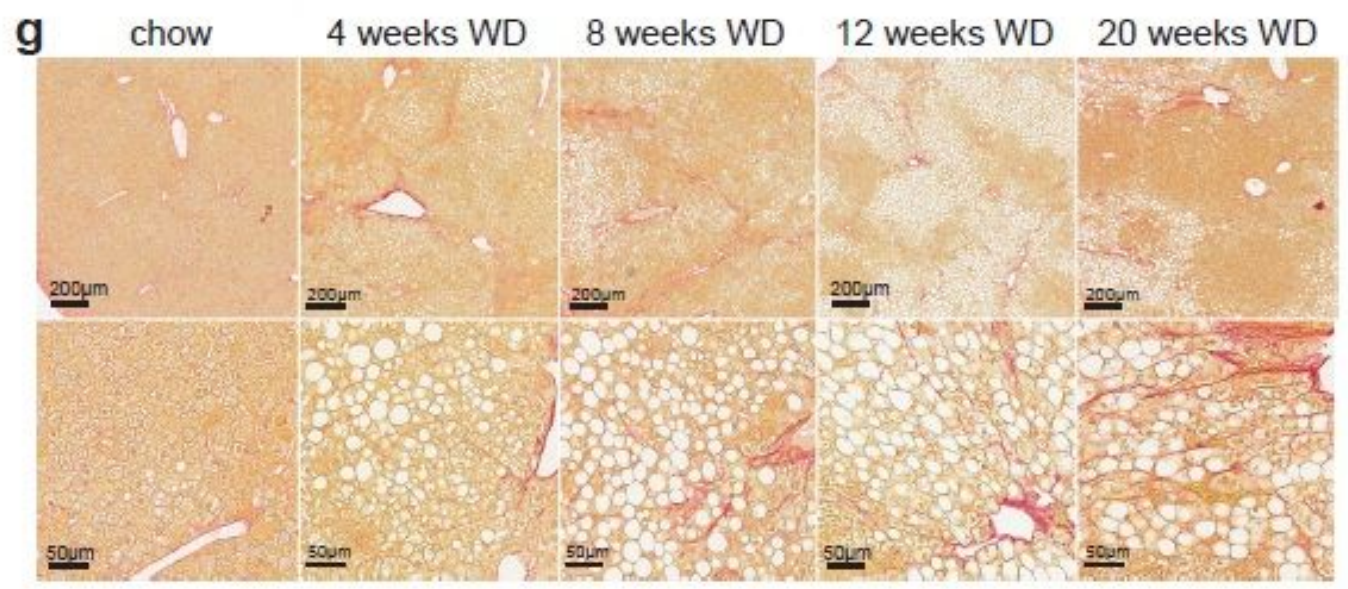

Figure 3

huFNRG mice on WD develop mild steatohepatitis a) ALT and AST activity in serum from huFNRG mice on chow and after 4 to 12 weeks on WD. Symbols individual mice, bars are median, unpaired t-test $\left.{ }^{* \star} \mathrm{p}<0.005,{ }^{* \star \star \star} \mathrm{p}<0.0001 . \mathrm{b}\right) \mathrm{H} \& \mathrm{E}$ staining from a huFNRG mouse liver after 8 weeks on WD. 734 Black arrow indicates hepatocyte ballooning degeneration and white arrow indicates lobular inflammation. c) Staining against human nuclear mitotic apparatus- 1 in the liver of a huFNRG mouse after 4 weeks on 
WD. Black arrow indicates hepatocyte ballooning. d) Fractions of huFNRG mice with human hepatocyte ballooning degeneration on chow and WD over time. Number of mice with ballooning per group at bottom of bars. e) Fractions of huFNRG mice with human hepatocyte ballooning degeneration by grade of steatosis. Number of mice with ballooning per group at bottom of bars. f) NAFLD activity score (NAS) in the human graft of huFNRG mice on chow and after 4 to 20 weeks on WD. Symbols individual mice, bars are median, unpaired t-test ${ }^{* \star} p<0.005,{ }^{* * *} p<0.0001$. g) Picosirius Red staining for collagen in livers from huFNRG mice on chow and after 4 to 20 weeks on WD. h) Fibrosis stages in the human graft of huFNRG liver on chow and after 4 to 20 weeks on WD. Mouse numbers at bottom of bars. 
a

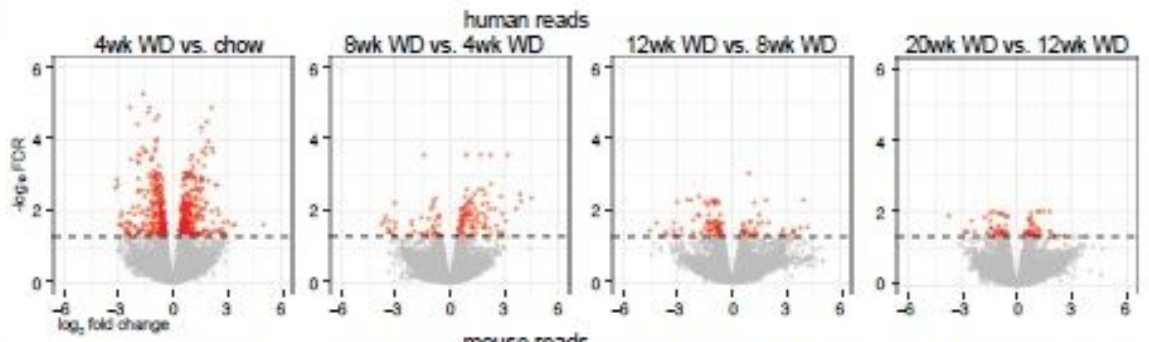

b

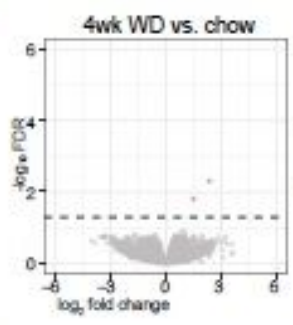

Bwk wD wouse reads

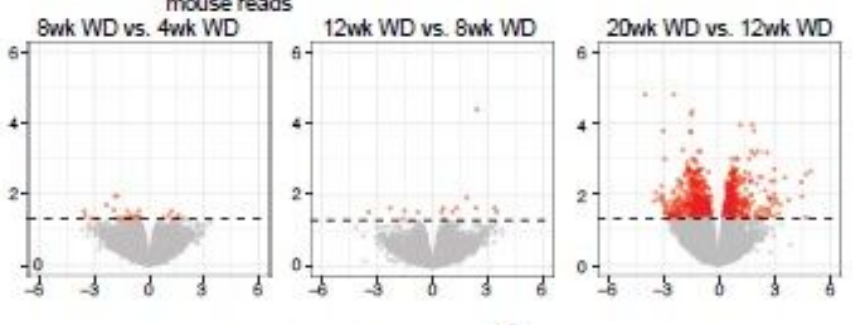

c

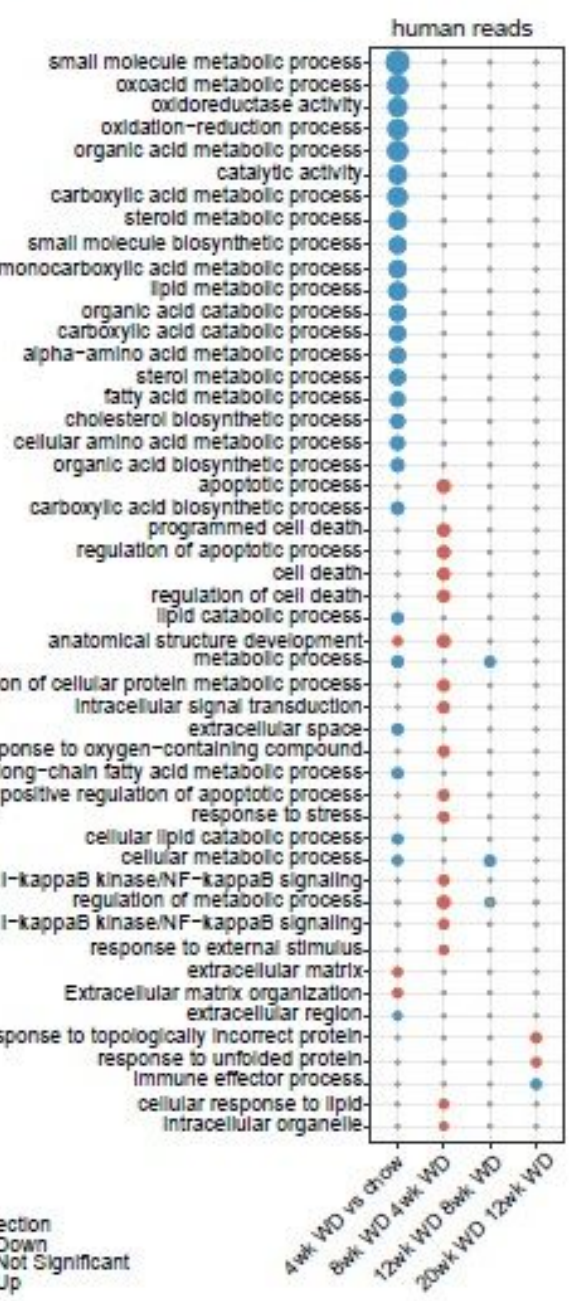

d

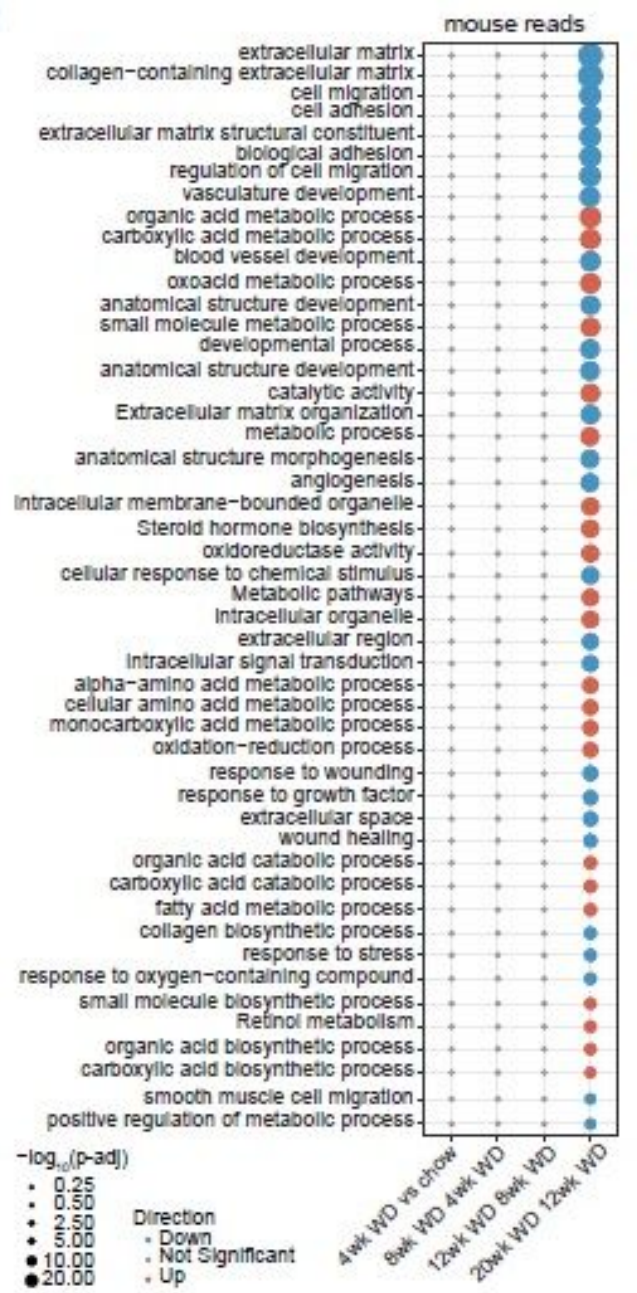

\section{Figure 4}

Transcriptional changes in huFNRG mice on WD a) Volcano plots of liver transcripts mapped to human genome (human reads) comparing huFNRG mice 4 weeks on WD to chow, and longer WD durations to the previous WD timepoint. RNA-sequencing of 3-4 mice per group, red symbols FDR $<0.05$, grey denotes not significant. b) Volcano plots of liver transcripts mapped to mouse genome (mouse reads) comparing huFNRG mice 4 weeks on WD to chow, and longer WD durations to the previous WD 
timepoint. RNA-sequencing of 3-4 mice per group, red symbols FDR $<0.05$, grey denotes not significant. c) Gene-ontology (GO) pathway analysis of human reads from livers of huFNRG mice 4 weeks on WD to chow, and longer WD durations to the previous WD timepoint. Blue symbols are down and red symbols are upregulated pathways, grey denotes no significant changes, symbol size indicates statistical significance. d) Gene-ontology (GO) pathway analysis of mouse reads from livers of huFNRG mice 4 weeks on WD to chow, and longer WD durations to the previous WD timepoint. Blue symbols are down and red symbols are upregulated pathways, grey denotes no significant changes, symbol size indicates statistical significance. 
a

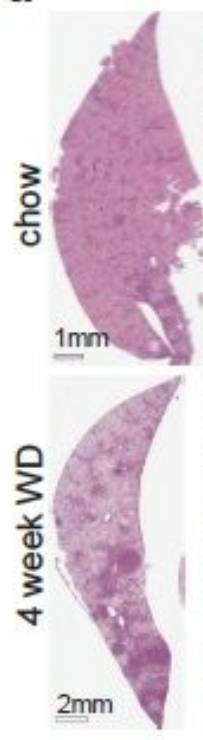

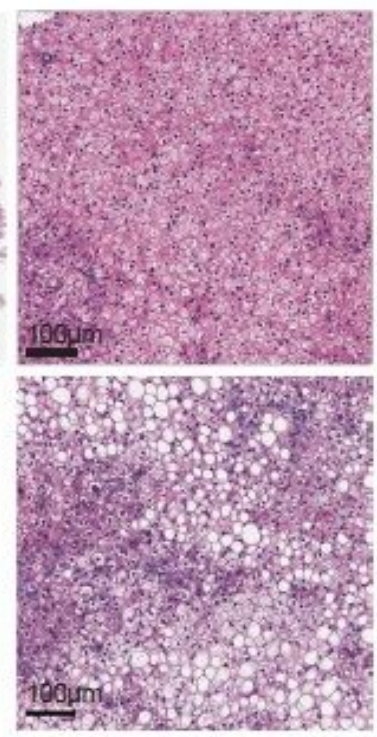

b

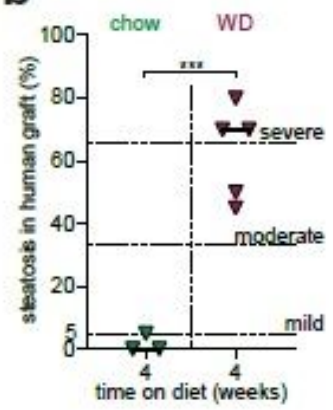

C

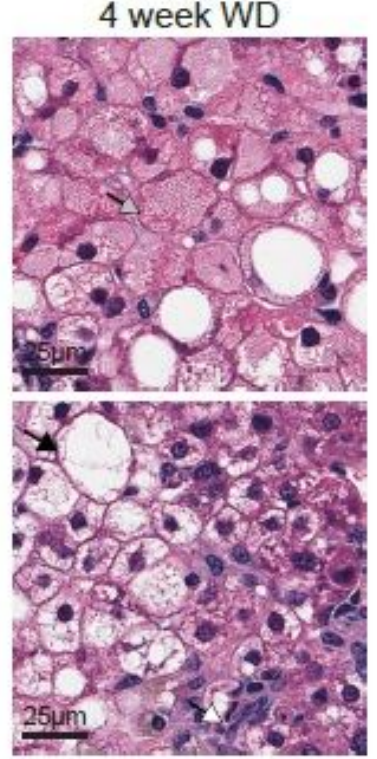

d

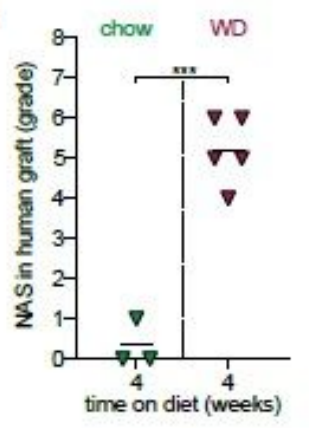

g $\Delta$ WD 148M-huFRG vs. $\triangle$ WD 1481-huFNRG

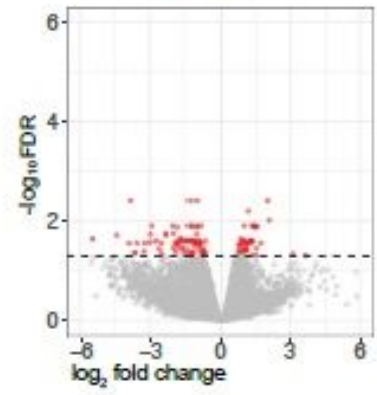

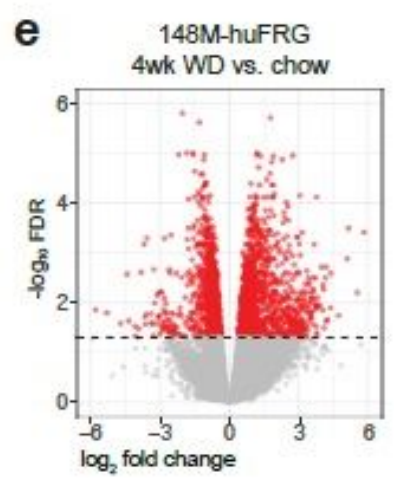

f

amall molecule metabolic process oxoacid metabolic proces: carboxylic acid metabolic process

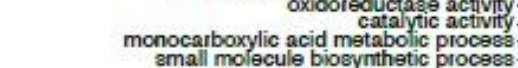
amall molecule biosynthetic process carboxy ic acid catap ic proces organic acid catabolic procers alpha-amino acid metabolic procasa (a) regulation of sterol biosynthetic process regulation of cholesterol biosynthetic process active organic acid biosynthetic procas: Activation of gene expleseion by SREBF (SREBP)
(SRER

Extracellular metabolic procas: lipid catacellular matrix
extracelich process long-chain fatty acid metabo ic proces: cellular metabolic process mitochondrial membrane.
mitochondrial mattix. election tranaport chain oxidative phosphorylation espiratory electron tranaport chain ATP synthesis coupled election transport.

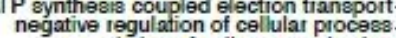
regulation of cell communication regulation of signal transduction regulation of hydrolase activity proteasome-mediated ubiquitin-dependent protein catabolic process. acetyl-CaA metabolic process fatty-acyl-CoA binding
fatty acid beta-oxidation. fatty acid alpha-oxidation. $-\log$ (value) 0.25 $: 0.50$ $: 2.50$ - 20.00

Direction

- Nown Significant

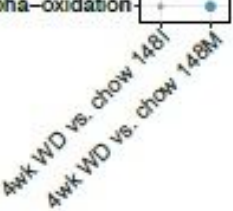

\section{Figure 5}

PNPLA3-148M huFRG mice on WD develop steatohepatitis a) H\&E staining on livers from 148M-huFRG mice on chow and after 4 weeks on WD. b) Clinical steatosis score of the human graft on livers from 148M-huFNRG mice on chow and after 4 weeks WD. Symbols are individual mice, bars median, unpaired t-test $* * * p<0.0005$. c) H\&E staining on livers from 148M-huFRG mice 4 weeks on WD. Grey arrow indicates Mallory Denk body, black arrow ballooning degeneration and white arrow lobular inflammation. d) NAFLD 
Activity Score (NAS) in the human graft of 148M-huFRG mice 807 on chow and after 4 weeks WD. Symbols individual mice, bars are median, unpaired t-test $\star \star \star p<0.0005$. e) Volcano plots of liver transcripts mapped to human genome (human reads) comparing 148M huFRG mice after 4 weeks WD vs. chow. RNA-sequencing of 3-4 mice per group, red symbols FDR $<0.05$, grey denotes not significant. $\mathrm{f}$ ) Gene-ontology (GO) pathway analysis of human reads comparing 148I-huFNRG and 148M huFRG livers after 4 weeks WD vs. chow. Blue symbols are down- and red symbols are upregulated pathways, grey denotes no significant changes, symbol size indicates statistical significance. g) Volcano plots of human reads differentially expressed after 4 weeks WD compared to chow $(\triangle W D)$ in 148M-huFRG livers versus 148 I-huFNRG livers. Red symbols FDR $<0.05$, grey denotes not significant. 
a

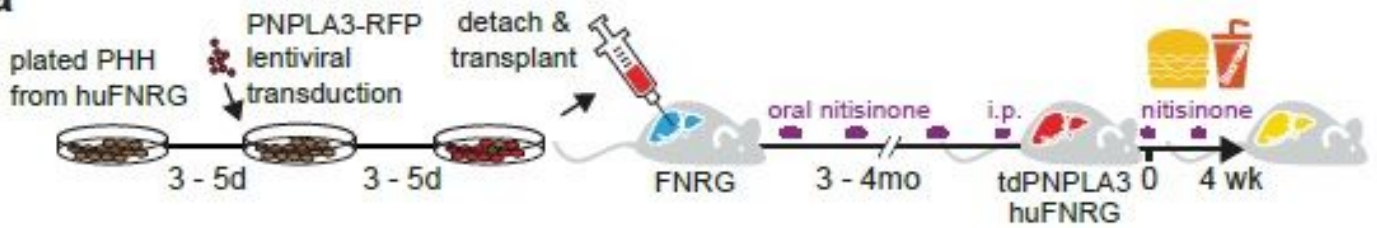

b tdRFP WD
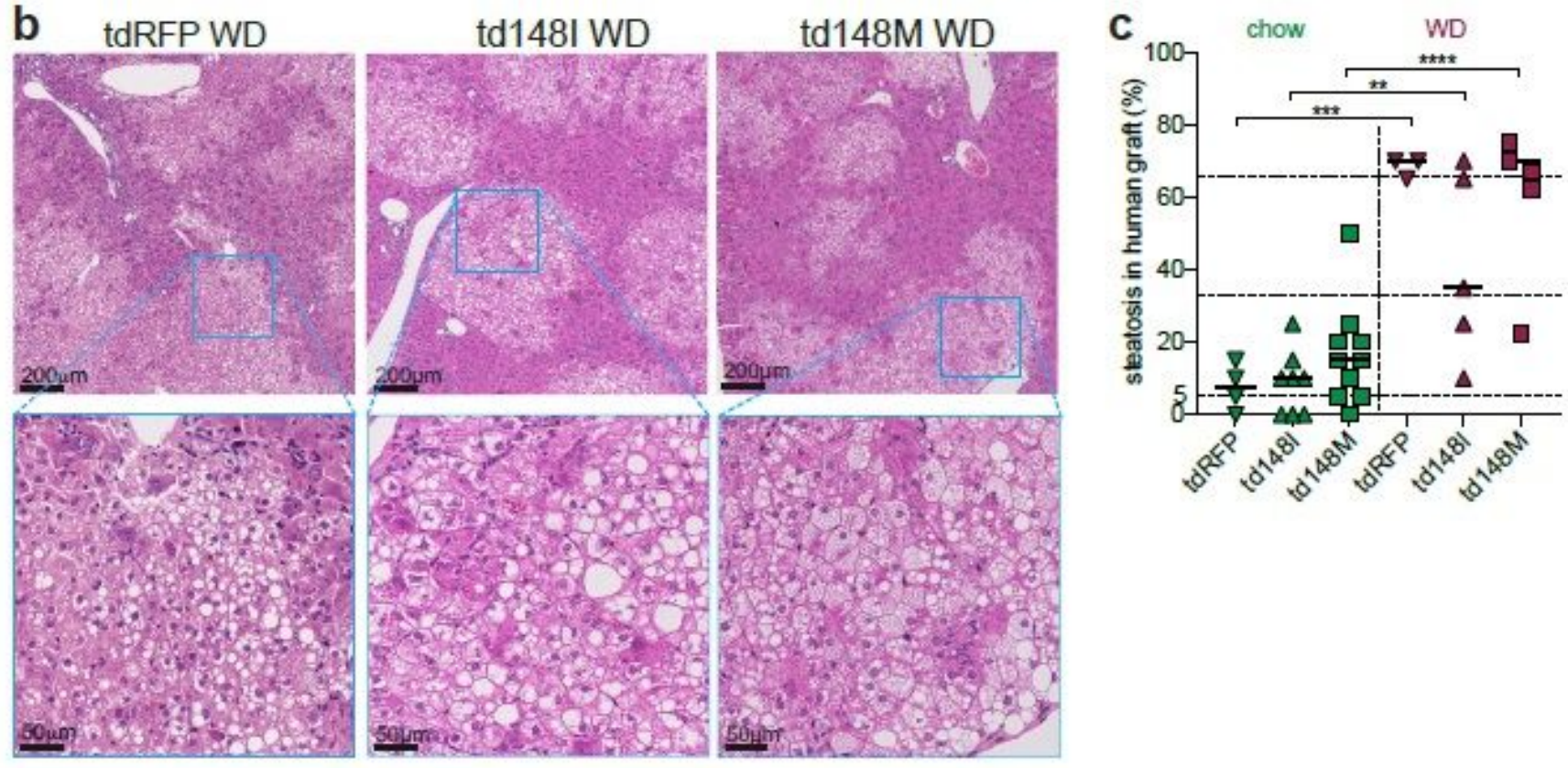

f

td 148 I vs. td $148 \mathrm{M}-\mathrm{WD}$
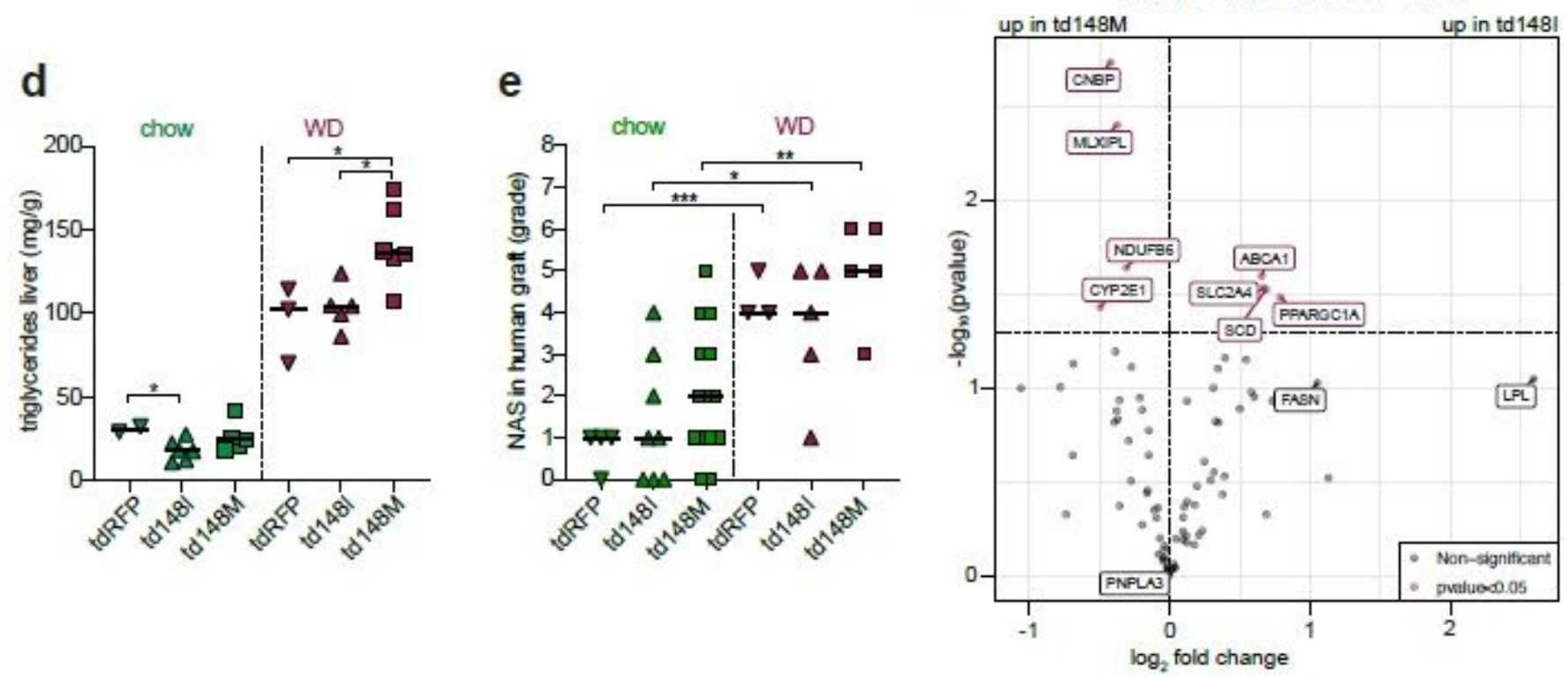

\section{Figure 6}

PNPLA3-148M overexpression in hepatocytes exacerbates steatosis in mice on WD a) Experimental timeline of creating PNPLA3 overexpressing mice and diet challenge. Mouse passaged primary human hepatocytes $(\mathrm{PHH})$ cultures were transduced with lentiviral vectors expressing PNPLA3 variants and retransplanted. Following humanization, huFNRG with PNPLA3 transduced hepatocytes (td) were exposed to 4 weeks of WD. b) H\&E staining on livers from tdRFP, td148I and td148M mice after 4 weeks on WD. c) 
Liver steatosis score of the human graft in tdRFP, td148I and td148M mice on chow and after 4 weeks on WD. Symbols individual mice, bars are median, unpaired t-test with ${ }^{\star *} p<0.005, * \star \star p<0.0005$ and $\star \star \star * x<0.0001$. d) Hepatic triglyceride quantification in tdRFP, td148I and td148M mice on chow and after 4 weeks on WD. Symbols individual mice, bars are median, unpaired t-test, ${ }^{*} \mathrm{p}<0.05$. e) NAFLD Activity Score (NAS) in the human graft of tdRFP, td148I and td148M mice on chow and after 4 weeks on WD. Symbols individual mice, bars are median, unpaired t-test, ${ }^{*} p<0.05,{ }^{* \star} p<0.005,{ }^{* \star} p<0.0005$.f) Volcano plot of 80 human genes expressed in livers of td148I versus td148M mice after 4 weeks on WD. Expression by qRT-PCR normalized to 11 housekeeping genes, $n=3-4$ mice per group, burgundy symbols $p<0.05$, grey symbols not statistically significant. 
a tdRFP HFD
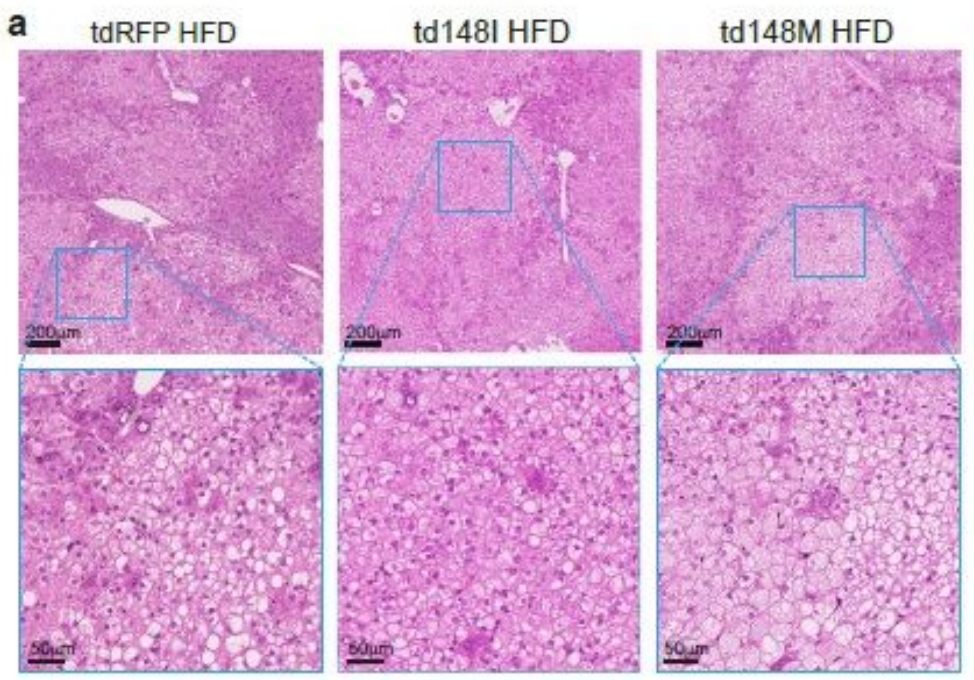

b

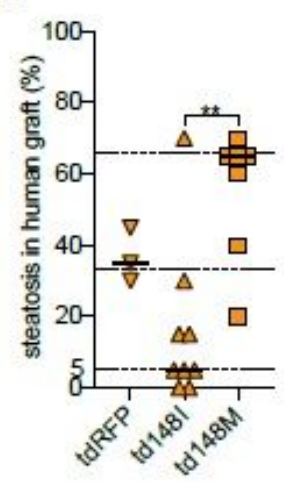

c td148M HFD

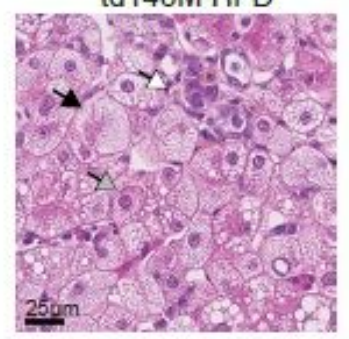

d

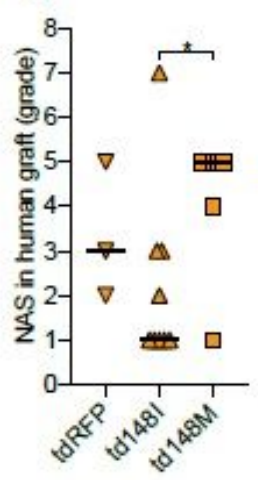

e

td148I HFD
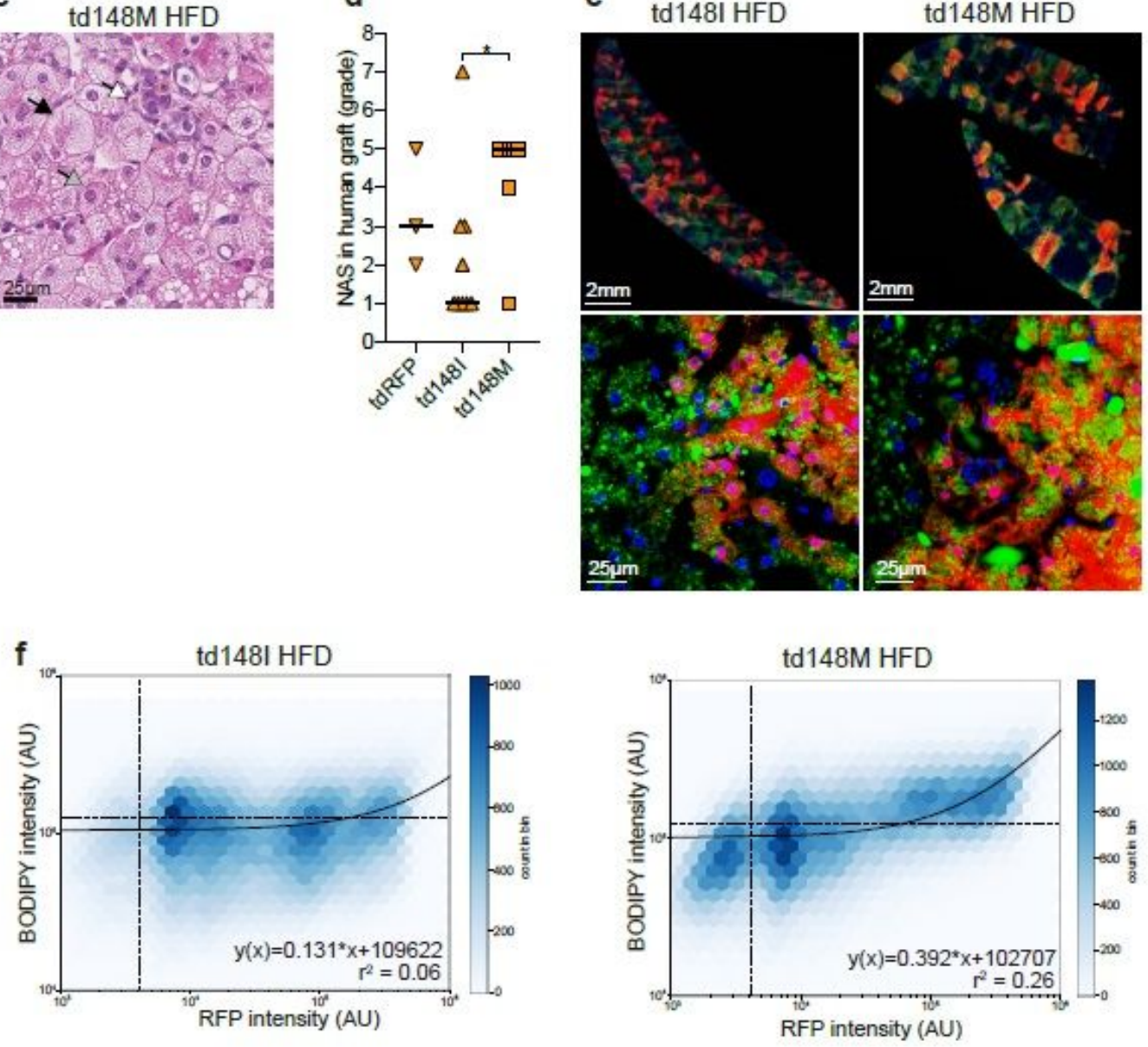

\section{Figure 7}

A HFD causes more active steatohepatitis in PNPLA3-148M overexpressing 881 micea) H\&E staining on livers from tdRFP, td148I and td148M mice after 4 weeks on HFD. b) Liver steatosis score of the human graft in tdRFP, td148I and td148M mice on chow and after 4 weeks on HFD. Symbols individual mice, bars are median, unpaired t-test with ${ }^{* \star} \mathrm{p}<0.005$. c) H\&E staining on liver from a td148M mouse after 4 weeks of HFD. White arrow indicates lobular inflammation, black arrow microvesicular steatosis and grey 
arrow Mallory-Denk body. d) NAFLD Activity Score (NAS) in the human graft of tdRFP, td148I and td148M mice on chow and after 4 weeks on HFD. Symbols individual mice, bars are median, unpaired t-test, ${ }^{*} p<0.05$. e) Low and high magnification fluorescent images of livers from td148I and td148M mice after 4 weeks of HFD stained for neutral lipids (green, BODIPY) and nuclei (blue, DAPI). f) Two-dimensional density plots of neutral lipids (BODIPY) and RFP of 355,161 small hepatocytes in livers from four td148I mice and 183,289 small hepatocytes in livers from three td148M mice after 4 weeks of HFD. Density plots are displaying RFP in X-, and BODIPY intensities in Y-Axis, both in arbitrary units (AU). Dashed lines display cell population group quadrants, with lower left quadrant harboring double negative and upper right quadrant harboring double positive cells. Solid line is simple linear regression with equation displayed underneath, $r 2$ is Pearson correlation coefficient. 